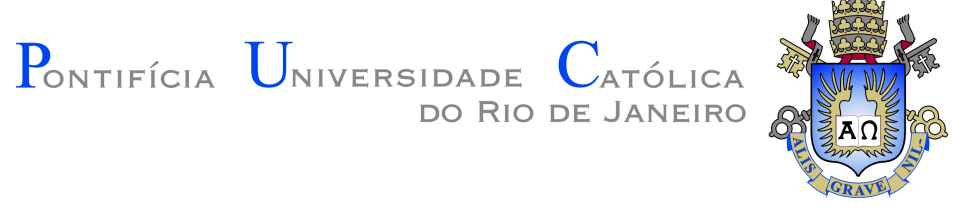

Ricardo Teixeira Leite

\title{
A novel and straightforward methodology to analyze materials under constant-structure oscillatory motion (SAOS and QL-LAOS)
}

Dissertation presented to the Programa de Pós-graduação em Engenharia Mecânica of PUC-Rio in partial fulfillment of the requirements for the degree of Mestre em Engenharia Mecânica.

Advisor: Prof. Paulo Roberto de Souza Mendes 


\title{
Pontifícia Universidade Católica $_{\text {a }}$

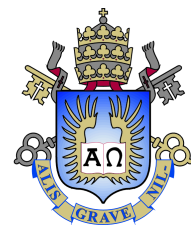

Ricardo Teixeira Leite

\section{A novel and straightforward methodology to analyze materials under constant-structure oscillatory motion (SAOS and QL-LAOS)}

Dissertation presented to the Programa de Pós-graduação em Engenharia Mecânica of PUC-Rio in partial fulfillment of the requirements for the degree of Mestre em Engenharia Mecânica. Approved by the undersigned Examination Committee.

\author{
Prof. Paulo Roberto de Souza Mendes \\ Advisor \\ Departamento de Engenharia Mecânica - PUC-Rio \\ Prof. Roney Leon Thompson \\ Programa de Engenharia Mecânica - COPPE - UFRJ \\ Dr. Flávio Henrique Marchesini de Oliveira \\ Departamento de Engenharia Mecânica - PUC-Rio \\ Prof. Márcio da Silveira Carvalho \\ Vice Dean of Graduate Studies \\ Centro Técnico Científico - PUC-Rio
}

Rio de Janeiro, September the 13th, 2017 
All rights reserved.

\section{Ricardo Teixeira Leite}

Ricardo Teixeira Leite graduated in Mechanical Engineering at Pontifical Catholic University of Rio de Janeiro (PUC- Rio) in 2015. Then, he started to work as a research engineer with rheology and non-Newtonian fluid mechanics in the Rheology Group (GReo) at PUC-Rio, where he was already involved in research activities for three years as an undergraduate student

Bibliographic data

Leite, Ricardo Teixeira

A novel and straightforward methodology to analyze materials under constant-structure oscillatory motion (SAOS and QL-LAOS) / Ricardo Teixeira Leite; advisor: Paulo Roberto de Souza Mendes. - Rio de janeiro: PUC-Rio , Departamento de Engenharia Mecânica, 2017.

v., 84 f: il. color. ; $30 \mathrm{~cm}$

Dissertação (mestrado) - Pontifícia Universidade Católica do Rio de Janeiro, Departamento de Engenharia Mecânica.

Inclui bibliografia

1. Engenharia Mecânica - Teses. 2. Reologia;. 3. Escoamento oscilatório;. 4. LAOS;. 5. QL-LAOS;. 6. Viscoelasticidade;. 7. Estrutura microscópica.. I. de Souza Mendes, Paulo Roberto. II. Pontifícia Universidade Católica do Rio de Janeiro. Departamento de Engenharia Mecânica. III. Título. 


\section{Acknowledgments}

I would like to first thank my advisor Paulo Roberto de Souza Mendes for our partnership along the last six years. I've learned a lot from you as an engineer and as a person. I would also like to thank Monica Nacacche for leading our research group alongside Paulo, always being a pleasent and important presence in our meetings. I'm also indebted to CNPq for providing me a scholarship and for the financial support to this research.

Then, I wish to thank all my fellow coworkers at the Rheology Group. Especially my "roomates" Aline Abdu, Bruna Leopércio, Carina Beline and Priscilla Varges. Thank you, Aline, for always listening to me whine during lunch and calming me down; Bruna for our beautiful friendship that preceds and will outlive our workmate relationship; Carina for the friday afternoons (listening to sertanejo out loud); and Priscilla for interrupting all of your (MANY) tasks to help me whenever I asked you a technical question (even though I didn't intend to have that much attention to the matter). I would also like to especially thank Eliana Marin for the incredible work on our parallel research with Petrobras and for holding the fort during the time I was focused on my thesis. I would also like to thank Flávio, for introducing me to research; and Alexandra, for giving me an everyday guidance throughout my graduation, making it possible for me to continue her research. At last, for your friendship, I would like to thank Pedro, Bruno, Elias, Behbood, PH, Roberta and Tati.

I believe true happiness is achieved through relationships, including the one you have with yourself. Hence, I am silently thanking every person that I eventually crossed paths with. Each of you helped me on this journey of becoming who I am today. However, I want to formally address some of those.

First, I would like to thank my family. You've been by my side ever since I was born and no one has given me more love and affection than you. My mom, Cristina, and my dad, Arnaldo, who've not only given me my genes but who've been by my side holding my hand everytime I needed. I couldn't ask for better parents. I am also extremely grateful to my grandparents Eduardo and Mara, for always bringing the family together and for their everlasting love. My cousin Ana Luiza, who was my first friend and who've come a long way by my side. I couldn't forget Toni, Ana Claudia, Bia, Laerte, Duda, Carol, Mario, Guido, Re, João, Monica, Jorge, Rafa, Luísa (who will officially join the family very soon), Isabela, Mathias, Darcy and Neyde. Lastly, I would like to thank Maria Zilda, my grandmother who I've never had the chance to meet, but to whom I am eternally grateful for bringing my father into the world and giving him so much love. 
Then, I would like to thank my friends. LE, for being my first friend out of my family and for coming all the way here by my side; together even life's the minor events (we are blue belts in Judo, after all). We may differ in many ways, but our essence is very similar. My other childhood friends, Gabriel, Max and LP. We've all taken different paths in life but whenever we are together it feels like as if we were kids again. Thanks, LE and Gabriel, for always inspiring me to try to be the best person I can. And thank you, Max and LP, for inspiring me to open myself to the world.

I am also grateful to my high school friends, who've sticked around even after all those years: Ana, Bernardo, Clarice, Cunha, Peixoto (the best student who ever graduated at CSI), Eric, Fábio, Guigas, Guilherme, Gustavo, Bel, Jo, Lu, Mari, Mia, Paula, Braço, Tai and Thomás. I also have to thank my friends from PUC, who've helped me through the suffering that is the engineering school: Adolpho, Alice, Sud, Lulu, Bruna, Bruna10, Trin, Eric, Johannes and Kevin; my friends from german class, who may remember only a few words in German but have never forgotten our friendship: Fernanda, Blumen (that's a word we won't forget) and Roberta; and my friends from Science without Borders, who were with me during the period of my life that, until today, feels like it was a dream: Adriano, Alvaro, Bola, Nugget, Cesinha, Fel, Fred, Rattes, Abdalla, Brugger, Jamal, Pedrinho, Ivo, Rangel and Raquel (who was by my side in many of the best moments of my life).

2017 is being a life-changing year for me. I would like to individually thank some of those who are being an active part of this process.

Ana, for being the angel on my shoulder. Thank you for being my reasoning. We teach a lot to each other and I am very proud of our friendship and our journey this year.

Alice, for being my best friend. For saying "yes" to "try different things". And for the partnership in every second of the last \#3months (7 years?).

Bit, for reminding me that the best people are still out there, waiting for me to meet them. For teaching me that deep connections may be made at a glance, or with a few words.

Fábio, for your smile and your heart. You show everyone the meaning of true friendship and purity of heart.

Lulu, for not giving up on going to Amigos da Onça, where it all started. For your high spirit and enthusiasm. For being the best storyteller ever and for making me laugh whenever we are together.

Thomás, thank you for the flow. We've walked a similar path this year and you always inspire me to be strong and make hard decisions. 
May the flow be with us all.

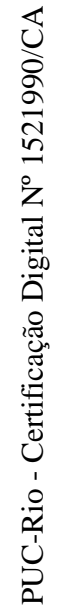




\section{Abstract}

Leite, Ricardo Teixeira; de Souza Mendes, Paulo Roberto (Advisor). A novel and straightforward methodology to analyze materials under constant-structure oscillatory motion (SAOS and QL-LAOS). Rio de Janeiro, 2017. 84p. Dissertação de Mestrado - Departamento de Engenharia Mecânica, Pontifícia Universidade Católica do Rio de Janeiro.

In this research, we developed a novel methodology to analyze materials in the linear and quasilinear oscillatory regimes (constant structure motions). It was shown that very few rheometric experiments are necessary. Furthermore, data analysis presented on this thesis is straightforward as raw data obtained from the rheometer requires simple data processing before being input into the equations that evaluate the material functions. This fact is in contrast with most large amplitude oscillatory shear analysis methods since they aim to analyze structure-changing motions and this requires complex mathematical manipulation of data. At last, both experimental methodology and data analysis presented in this research are much simpler than the previous methodologies used to analyze materials in the QL-LAOS regime.

\section{Keywords}

Rheology; Oscillatory flow; LAOS; QL-LAOS; Viscoelasticity; Microscopic structure. 


\section{Resumo}

Leite, Ricardo Teixeira; de Souza Mendes, Paulo Roberto. Metodologia simples e objetiva para análise de materiais nos regimes oscilatórios linear (SAOS) e quasilinear (QL-LAOS). Rio de Janeiro, 2017. 84p. Dissertação de Mestrado - Departamento de Engenharia Mecânica, Pontifícia Universidade Católica do Rio de Janeiro.

Nessa pesquisa, desenvolvemos uma nova metodologia para analizar materiais nos regimes oscilatórios linear e quasilinear. Foi mostrado que poucos experimentos reológicos são necessários. Além disso, a análise de dados apresentada é objetiva já apenas processamentos simples são necessários para avaliar as funções materiais. Esse fato contrasta com grande parte das análises de escoamento oscilatório de alta amplitude, tendo em vista que essas metodologias requerem manipulação matemática complexa dos dados. Por fim, a metodologia desenvolvida também apresenta grande evolução com relação às metodologias utilizadas anteriormente para analisar materiais no regime QL-LAOS.

\section{Palavras-chave}

Reologia; Escoamento oscilatório; LAOS; QL-LAOS; Viscoelasticidade; Estrutura microscópica. 


\section{Table of contents}

$\begin{array}{llr}1 & \text { Introduction } & 13\end{array}$

$\begin{array}{lll}1.1 & \text { Motivation } & 13\end{array}$

$\begin{array}{ll}1.2 \text { Research objectives } & 17\end{array}$

$\begin{array}{lll}1.3 \text { Outline } & 17\end{array}$

2 Background and literature $\quad 19$

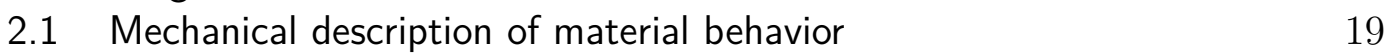

2.2 Viscoelasticity 21

$\begin{array}{ll}2.2 .1 & \text { Linear viscoelasticity } \\ 2.1 & 22\end{array}$

2.2.1.1 SAOStrain $\quad 22$

2.2.1.2 SAOStress $\quad 25$

2.3 Nonlinear viscoelasticity 28

2.3.1 Most used frameworks to analyze materials under LAOS 28

2.3.1.1 LAOStrain 35

$\begin{array}{ll}\text { 2.3.1.2 LAOStress } & 37\end{array}$

2.3.2 Quasilinear viscoelasticity 37

2.3.2.1 Framework used to analyse materials under QL-LAOS 38

2.4 Beyond the classical approaches 39

3 Experimental Set-Up and Methodology 44

3.1 Rheometric experiments 46

$\begin{array}{lll}3.2 & \text { Data processing } & 48\end{array}$

$4 \quad$ Results and discussion $\quad 52$

4.1 Flow Curve $\quad 52$

4.1.1 Gel $\quad 52$

4.1.2 Polyacrylamide solution 53

4.2 Stress Sweep $\quad 54$

4.2.1 Gel 54

$\begin{array}{lll}\text { 4.2.2 Polyacrylamide solution } & 57\end{array}$

5 Final remarks $\quad 61$

5.1 Future works $\quad 62$

$\begin{array}{lc}\text { Referências Bibliográficas } & 63\end{array}$

$\begin{array}{lll}\text { A Transient experiments } & 69\end{array}$

$\begin{array}{lll}\text { A.1 Creep } & 69\end{array}$

$\begin{array}{lll}\text { A.1.1 Kelvin-Voigt solid } & 70\end{array}$

$\begin{array}{ll}\text { A.1.1.1 Maxwell liquid } & 70\end{array}$

$\begin{array}{lll}\text { A.2 Stress relaxation } & 71\end{array}$

A.2.1 Kelvin-Voigt solid $\quad 72$

A.2.2 Maxwell fluid $\quad 72$

B The Jeffreys Mechanical Model $\quad 74$ 
C Inertia Correction

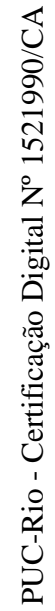




\section{List of figures}

Figure 2.1 Mechanical analogs for the Kelvin-Voigt (a) and Maxwell (b) mechanical models

Figure 2.2 Possible output stress waves (b) for a sinusoidal strain wave input (a)

Figure 2.3 Experimental Set-up and data analysis for the FTRheology analysis, extracted from (56)

Figure 2.4 The visual definition of the viscoelastic moduli in Lissajous plots, extracted from (12)

Figure 2.5 The visual representation of the SPP analysis, extracted from $(42)$

Figure 2.6 The Jeffreys mechanical analog.

Figure 3.1 The stress-controlled AR-G2 rheometer, manufactured by TA Instruments.

Figure 3.2 Details of the cross-hatched plate (a) and the aluminum plate (b).

Figure 3.3 Details of the sample placement method for the gel $((\mathrm{a}),(\mathrm{b}),(\mathrm{c}),(\mathrm{g}))$ and polyacrylamide solution $((\mathrm{d}),(\mathrm{e}),(\mathrm{f}),(\mathrm{g}))$.

Figure 3.4 Comparison between the analytical and experimental corrected stress.

Figure 4.1 Flow curve obtained with a cross hatched parallel-plates geometry, for the commercial hair gel.

Figure 4.2 Flow curve obtained with a smooth parallel-plates geometry, for the polyacrylamide dispersion.

Figure 4.3 Linearity map for a) the raw data, b) data after inertia correction, c) data after inhomogeneous flow correction and d) fully processed data

Figure 4.4 Jeffrey-based material functions for the commercial hair gel

Figure 4.5 Time constants for the commercial hair gel

Figure 4.6 Linearity map for a) the raw data, b) data after inertia correction, c) data after inhomogeneous flow correction and d) fully processed data

Figure 4.7 Dispersion of the storage modulus for distinct a) stress amplitudes and b) oscillatory regimes

Figure 4.8 Jeffrey-based material functions for the polyacrylamide solution

Figure 4.9 Time constants for the polyacrylamide solution

Figure A.1 Behavior of solid-like and fluid-like materials under a creep experiment

Figure A.2 Behavior of solid-like and fluid-like materials under a stress relaxation experiment 
Figure B.1 The Jeffreys mechanical analog. 


\section{Introduction}

\section{1}

\section{Motivation}

The macroscopic mechanical behavior of structured fluids, which is assessed by the measurement of rheological properties, is strongly related to their microscopic state. Macroscopic properties are related to the balance between externally imposed stresses tearing the microstructure apart and short-range attractive and Brownian forces building it up (41, 2, 50, 31, 46). This balance defines a microscopic state, commonly represented in the literature of structured fluids by a scalar parameter (frequently called structure parameter, denoted by $\lambda)(10,32,8)$.

Depending on the nature of the material, "microscopic state" may refer to fiber alignment, branching and stretching in polymers, emulsion droplet distribution, colloidal microstructure or gel network (41).

Rheometrical experiments usually produce macroscopic data, such as torque, displacement and normal force. However, a few macroscopic material properties that may be obtained through rheological experiments provide indirect and qualitative information about microscopic structural state (33). Other techniques such as light scattering (SANS, SALS and SAXS) and microscopy are necessary in order to obtain direct information about microscopic properties (22).

In shear rheology, two possible paths are usually followed. One possibility is to impose the shear stress to the sample, and then measure the kinematic response (shear and shear rate). The other type of experiment is the opposite, namely to control the shear (or shear rate) and then measure the dynamic response (shear stress). It is interesting to discuss about which approach is more consistent with the primary goal of rheology, namely to study the relationship between the applied load and the resulting deformation and flow of matter. Since changes in the shear stress are responsible for changes in the microscopic state, which in turn is directly related to the rheological properties, it is strategic in most applications to control the stress imposed to the material.

When the externally applied load is small in comparison with the 
Brownian forces, then the microscopic state remains the same as observed when the sample is in a quiescent condition (for a long time). In colloidal science, the usual parameter that compares the externally applied stress $\sigma$ with the Brownian stress $k_{B} T / a^{3}$ is the Péclet number (22):

$$
P e \equiv \frac{\sigma a^{3}}{k_{B} T}
$$

where $k_{B}$ is the Boltzmann constant, $T$ is the temperature, and $a$ is the particle radius. Therefore, the rheological properties measured under the condition of small Péclet number $(P e<<1$, often referred to as the linear viscoelastic regime) are independent of the applied stress. Examples of rheological measurements within the linear regime include (i) steady-state measurements at very small shear rates (and hence at very small stresses), which give the so-called zero-shear rate viscosity plateau; and (ii) small amplitude oscillatory shear (SAOS) measurements, which are obtained by applying a sinusoidal stress wave of small enough amplitude to ensure an undisturbed microscopic state during the oscillation cycles (7). The material functions obtained in SAOS measurements are the storage and loss moduli $G^{\prime}, G^{\prime \prime}$ and compliances $J^{\prime}, J^{\prime \prime}$, which do not depend on the stress amplitude.

However, in most industrial processes materials undergo high enough stresses to cause changes in the microscopic state. Examples of such processes include coating, spraying and injection molding (58). As a consequence, rheological properties measured in the linear regime are not sufficient to fully describe the material behavior, so that data pertaining to the nonlinear regime are necessary.

The steady-state flow experiment is a rheological experiment in the nonlinear regime that provides useful information (the flow curve and normal stresses) for industrial processes occurring at large timescales. The large amplitude oscillatory shear (LAOS) experiment is another rheological experiment in the nonlinear regime that aims at analyzing the transient response of materials, which is important in industrial processes involving high stresses and rapid changes.

Information about elasticity and characteristic times can only be obtained with transient flow experiments, and oscillatory experiments are recommended to obtain indirect information about the material microscopic state (33). Oscillatory experiments at large stress amplitudes are important to assess the macroscopic effects of changes in the microscopic state. This information is of course inaccessible via small amplitude oscillatory experiments, because in these experiments the microscopic state remains unchanged. 
Furthermore, when stresses are beyond the linear viscoelastic region, breakdown rate is a function of the stress level. At higher stresses, breakdown occurs at higher rates, while build up rates remain almost invariable. This leads to a shift in the equilibrium structural level, which also becomes a function of the stress $[\lambda(\sigma)]$. The rate of variation of the breakdown rate is also associated with the material's microscopic structure. Thus, complex materials with similar structures in the linear viscoelastic regime may present distinct structures and properties in the nonlinear regime (22).

The importance of nonlinear viscoelastic properties may be perceived in daily situations. Many studies attempt to correlate these properties to food characteristics such as composition and sensory attributes (52, 29, 30). Moreover, cosmetic industry has found that there is a good correlation between nonlinear rheological properties and sensory properties such as spreadability, slipperiness and tackiness (37). At last, researches in the bioengineering field have investigated injuries on biological tissues, such as human skin, under LAOS regime $(49,11)$.

However, difficulties arise when analyzing LAOS data. Since in this oscillatory flow the stress changes continuously along the cycle, then the microscopic state may also change continuously along the cycle. For example, a (non-thixotropic) soft solid under a LAOS test will present along the cycle a solid-like behavior at low stresses and a liquid-like behavior at high stresses. Consequently, when a large-amplitude sinusoidal stress wave is imposed, the strain or strain rate wave obtained is usually not sinusoidal. Conversely, when a large-amplitude sinusoidal strain wave is imposed, the stress wave obtained is usually not sinusoidal.

Complex strategies are needed in order to analyze this nonlinear response. The use of infinite Fourier series (FT-Rheology) to perform signal decomposition has been widely used $(25,16,55,24,17,18,23,57,58,60,21)$, following a common practice in many other fields of science to describe non-sinusoidal signals. On the other hand, the stress decomposition (SD) analysis (4) uses symmetry arguments and different basis functions to decompose the nonlinear stress response wave into elastic $\sigma^{\prime}$ and viscous $\sigma^{\prime \prime}$ components. This decomposition is elementary in the linear regime but non-unique in the nonlinear regime. Chebyshev polynomials of first kind $(12,13)$ were also chosen as the set of basis functions, since they generate an orthogonal space and thus isolate the elastic and viscous effects. These analyses are essentially mathematical descriptions of the nonlinear response waves, and have been criticized due to the complexity of the formulation and the lack of physical interpretation $(4,45,42,47,19,26,4,44,43)$. 
There are approaches in the LAOS literature to analyze non-sinusoidal response waves that attempt to incorporate the flow mechanics in a more sensitive way. Among them, the sequence of physical processes (SPP) (45) should be emphasized. This analysis describes the nonlinear response by considering two material functions that may be interpreted as generalized dynamic moduli, since they reduce to the classical storage and loss moduli in the linear viscoelastic limit.

The deviation from sinusoidal of the output wave stems from the continuous change in microscopic state that occurs as a consequence of the continuous stress change along the cycle. At small amplitudes, these changes in the microscopic state are negligible. On the other hand, at large amplitudes, microscopic configuration is substantially changing during the cycle. A soft solid under LAOS regime, for instance, presents a solid-like behavior at low stresses and a liquid-like behavior at high stresses.

Since macroscopic and microscopic properties are intimately related to the microscopic structure, they are also being instantaneously modified; and so is kinematic response, which is the key information to evaluate the previously mentioned properties. Since many variables are being simultaneously varied during the experiment, data becomes difficult to analyze. Hence, the main weakness of the LAOS test precedes the analysis chosen to analyze the data.

Recently, the existence of a quasilinear LAOS regime at higher frequencies was indicated by predictions of a constitutive model for thixotropic elastoviscoplastic materials (5). In this sub-class of the LAOS regime, the output wave is sinusoidal - just like in the SAOS regime - because at high enough frequencies the cycle period is much shorter than the characteristic time for changes in microscopic state, which therefore remains constant along the cycle. The existence of the quasilinear LAOS regime was later confirmed by experimental observations with a commercial hair gel (6).

Therefore we can say that SAOS and QL-LAOS compose a class of constant-structure motions, while LAOS (at lower frequencies) is a class of structure-changing motions (6). However, it is important to emphasize that in the QL-LAOS regime, in contrast to what occurs in SAOS experiments (linear regime), the microscopic state is not the one found in the quiescent condition. Each imposed stress amplitude defines a specific microscopic state. Even though the SAOS and QL-LAOS analyses are exactly the same (because in both cases the microscopic state is constant and hence the output wave is sinusoidal), the QL-LAOS material properties depend both on the frequency $\omega$ and on the stress amplitude $\sigma_{a}\left(J^{\prime}\left(\omega, \sigma_{a}\right), J^{\prime \prime}\left(\omega, \sigma_{a}\right)\right.$ or $\left.G^{\prime}\left(\omega, \sigma_{a}\right), G^{\prime \prime}\left(\omega, \sigma_{a}\right)\right)$, while the SAOS material properties depend on the frequency only $\left(J^{\prime}(\omega), J^{\prime \prime}(\omega)\right.$ 
or $\left.G^{\prime}(\omega), G^{\prime \prime}(\omega)\right)$. Therefore, the QL-LAOS experiments allow the study of the material's viscoelastic properties as a function of its microscopic state, while SAOS experiments allow the same study, but for the microscopic state corresponding to the quiescent condition $(P e<<1)$ only.

A methodology to analyze data under constant-structure motion has been previously presented by de Souza Mendes et al. (6). The analysis of intra-cycle transient data and waveshapes was vital in order to prove the existence of the quasilinear regime and to expose the physical phenomena that leads materials to this behavior. However, the exposed methodology involves a complex analysis that requires a large amount of data and a high number of rheometric experiments.

Ultimately, as will be shown in chapter 2, the most valuable information obtained from this analysis is the model based material functions, which do not require intra-cycle transient data and waveshape analysis (51). Therefore, it is interesting to pursue a more efficient methodology in order to turn constantstructure motion analysis into a more feasible rheological analysis.

\section{2 \\ Research objectives}

The main objective of this research is to develop a new experimental methodology to characterize materials in constant-structure motions. The main goals are to lower the number of rheometric experiments and simplify data analysis.

\section{3 \\ Outline}

This thesis is divided into four more chapters besides this first one. In Chapter 2, the concept of viscoelasticity is elucidated and constitutive equations for different classes of materials are presented. It is shown that there is a constitutive model behind every framework, even though they are usually implicit. The constitutive models are revealed while we explain the oscillatory and transient (in Appendix A) rheological experiments. Still in Chapter 2, the detection of the QL-LAOS region is documented and LAOS experiments are criticized. At last, an analysis framework based the conversion of model parameters into material functions is presented.

Following, Chapter 3 outlines the methodology developed during the research. A description of the experimental set-up and list of the materials used in the experiments is presented, as well as the description of the necessary data processing. 
Chapter 4 presents the results of the rheological experiments. The previously mentioned data processing operations are performed and experimental data is used in the evaluation of the model-based material functions for a range of stress amplitudes and frequencies.

Finally, Chapter 5 brings the main conclusions of the present research. 


\section{2}

\section{Background and literature}

\section{1}

\section{Mechanical description of material behavior}

Rheology is the study of flow and deformation of matter under applied forces. It comprises every class of mechanical behaviors, ranging from Hookean solids to Newtonian fluids.

Hooke's law for shear stresses is given as

$$
\tau=G \gamma
$$

From the analysis of equation 2-1, it may be observed that the stress applied to the material causes immediate deformation. This deformation is related to the applied stress by a proportionality constant $G$, called shear elastic modulus. Furthermore, when the stress is removed, the material instantaneously returns to its initial undeformed state. Materials that behave according to the Hooke's law are called elastic solids.

On the other hand, Newton's law of viscosity is a constitutive equation that obey the following equation

$$
\tau=\mu \dot{\gamma}
$$

Therefore, the applied stress causes the material to deform at a constant rate, which is proportional to the stress magnitude and to the Newtonian viscosity $\mu$, which is a constant value for a given temperature. Materials that behave according to the Newton's law of viscosity are called viscous fluids.

It is vital to state that these are constitutive equations, not fundamental laws of nature. Therefore, they are approximations that hold in some materials and fail in others. Due to this constraint, these proportionality constants are called material functions.

Mechanical analogs are used to aid the understanding of the behavior of these materials. Hookean solids may be modeled as springs, since both comply to the same qualitative behavior. An external force imposed to a spring causes an immediate deformation $x$, proportional to the spring constant $K$, as shown in equation 2-3. 


$$
F=K x
$$

Meanwhile, Newtonian fluids may be modeled as dashpots. An external force applied to a dashpot causes a deformation at constant rate $\dot{x}$, proportional to the dashpot constant $c$, as shown in equation 2-4.

$$
F=c \dot{x}
$$

However, lots of materials have complex microscopic structure and presents mechanical behaviors that are qualitatively distinct from the ones presented above. As an example, some materials may present time dependent properties, while others may present shear dependent properties. In a simplistic point of view, this means that the material functions deviate from the constant behavior previously mentioned (i.e. $G=G(\gamma, t)$ and $\mu=\eta(\dot{\gamma}, t)$ ).

A wide class of complex materials is called viscoelastic, since they may be approximated by a combination of elastic and viscous effects. The mechanical behavior of these materials may also be represented by mechanical analogs that combine elastic (spring) and viscous (dashpot) effects. The most famous frameworks are the Kelvin-Voigt and Maxwell models, presented on figures 2.1(a) and 2.1(b) respectively.

(a)

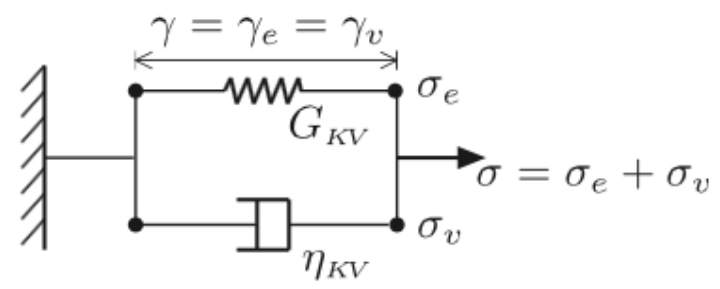

(b)

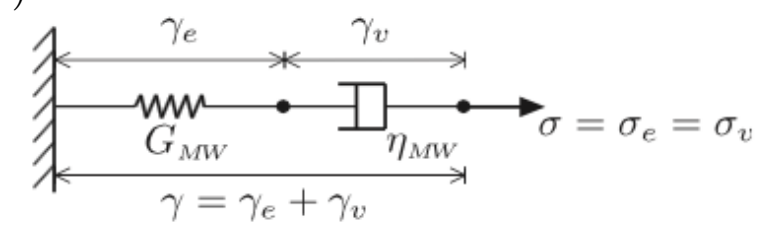

Figure 2.1: Mechanical analogs for the Kelvin-Voigt (a) and Maxwell (b) mechanical models

The Kelvin-Voigt model constitutive equation is given by

$$
\sigma=G_{K V} \gamma+\eta_{K V} \dot{\gamma}
$$

Meanwhile, the Maxwell model constitutive equation is given by

$$
\sigma+\frac{\eta_{M W}}{G_{M W}} \dot{\sigma}=\eta_{M W} \dot{\gamma}
$$


It is vital to state that the mechanical model employed in analysis frameworks is usually not explicit, nor are the consequences of its usage discussed. Thompson et al. (51) brought that discussion into light, proving that the Maxwell and the Kelvin-Voigt models are behind most oscillatory analysis in the literature. This discussion will be reproduced in sections 2.2.1.1, 2.2.1.2, 2.3.1.1 and 2.3.1.2.

Furthermore, the authors showed that these two models are not fit to describe some classes of mechanical behavior. Another mechanical model was proposed, along with a different analysis. These concepts will be addressed in section 2.4 .

\section{2}

\section{Viscoelasticity}

Viscoelastic materials are a class that comprises many materials, such as disks on human spine, biological tissues (i.e. skin), wood, concrete, polymers, among others. Their complex behavior usually comes from the fact that they possess complex microscopic structure and present a combination of elastic and viscous effects. Due to this combination, it is very interesting to study viscoelastic materials out of the steady-state regime, where this clash between elastic and viscous effects may be observed and indirect information about the material's microscopic structure may be obtained. There are two major classes of rheometric experiments usually performed on viscoelastic materials in order to analyze this combination of effects: dynamic and transient.

Dynamic experiments are performed either by imposing a sinusoidal deformation wave and measuring the corresponding mechanical behavior or by imposing a sinusoidal stress wave and measuring the material kinematic response. Even though it has already been addressed that imposing stress to the material is a more meaningful analysis from the physical phenomenon point of view, both approaches will be detailed. The former will be referred to as SAOStrain at small amplitudes and LAOStrain at large amplitudes, while the latter will be referred to as SAOStress at small amplitudes and LAOStress at large amplitudes. The definition of small and large amplitudes was already mentioned in section 1.1 but will be further described in future sections.

On the other hand, transient experiments involve applying a constant stress or deformation to the material and following its response before reaching the steady-state. Transient experiments may be branched into two types: creep and stress-relaxation. The former consists in applying a constant load to the material and noting the change of deformation with time. The latter measures the force required to maintain the deformation at a constant value. This value 
tends to fade with time, due to microscopic and molecular rearrangement. Rotation and relative flow between molecules, which governs macroscopic deformation, is time dependent since the breakage of the interparticle links is not instantaneous.

As it will be further addressed in sections A.1 and A.2, the characteristic times of these processes are called retardation and relaxation times, respectively. These time constants are very useful in rheological analysis since they are intimately linked to the microscopic structure of the material, thus providing valuable information about it.

\subsection{1}

\section{Linear viscoelasticity}

Currently, the most accepted definition of linear viscoelasticity is the regime at small amplitudes in which stress varies linearly with strain and shear rate. Also, material properties are independent of stress, strain and shear rate amplitudes throughout the entire regime.

As it was previously mentioned in section 1.1, the balance between stresses pulling structures apart and short-range attractive forces building the structure up through particle collision defines the material structural arrangement. Amplitudes in the linear viscoelastic regime are so small that the structure does not suffer significant modifications.

Linear viscoelasticity has been extensively studied throughout many decades and currently has a well-developed framework, which is relevant to the development of the theories exposed in the subsequent sections.

\subsubsection{1}

\section{SAOStrain}

SAOStrain are strain-controlled experiments in the linear viscoelastic region. As it was previously mentioned, the deformation input wave has the following form

$$
\gamma(t)=\gamma_{a} \sin (\omega t)
$$

where $\gamma_{a}$ is the deformation amplitude and $\omega$ is the oscillation frequency. This input wave may also be presented in terms of shear rate:

$$
\dot{\gamma}(t)=\gamma_{a} \omega \cos (\omega t) \rightarrow \dot{\gamma}(t)=\dot{\gamma}_{a} \cos (\omega t)
$$

At small amplitudes, the corresponding stress wave will also be a sinusoidal signal, of the same frequency.

$$
\sigma(t)=\sigma_{a} \sin (\omega t+\delta)
$$


where $\delta$ represents the phase shift between the strain and the stress waves.

This phase shift accounts for the viscoelastic effects acting on the material. The stress in a purely elastic (Hookean) solid is directly proportional to the imposed strain, thus it should be in phase with the sinusoidal strain wave described in equation 2-7. Meanwhile, the stress in a purely viscous fluid (Newtonian) is directly proportional to the shear rate. Thus, it should be in phase with the cosine wave described in equation 2-8.

Since $\delta$ is related to the sinusoidal signal, a purely elastic solid has a phase shift $\delta=0^{\circ}$, a purely viscous fluid has a phase shift $\delta=90^{\circ}$ and any other viscoelastic material has an intermediate phase shift. This behavior is better depicted below, in figure 2.2 .

(a)

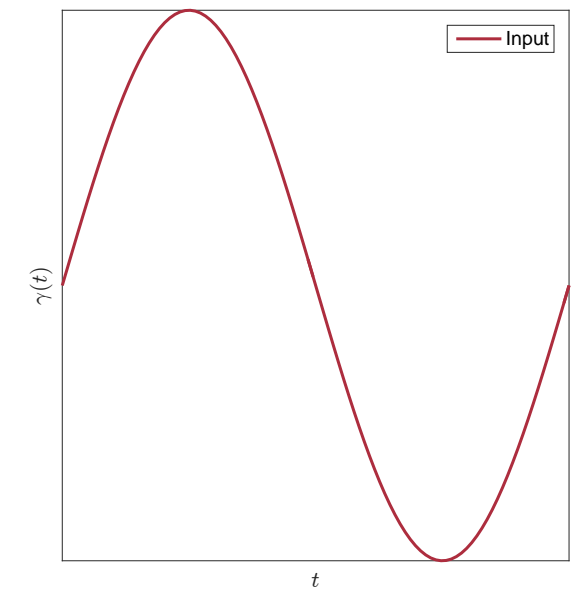

(b)

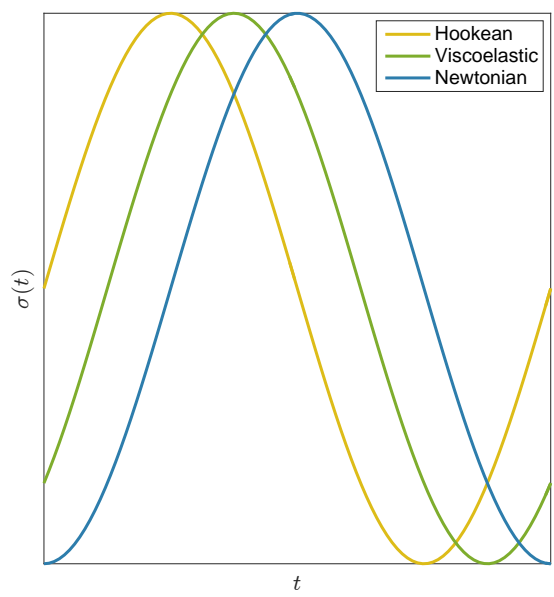

Figure 2.2: Possible output stress waves (b) for a sinusoidal strain wave input (a)

The stress signal presented on equation 2-9 may be decomposed in two waves of the same frequency: one in phase with the strain wave $(\sin (\omega t))$ and the other in phase with the shear rate wave $(\cos (\omega t))$; thus, $90^{\circ}$ out of phase with the strain wave.

$$
\sigma(t)=\sigma_{a} \cos (\delta) \sin (\omega t)+\sigma_{a} \sin (\delta) \cos (\omega t)
$$

As well as $G$ and $\mu$ were defined to form the constitutive equations 2-1 and 2-2, respectively; two material functions may be defined from equation 2-10 to form a constitutive equation for SAOStrain

$$
G^{\prime}(\omega)=\frac{\sigma_{a}}{\gamma_{a}} \cos (\delta), \quad G^{\prime \prime}(\omega)=\frac{\sigma_{a}}{\gamma_{a}} \sin (\delta)
$$

where $G^{\prime}$ is the elastic or storage modulus and $G^{\prime \prime}$ is the viscous or loss modulus. Thus, equation 2-10 may be rewritten as 


$$
\sigma(t)=G^{\prime}(\omega) \gamma_{a} \sin (\omega t)+G^{\prime \prime}(\omega) \gamma_{a} \cos (\omega t)
$$

Applying equations 2-7 and 2-8 in equation 2-12, we obtain

$$
\sigma(t)=G^{\prime}(\omega) \gamma(t)+\frac{G^{\prime \prime}(\omega)}{\omega} \dot{\gamma}(t)
$$

It is evident that the elastic modulus is in phase with the strain wave component and the storage modulus is in phase with the shear rate wave component; which are ruled by elastic and viscous effects, respectively.

The comparison between equations 2-5 and 2-13 shows that the framework behind the SAOStrain analysis is the Kelvin-Voigt model. This is intuitive, since the Kelvin-Voigt model is composed by an elastic branch, subjected to an elastic stress $\sigma_{e}$ and a viscous branch, subjected to a viscous stress $\sigma_{v}$. The total stress applied to the material is the sum of these two components, so that $\sigma=\sigma_{e}+\sigma_{v}$.

The elastic stress is related to the Kelvin-Voigt shear modulus $G_{K V}$ as follows

$$
\sigma_{e}=G_{K V} \gamma
$$

Meanwhile, the viscous stress is related to the Kelvin-Voigt viscosity $\eta_{K V}$ as follows

$$
\sigma_{v}=\eta_{K V} \dot{\gamma}
$$

Hence, the Kelvin-Voigt model parameters may be related to the classic moduli

$$
G_{K V}=G^{\prime}(\omega) \quad \eta_{K V}=\frac{G^{\prime \prime}(\omega)}{\omega} \equiv \eta^{\prime}(\omega)
$$

And material functions may be created using the Kelvin-Voigt model parameters as basis

$$
G_{K V}^{M F}=G_{K V}=G^{\prime}(\omega) \quad \eta_{K V}^{M F}=\eta_{K V}=\frac{G^{\prime \prime}(\omega)}{\omega} \equiv \eta^{\prime}(\omega)
$$

being $G_{K V}^{M F}$ the shear modulus material function, $G_{K V}$ the shear modulus of the Kelvin-Voigt model, $\eta_{K V}^{M F}$ the viscosity material function and $\eta_{K V}$ the Kelvin-Voigt model viscosity.

For a perfect Kelvin-Voigt solid, SAOStrain experiments will yield constant values for $G^{\prime}$ and $\eta^{\prime}$, thus $G_{K V}^{M F}$ and $\eta_{K V}^{M F}$ will be constant and equal to $G_{K V}$ and $\eta_{K V}$.

When SAOStrain experiments yield frequency dependent $G_{K V}^{M F}(\omega)$ and $\eta_{K V}^{M F}(\omega)$, the mechanical behavior gets farther from the one predicted by the Kelvin-Voigt model. Hence, it may be stated that the frequency dependence 
of these material functions is a measure of deviation from the employed framework.

Small deviations are expected for some solids. However, liquids under SAOStrain behave in a qualitatively different manner, thus using this framework to analyze data of liquids under SAOStrain produces huge inconsistencies. A perfect Maxwell liquid, for example, will yield the following material functions when analyzed with the Kelvin-Voigt framework

$$
\begin{gathered}
G_{K V}^{M F}=G^{\prime}=\frac{\eta_{M W}^{2} \omega^{2}}{G_{M W}^{2}+\eta_{M W}^{2} \omega^{2}} \cdot G_{M W} \\
\eta_{K V}^{M F}=\eta^{\prime}=\frac{G_{M W}^{2}}{G_{M W}^{2}+\eta_{M W}^{2} \omega^{2}} \cdot \eta_{M W}
\end{gathered}
$$

The Kelvin-Voigt material functions become a more complex function of $\omega$. Furthermore, both Maxwell model parameters $G_{M W}$ and $\eta_{M W}$ appear in both expressions. The appearance of $\eta_{M W}$ in the expression for $G^{\prime}$ derives from the fact that $G^{\prime}$ is defined in terms of $\gamma$, which is an irrelevant quantity for liquids. Thus, it functions as a compensation to correct this qualitative difference of behaviors.

Again, it is vital to state that every framework is a representation of an ideal mechanical behavior and real materials are usually not perfectly represented by them. When the real material deviates qualitatively from the model framework, the material functions lose their physical meanings and the analysis becomes meaningless. However, small deviations from the ideal behavior are acceptable.

In this section, it was shown that the definition of the dynamic moduli $G^{\prime}$ and $G^{\prime \prime}$ is based on the assumption that the output wave is also sinusoidal, since they derive from the linear superposition of the in-phase and outof-phase components of the stress response wave. Moreover, it was proven that the Kelvin-Voigt framework is behind the SAOStrain experiments and, consequently, the dynamic moduli.

\subsubsection{2}

\section{SAOStress}

SAOStress are stress-controlled experiments in the linear viscoelastic region. The stress input wave is analogous to the deformation wave shown in equation 2-7, thus it is given as follows

$$
\sigma(t)=\sigma_{a} \sin (\omega t)
$$

hence 


$$
\dot{\sigma}(t)=\sigma_{a} \omega \cos (\omega t)
$$

and the deformation response wave is described as

$$
\gamma(t)=\gamma_{a} \sin (\omega t+\delta)
$$

thus

$$
\dot{\gamma}(t)=\dot{\gamma}_{a} \omega \cos (\omega t+\delta)
$$

The deformation wave may be decomposed in an in-phase and an outof-phase component, analogously to the stress wave decomposition employed in the previous section.

$$
\gamma(t)=\gamma_{a} \sin (\omega t) \cos (\delta)+\gamma_{a} \cos (\omega t) \sin (\delta)
$$

Again, two material functions may be defined from equation 2-23.

hence

$$
J^{\prime}(\omega)=\frac{\gamma_{a}}{\sigma_{a}} \cos (\delta), \quad J^{\prime \prime}(\omega)=\frac{\gamma_{a}}{\sigma_{a}} \sin (\delta)
$$

$$
\gamma(t)=J^{\prime}(\omega) \sigma_{a} \sin (\omega t)+J^{\prime \prime}(\omega) \sigma_{a} \cos (\omega t)
$$

Applying equations 2-19 and 2-20 to equation 2-25, the following constitutive equation may be obtained

$$
\gamma(t)=J^{\prime}(\omega) \sigma+\frac{J^{\prime \prime}(\omega)}{\omega} \dot{\sigma}
$$

Likewise, equation 2-22 may also be decomposed in an in-phase and an out-of-phase component.

$$
\dot{\gamma}(t)=\dot{\gamma}_{a} \sin (\omega t) \cos (\delta)+\dot{\gamma}_{a} \cos (\omega t) \sin (\delta)
$$

Likewise, two material functions may be defined to transform equation 2-27 into a constitutive equation.

hence

$$
\phi^{\prime}(\omega)=\frac{\dot{\gamma}_{a}}{\sigma_{a}} \cos (\delta), \quad \phi^{\prime \prime}(\omega)=\frac{\dot{\gamma}_{a}}{\sigma_{a}} \sin (\delta)
$$

$$
\dot{\gamma}(t)=\phi^{\prime}(\omega) \sigma_{a} \sin (\omega t)+\phi^{\prime \prime}(\omega) \sigma_{a} \cos (\omega t)
$$

Applying equations 2-19 and 2-20 to equation 2-29 we obtain

$$
\dot{\gamma}(t)=\phi^{\prime}(\omega) \sigma+\frac{\phi^{\prime \prime}(\omega)}{\omega} \dot{\sigma}
$$

Rearranging equation 2-30, we obtain

$$
\frac{\dot{\gamma}(t)}{\phi^{\prime}(\omega)}=\sigma+\frac{\phi^{\prime \prime}(\omega)}{\phi^{\prime}(\omega) \omega} \dot{\sigma}
$$

The comparison between equations 2-6 and 2-31 shows that the framework behind the SAOStress analysis is the Maxwell model. This is intuitive, 
since the Maxwell model is composed by a single branch, exposed to the same stress $\sigma$. The spring element is in series with the dashpot element. While the former is subjected to an elastic deformation $\gamma_{e}$ and responds immediately; the latter is subjected to a viscous deformation $\gamma_{v}$ and responds to its derivative $\dot{\gamma}_{v}$, which is out of phase with the stress signal.

The framework parameters $G_{M W}$ and $\eta_{M W}$ may be transformed in material functions that are related to the SAOStress material functions through

$$
G_{M W}^{M F}=\frac{\omega}{\phi^{\prime \prime}(\omega)} \equiv \frac{1}{J^{\prime}(\omega)} \quad \eta_{M W}^{M F}=\frac{1}{\phi^{\prime}(\omega)}
$$

For a perfect Maxwell liquid, SAOStress experiments will yield constant values for $1 / J^{\prime}$ and $1 / \phi^{\prime}$. Thus, the material functions $G_{M W}^{M F}$ and $\eta_{M W}^{M F}$ will be constant and equal to the equation's 2-6 proportionality constants $G_{M W}$ and $\eta_{M W}$, respectively.

When SAOStress experiments yield frequency dependent $G_{M W}^{M F}(\omega)$ and $\eta_{M W}^{M F}(\omega)$, the mechanical behavior gets farther from the one predicted by the Maxwell model. Hence, it may be stated that the frequency dependence of these material functions is a measure of deviation from the employed framework.

Analogously to our discussion for SAOStrain experiments, we may examine a perfect Kelvin-Voigt solid under a SAOStress experiment. The following material functions will be yielded

$$
\begin{aligned}
G_{M W}^{M F} & =\frac{1}{J^{\prime}}=\frac{\eta_{K V}^{2} \omega^{2}}{G_{K V}^{2}+\eta_{K V}^{2} \omega^{2}} \cdot G_{K V} \\
\eta_{M W}^{M F} & =\frac{1}{\phi^{\prime}}=\frac{G_{K V}^{2}}{G_{K V}^{2}+\eta_{K V}^{2} \omega^{2}} \cdot \eta_{K V}
\end{aligned}
$$

Once more, it is not straightforward to interpret $G_{M W}^{M F}$ and $\eta_{M W}^{M F}$ as $\omega$, $\eta_{K V}$ and $G_{K V}$ appear in both terms.

In this section, it was shown that the definition of the compliances $J^{\prime}$ and $J^{\prime \prime}$ as well as the definition of the fluidities $\phi^{\prime}$ and $\phi^{\prime \prime}$ are based on the assumption that the output wave is also sinusoidal, since they derive from the linear superposition of the in-phase and out-of-phase components of the deformation response wave. Moreover, it was proven that the Maxwell framework is behind the SAOStress experiments and, consequently, the aforementioned material functions. 


\section{Nonlinear viscoelasticity}

Unlike the linear viscoelastic regime, nonlinear viscoelasticity implies that amplitudes are high enough to cause changes in the material microscopic structure. At higher stresses, breakdown occurs at higher rates, while build up rates remain almost invariable. This leads to a shift in the equilibrium structural level, which also becomes a function of the stress $[\lambda(\sigma)]$.

Analogously to the linear viscoelastic regime, dynamic experiments in nonlinear viscoelastic regime are performed either by imposing a sinusoidal deformation wave and measuring the corresponding dynamic response or by imposing a sinusoidal stress wave and measuring the material kinematic response. However, the analysis frameworks for dynamic experiments at large amplitude oscillatory shear (LAOS) are not as well-established as the ones used in SAOS. Complications arise from the fact that, during an oscillation cycle, the instantaneous stress $\sigma(t)$ varies from small values, inside the linear viscoelastic region, to large values. Notice that $\sigma(t)$ sweeps an infinity of different stress values outside the linear viscoelastic region. Hence, it sweeps an infinity of microscopic structural states. As it was previously stated on chapter 1, each microscopic structure results in a different kinematic response for the input. Therefore, the output wave is not a sinusoidal wave in this regime.

The fact that the output wave is not sinusoidal implies that the linear signal decomposition used in sections 2.2.1.1 and 2.2.1.2 may not be employed in order to analyze the material. Hence, many frameworks have been presented as attempts to describe the nonlinear response waves. Section 2.3.1 will present some of the most used frameworks, as well as the critics on each of them. Meanwhile, sections 2.3.1.1 and 2.3.1.2 will show which frameworks describe LAOStrain experiments and which frameworks describe LAOStress experiments. At last, the mechanical models behind each approach will also be exposed.

\subsection{1}

\section{Most used frameworks to analyze materials under LAOS}

The first studies on LAOS started on the 50's (15), but hardware and software limitations, especially low torque transducer resolution and low computational power, severely hindered further progress at that time. More than half a century later, there is still a lot of room for discussion on the matter. Many methodologies to analyze materials under LAOS have been developed during this time and the most relevant ones are further discussed below.

The Fourier transform rheology was the first well established method for analyzing nonlinear viscoelastic oscillatory motions. FT-Rheology uses 
Fourier series to describe the nonlinear signal. The Fourier series consists in a sum of sinusoidal waves at harmonic frequencies, each one possessing distinct coefficients and phase angles. Equation 2-35 shows the Fourier series for a nonlinear stress response, while equation 2-36 shows the Fourier series for a nonlinear strain response.

$$
\begin{aligned}
& \sigma(t)=\sum_{n=1, o d d} \sigma_{n} \sin \left(n \omega t+\delta_{n}\right) \\
& \gamma(t)=\sum_{n=1, \text { odd }} \gamma_{n} \sin \left(n \omega t+\delta_{n}\right)
\end{aligned}
$$

In equation 2-35 the stress amplitude $\sigma_{n}\left(\omega, \gamma_{a}\right)$ and the phase angle $\delta_{n}\left(\omega, \sigma_{a}\right)$ of the harmonics depend on both frequency $\omega$ and strain amplitude $\gamma_{a}$. Meanwhile, in equation 2-36, the strain amplitude $\gamma_{n}\left(\omega, \sigma_{a}\right)$ and the phase angle $\delta_{n}\left(\omega, \sigma_{a}\right)$ depend on both frequency $\omega$ and stress amplitude $\sigma_{a}$. Notice that each wave has a frequency $n \omega$, meaning that it is the $n$ harmonic of the natural frequency $\omega$. Equations 2-35 and 2-36 may also be written as in-phase and out-of-phase terms, analogously to the forms presented for SAOStrain and SAOStress.

$$
\begin{aligned}
& \sigma(t)=\gamma_{a} \sum_{n=1, o d d}\left[J_{n}^{\prime}\left(\omega, \sigma_{a}\right) \sin (n \omega t)+J_{n}^{\prime \prime}\left(\omega, \sigma_{a}\right) \cos (n \omega t)\right] \\
& \gamma(t)=\sigma_{a} \sum_{n=1, o d d}\left[G_{n}^{\prime}\left(\omega, \sigma_{a}\right) \sin (n \omega t)+G_{n}^{\prime \prime}\left(\omega, \sigma_{a}\right) \cos (n \omega t)\right]
\end{aligned}
$$

where $G_{n}^{\prime}$ and $G_{n}^{\prime \prime}$ or $J_{n}^{\prime}$ and $J_{n}^{\prime \prime}$ are the Fourier coefficients. Since the wave is sinusoidal on the linear viscoelastic regime, the higher harmonics are negligible. Notice that all coefficients except $G_{1}^{\prime}$ and $G_{1}^{\prime \prime}$ or $J_{1}^{\prime}$ and $J_{1}^{\prime \prime}$ vanish in this case and the equation's coefficients reduce to the storage and loss compliances.

The ratios between the intensity of the higher-order harmonics and the intensity of the first-order harmonic are usually used as a measure of nonlinearity, especially the ratio between the third and the first harmonics $\left(I_{3} / I_{1}\right)$.

Wilhelm et al. (57, 58, 59, 55) developed the methodology for high sensitivity Fourier transform (FT) rheology by transferring techniques from NMR spectroscopy to oscillatory rheometery on commercial rheometers. Their work is distinguished by the development and use of extremely sensitive detection methods, obtaining high resolution torque signals from commercial rheometers (53). Experimentally, the measured information such as torque and displacement are Fourier transformed and further evaluated with a self- 
developed software, as illustrated in figure 2.3. The simple experimental setup was clearly beneficial for a broader adoption of the technique.
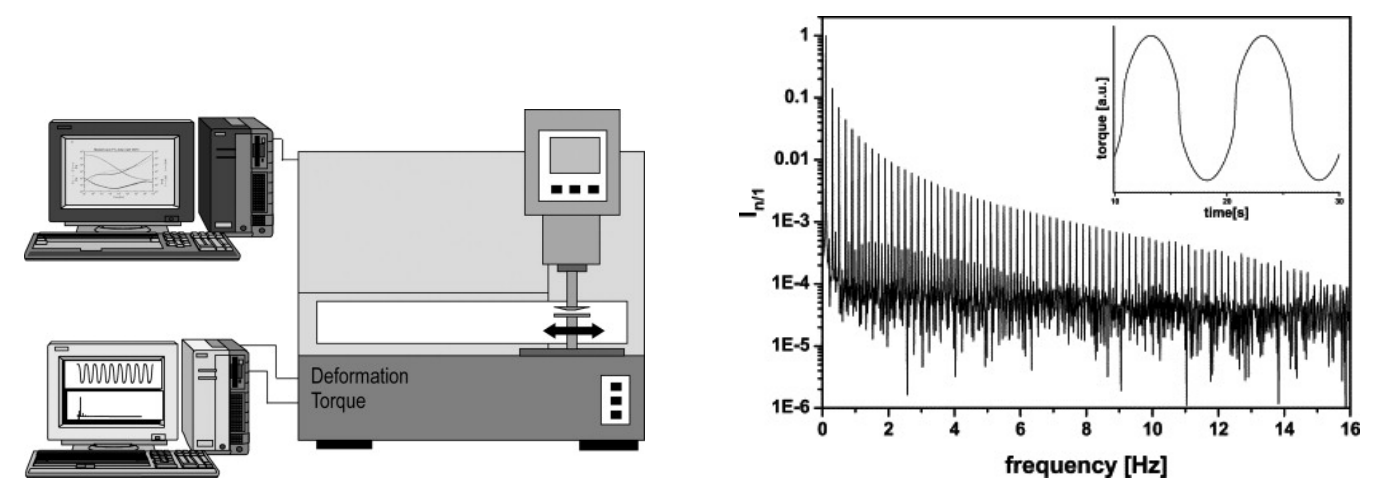

Figure 2.3: Experimental Set-up and data analysis for the FT-Rheology analysis, extracted from (56)

FT-Rheology was used for various complex fluids, such as polymer melts $(35,20,54)$, polymer solutions $(36,34)$, polymer blends (3), dispersed systems (55) and industrial elastomers $(28,27)$.

However, difficulties arise from the fact that experimental data set obtained in the time-domain $s(t)$ generates complex data in the frequency domain $s(\omega)$, with real and imaginary parts which are difficult to analyze and attribute physical meaning. Furthermore, it is also difficult to attribute physical meaning to the large number of coefficients $\left(G_{n}^{\prime}\right.$ and $G_{n}^{\prime \prime}$ or $J_{n}^{\prime}$ and $\left.J_{n}^{\prime \prime}\right)$.

In order to overcome these flaws in LAOS analysis, alternative techniques to quantify nonlinear behavior were developed. The decomposition of the nonlinear stress response wave into elastic $\sigma^{\prime}$ and viscous $\sigma^{\prime \prime}$ components was attempted. This decomposition is elementary in the linear regime, as it was shown in previous sections, however, unambiguously decompose the stress response wave in the nonlinear regime is a difficult task. According to Hyun (22), for this reason, "FT-Rheology does not decompose the nonlinear shear stress in terms of the deformation inputs themselves, but rather operates on the time-domain representation of the stress waveform, $\sigma(t)$, and quantifies nonlinear viscoelastic responses through intensity and phase angle of higher harmonics"

Using symmetry arguments, Cho et al. (4) managed to decompose the total stress into a superposition of elastic stress $\sigma^{\prime}(\gamma)$ and viscous stress $\sigma^{\prime \prime}(\dot{\gamma})$, defining stress as a function of independent inputs of strain and shear-rate $[\sigma=\sigma(\gamma, \dot{\gamma})]$ instead of a function represented in the time domain $(\sigma(t))$. The stress decomposition is given as follows 


$$
\sigma^{\prime}=\Gamma^{\prime}\left(x, \gamma_{a}\right) x, \quad \sigma^{\prime \prime}=\Gamma\left(y, \gamma_{a}\right) y
$$

where $\Gamma$ and $\Gamma$ are the generalized dynamic moduli, $x=\gamma$ and $y=\dot{\gamma}$. Cho et. al (4) suggested a polynomial regression of the form

$$
\begin{aligned}
\sigma^{\prime}\left(x, \gamma_{a}\right) & =G_{1}^{\prime}(\omega) x+G_{3}^{\prime}\left(\omega, \gamma_{a}\right) x^{3}+G_{5}^{\prime}\left(\omega, \gamma_{a}\right) x^{5}+\ldots+G_{n}^{\prime}\left(\omega, \gamma_{a}\right) x^{n} \\
\sigma^{\prime \prime}\left(x, \gamma_{a}\right) & =G_{1}^{\prime \prime}(\omega) x+G_{3}^{\prime \prime}\left(\omega, \gamma_{a}\right) x^{3}+G_{5}^{\prime \prime}\left(\omega, \gamma_{a}\right) x^{5}+\ldots+G_{n}^{\prime \prime}\left(\omega, \gamma_{a}\right) x^{n}
\end{aligned}
$$

However, the material properties obtained from this regression depend on the polynomial order, which is undesirable. Furthermore, $G_{1}^{\prime}$ and $G_{1}^{\prime \prime}$ do not correspond exactly to the classic linear viscoelastic moduli because the decomposition above suffer from non-orthogonality. Thus, Ewoldt $(13,12)$ proposed the use of Chebyshev Polynomials of the first kind to decompose the nonlinear stress wave. They represent a set of basis functions capable of describing the stress wave in the orthogonal space formed from the oscillation strain and the shear-rate. The use of an orthogonal basis simplifies the nonlinear response description as it no longer requires the consideration of the explicit temporal dependence, focusing, instead, on how the response varies with magnitude and rate of deformation. Using Chebyshev Polynomials, stress response is decomposed as

$$
\begin{aligned}
\sigma^{\prime}(x) & =\gamma_{a} \sum_{n: o d d} e_{n}\left(\omega, \gamma_{a}\right) T_{n}(x) \\
\sigma^{\prime \prime}(x) & =\dot{\gamma_{a}} \sum_{n: \text { odd }} v_{n}\left(\omega, \gamma_{a}\right) T_{n}(y)
\end{aligned}
$$

where $T_{n}(x)$ is the $\mathrm{n}$-th order Chebyshev polynomial of the first kind, $x=\gamma / \gamma_{a}, y=\dot{\gamma} / \dot{\gamma}_{a}, e_{n}\left(\omega, \gamma_{a}\right)$ the elastic Chebyshev coefficients, and $v_{n}\left(\omega, \gamma_{a}\right)$ the viscous Chebyshev coefficients. Ewoldt et al (12) also suggested viscoelastic moduli for the nonlinear regime: minimum-strain and large-strain elastic moduli; and minimum-rate and large-rate dynamic viscosities. These moduli are identified in the Lissajous plots presented in figure 2.4.

Lissajous curves are plots of parametric equations of sinusoidal form:

$$
\begin{gathered}
x(t)=a \sin (\omega t+\delta) \\
y(t)=b \sin (t)
\end{gathered}
$$

In rheological oscillatory experiments, Lissajous curves may be used to describe $\sigma(t) \times \gamma(t)$ or $\sigma(t) \times \dot{\gamma}(t)$ plots.

The minimum-strain modulus $\left(G_{M}^{\prime}\right)$ is the tangent modulus at zero instantaneous strain $(\gamma(t)=0)$, hence, at maximum $\dot{\gamma}$ 

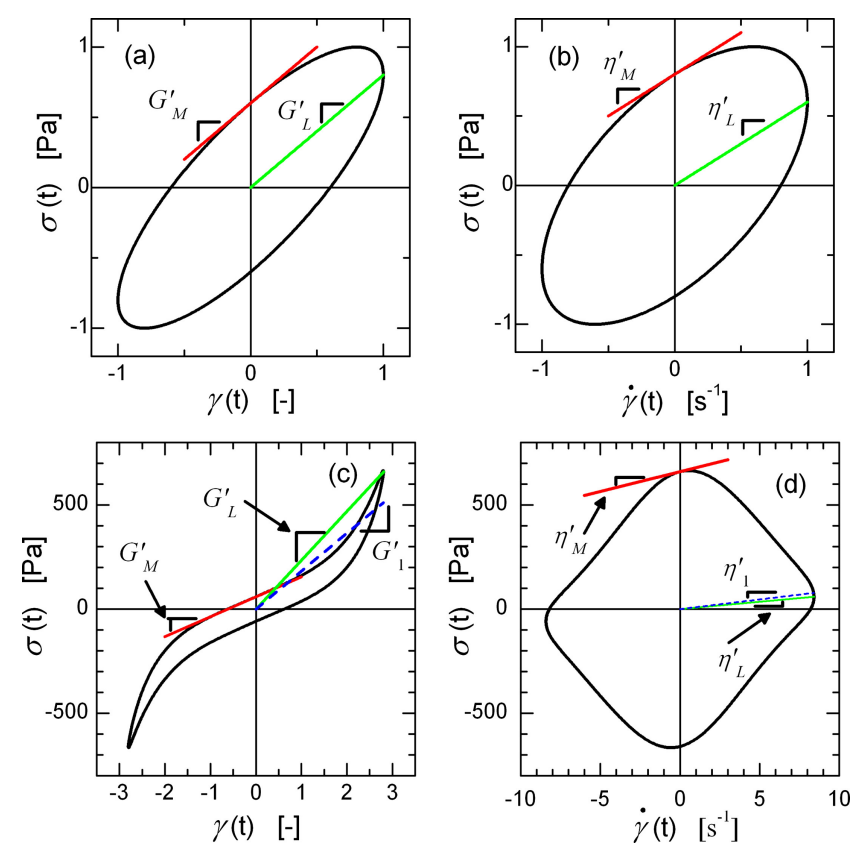

Figure 2.4: The visual definition of the viscoelastic moduli in Lissajous plots, extracted from (12)

$$
\left.G_{M}^{\prime} \equiv \frac{d \sigma}{d \gamma}\right|_{\gamma=0}=\sum_{n: o d d} n G_{n}^{\prime}
$$

and the large-strain modulus $\left(G_{L}^{\prime}\right)$ is the secant at maximum strain, hence, at zero shear-rate $(\dot{\gamma}(t)=0)$

$$
\left.G_{L}^{\prime} \equiv \frac{\sigma}{\gamma}\right|_{\gamma=\gamma_{a}}=\sum_{n: o d d} n G_{n}^{\prime}(-1)^{\frac{n-1}{2}}
$$

Meanwhile, the minimum-rate dynamic viscosity $\left(\eta_{M}^{\prime}\right)$ is the instantaneous viscosity at zero shear-rate

$$
\left.\eta_{M}^{\prime} \equiv \frac{d \sigma}{d \dot{\gamma}}\right|_{\dot{\gamma}=0}=\frac{1}{\omega} \sum_{n: o d d} n G_{n}^{\prime \prime}(-1)^{\frac{n-1}{2}}
$$

and the large-rate dynamic viscosity is the instantaneous viscosity at the largest $\dot{\gamma}$

$$
\left.\eta_{L}^{\prime} \equiv \frac{\sigma}{\dot{\gamma}}\right|_{\dot{\gamma}=\dot{\gamma}_{a}}=\frac{1}{\omega} \sum_{n: o d d} n G_{n}^{\prime \prime}
$$

From the definitions above, a parameter called Stiffening ratio $(S)$ may also be defined

$$
S=\frac{G_{L}-G_{M}}{G_{L}}
$$

The stiffening ratio indicates the behavior of the material: (i) $S=0$ indicates a linear elastic response; (ii) $S>0$ indicates intra-cycle strain stiffening, and (iii) $S<0$ indicates intra-cycle strain softening. Likewise, the shear tickening ratio $(T)$ may be defined as 


$$
T=\frac{\eta_{L}^{\prime}-\eta_{M}^{\prime}}{\eta_{L}^{\prime}}
$$

The shear thickening ratio indicates the behavior of the material: (i) $T=0$ indicates a linear viscous response; (ii) $S>0$ indicates intra-cycle shear thickening, and (iii) $S<0$ indicates intra-cycle shear thinning.

The behavior of pseudoplastic and elasto-viscoplastic materials, among others, was studied using the Chebyshev polynomials framework (14).

Dimitriou et al. (9) proposed a strain decomposition analysis, analogous to the stress decomposition analysis. It consists of splitting the strain output $\gamma$ into two additive parts, $\gamma=\gamma_{e}+\gamma_{v}$. From this analysis, the material functions $J_{M}^{\prime}, J_{L}^{\prime}, R, \phi_{M}^{\prime}, \phi_{L}^{\prime}$ and $F$ arise and are analogous to $G_{M}^{\prime}, G_{L}^{\prime}, S, \eta_{M}^{\prime}, \eta_{L}^{\prime}$ and $T$, respectively. For the sake of simplicity, the definition these properties will not be formally demonstrated in this document.

The previously mentioned approaches were reviewed and discussed by Rogers and Lettinga (45). The use of complex linear algebra to analyze LAOS responses was criticized. Based on the assumption that a more physically meaningful approach should be followed, Rogers et al. described the nonlinear oscillatory responses as a result of periodic sequences of physical processes (SPP) (44) and proposed the observation of the response wave in a 3D space formed by stress, strain and shear rate (42). The author claims that the distinction between elastic and viscous processes should not be based on the whole-waveform approach. Moreover, it may be seen as a more straightforward method since it does not rely on infinite series, opposed to the FT-Rheology and Stress Decomposition analysis techniques.

The SPP analysis relies on the definition of three vectors in the 3D space determined by stress, strain and shear rate: two reference vectors $s_{1}$ and $s_{2}$ and a vector $B$ normal to the curve. They are represented in figure 2.5 and mathematically given as follows

$$
\begin{array}{r}
s_{1}=[-1,0,0] \\
s_{2}=[0,0,1] \\
B^{\text {mod }}=\left[B_{\gamma}, \omega B_{\dot{\gamma}}, B_{\sigma}\right]
\end{array}
$$

Dynamic moduli $R^{\prime}$ and $R^{\prime \prime}$ are defined as the projections of the binomial vector $B$ into the strain and shear rate directions, respectively. 


$$
\begin{aligned}
& R^{\prime}\left(\gamma_{a}, \omega, t\right)=\left|G^{*}\left(\gamma_{a}, \omega, t\right)\right| \cos (\delta(t)) \\
& R^{\prime \prime}\left(\gamma_{a}, \omega, t\right)=\left|G^{*}\left(\gamma_{a}, \omega, t\right)\right| \sin (\delta(t))
\end{aligned}
$$

where $G^{*}$ is the complex modulus, $\delta$ is the phase angle and both may be obtained from the vectors described in equation 2-50. Notice that on the viscoelastic regime, the dynamic moduli $R^{\prime}$ and $R^{\prime \prime}$ reduce to $G^{\prime}$ and $G^{\prime \prime}$, respectively.

The SPP methodology succeeds to reduce the analysis of the nonlinear wave into two material functions without the need of a infinite number of coefficients that are difficult to attribute physical meaning. However, the coefficients $R^{\prime}$ and $R^{\prime \prime}$ are functions of the frequency, amplitude and time, while the previous methodologies were based in coefficients decoupled from time dependency.

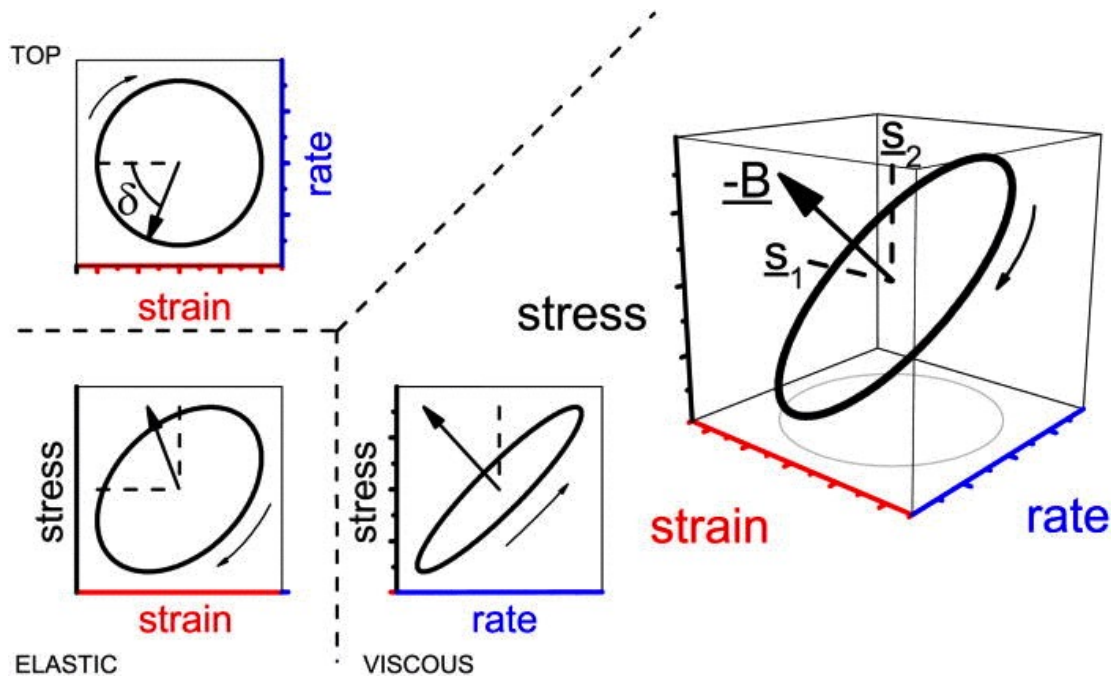

Figure 2.5: The visual representation of the SPP analysis, extracted from (42)

Each methodology has its perks and flaws. However, every LAOS analysis has a common problem which is the fact that the microscopic structure is constantly changing throughout the cycle. As it was previously stated, macroscopic and microscopic properties are intimately related to the microscopic structure. Therefore, they are also being instantaneously modified; and so is kinematic response, which is the key information to evaluate the previously mentioned properties (51). Since many variables are being simultaneously varied during the experiment, data becomes difficult to analyze. This problem comes from the fact that LAOS regime is a structure-changing motion, as it was previously mentioned in section 1.1; hence, this issue is unrelated to the approach used in the analysis. 


\subsubsection{1}

\section{LAOStrain}

LAOStrain are strain-controlled experiments out of the linear viscoelastic region. The deformation input wave has the following form

$$
\gamma(t)=\gamma_{a} \sin (\omega t)
$$

where $\gamma_{a}$ is a deformation amplitude large enough so that the stress response is no longer a linear function of the strain and shear rate at an oscillatory frequency $\omega$.

The main assumption of the SD decomposition proposed by Cho et al. (4) is that the total stress may be split into two additive components: $\sigma^{\prime}$, which is the elastic component, and $\sigma^{\prime \prime}$, which is the viscous component. These components were defined in equation 2-39. Comparing these terms to the definition of the Kelvin-Voigt parameters given by equations 2-14 and 2-15, it may be seen that

$$
G_{K V}=\frac{\sigma^{\prime}}{\gamma}=\Gamma^{\prime} \quad \text { and } \quad \eta_{K V}=\frac{\sigma^{\prime \prime}}{\dot{\gamma}}=\Gamma^{\prime \prime}
$$

Thus, the SD-generalized dynamic moduli are the Kelvin-Voigt elastic modulus and the Kelvin-Voigt viscosity. Another interesting result may be obtained from the viscoelastic moduli proposed by Ewoldt et al. (12) for the nonlinear regime, presented in equations 2-44, 2-45, 2-46 and 2-47.

Note that the author used Chebyshev polynomials as basis functions to create an orthogonal space, hence

$$
\left.G_{M}^{\prime} \equiv \frac{d \sigma}{d \gamma}\right|_{\gamma=0}=\left.\frac{d \sigma^{\prime}}{d \gamma}\right|_{\gamma=0}
$$

thus

$$
G_{M}^{\prime}=\left.G_{K V}\right|_{\gamma=0}+\left[\frac{d G_{K V}}{d \gamma} \gamma\right]_{\gamma=0}
$$

at last, since $\gamma=0$, we have

$$
G_{M}^{\prime}=G_{K V}(0)
$$

Analogously,

hence,

$$
\left.G_{L}^{\prime} \equiv \frac{\sigma}{\gamma}\right|_{\gamma=\gamma_{a}}=\left.\frac{\sigma^{\prime}}{\gamma}\right|_{\gamma=\gamma_{a}}+\left.\frac{\sigma^{\prime \prime}}{\gamma}\right|_{\gamma=\gamma_{a}}
$$

since $\dot{\gamma}=0$

$$
G_{L}^{\prime}=\left.G_{K V}\right|_{\gamma=\gamma_{a}}+\left.\eta_{K V} \frac{\dot{\gamma}}{\gamma}\right|_{\gamma=\gamma_{a}}
$$




$$
G_{L}^{\prime}=G_{K V}\left(\gamma_{a}\right)
$$

And the Kelvin-Voigt shear modulus evaluated at $\gamma=0$ and $\gamma=\gamma_{a}$ are, respectively, $G_{M}^{\prime}$ and $G_{L}^{\prime}$. Therefore, the quantity $S$ may be written as

$$
S \equiv \frac{G_{L}^{\prime}-G_{M}^{\prime}}{G_{L}^{\prime}}=\frac{G_{K V}\left(\gamma_{a}\right)-G_{K V}(0)}{G_{K V}\left(\gamma_{a}\right)}
$$

As it was previously stated, $S=0$ indicates a linear elastic response. This behavior may be clearly observed in equation 2-60 since $G_{K V}(0)=G_{K V}\left(\gamma_{a}\right)$ on the linear viscoelastic regime and this condition results in $S=0$.

Likewise, the same reasoning may be applied to $\eta_{M}^{\prime}$ and $\eta_{L}^{\prime}$

hence

$$
\left.\eta_{M}^{\prime} \equiv \frac{d \sigma}{d \dot{\gamma}}\right|_{\dot{\gamma}=0}=\left.\frac{d \sigma^{\prime \prime}}{d \dot{\gamma}}\right|_{\dot{\gamma}=0}
$$

$$
\eta_{M}^{\prime}=\left.\eta_{K V}\right|_{\dot{\gamma}=0}+\left.\frac{d \eta_{K V}}{d \dot{\gamma}} \dot{\gamma}\right|_{\dot{\gamma}=0}
$$

thus, since $\dot{\gamma}=0$

$$
\eta_{M}^{\prime}=\eta_{K V}(0)
$$

and

thus

$$
\left.\eta_{L}^{\prime} \equiv \frac{\sigma}{\dot{\gamma}}\right|_{\dot{\gamma}=\dot{\gamma}_{a}}=\left.\frac{\sigma^{\prime}}{\dot{\gamma}}\right|_{\dot{\gamma}=\dot{\gamma}_{a}}+\left.\frac{\sigma^{\prime \prime}}{\dot{\gamma}}\right|_{\dot{\gamma}=\dot{\gamma}_{a}}
$$

since $\gamma=0$

$$
\eta_{L}^{\prime}=\left.G_{K V} \frac{\gamma}{\dot{\gamma}}\right|_{\dot{\gamma}=\dot{\gamma}_{a}}+\left.\eta_{K V}\right|_{\dot{\gamma}=\dot{\gamma}_{a}}
$$

$$
\eta_{L}^{\prime}=\eta_{K V}\left(\dot{\gamma}_{a}\right)
$$

At last, the quatitiy $T$ may be written as

$$
T \equiv \frac{\eta_{L}^{\prime}=\eta_{M}^{\prime}}{\eta_{L}^{\prime}}=\frac{\eta_{K V}\left(\dot{\gamma}_{a}\right)-\eta_{K V}(0)}{\eta_{K V}\left(\dot{\gamma}_{a}\right)}
$$

Again, $T=0$ indicates a linear elastic response. This behavior may be clearly observed in equation 2-67 since $\eta_{K V}(0)=\eta_{K V}\left(\gamma_{a}\right)$ on the linear viscoelastic regime and this condition results in $T=0$.

As expected, it may be seen that the Kelvin-Voigt mechanical model is behind the analysis based on LAOStrain experiments.

\subsubsection{2}




\section{LAOStress}

LAOStress are stress-controlled experiments out of the linear viscoelastic region. The stress input wave has the following form

$$
\sigma(t)=\sigma_{a} \sin (\omega t)
$$

where $\sigma_{a}$ is a stress amplitude large enough so that the strain response is no longer a linear function of the stress at an oscillatory frequency $\omega$.

The strain decomposition method proposed by Dimitriou (9) is completely analogous to the SD decomposition method but decomposing the strain signal. Hence, we may state that the Maxwell mechanical model is behind the analysis based on LAOStress experiments.

$$
\begin{gathered}
J_{M}^{\prime}=J_{M W}(0) \\
J_{L}^{\prime}=J_{M W}\left(\sigma_{a}\right) \\
R \equiv \frac{J_{L}^{\prime}=J_{M}^{\prime}}{J_{L}^{\prime}}=\frac{J_{M W}\left(\sigma_{a}\right)-J_{M W}(0)}{J_{M W}\left(\sigma_{a}\right)} \\
\phi_{M}^{\prime}=\phi_{M W}(0) \\
\phi_{L}^{\prime}=\phi_{M W}\left(\sigma_{a}\right) \\
F \equiv \frac{\phi_{L}^{\prime}=\phi_{M}^{\prime}}{\phi_{L}^{\prime}}=\frac{\phi_{M W}\left(\sigma_{a}\right)-\phi_{M W}(0)}{\phi_{M W}\left(\sigma_{a}\right)}
\end{gathered}
$$

These relations will not be formally demonstrated in this document since they are completely analogous to the ones demonstrated above.

\subsection{2}

\section{Quasilinear viscoelasticity}

More recently, de Souza Mendes and Thompson (5) predicted the existence of a quasilinear LAOS regime, which was thereafter experimentally confirmed by Alicke (1) and de Souza Mendes et al. (6) for a commercial hair gel. The QL-LAOS regime occurs at high frequencies, namely when the cycle period is much shorter than the characteristic time of the material breakdown and buildup mechanisms. Under these conditions, there is no time for the material to rearrange its microscopic structure within a cycle. Thus, its structural level remains constant throughout the entire cycle. As it was previously stated in section 1.1, constant-structure motions, which are characterized by a sinusoidal response, occur either when the stress amplitude is not large enough to cause structural changes or the stress amplitude is large enough to cause structural changes but cycle period is shorter than the time scale of structural 
changes. While SAOS represents the former, QL-LAOS represents the latter. It is vital to understand that, unlike in SAOS linear regime, the material microscopic structure is not in its quiescent condition. Furthermore, each stress level results in a specific structural configuration. Therefore, even though SAOS and QL-LAOS analysis are intrinsically equivalent, material properties become a function of the stress level under QL-LAOS regime.

In section 2.2 , it was stated that linear viscoelasticity is defined as "the regime in small amplitudes in which the stress varies linearly with strain and shear rate. Also, material properties are independent of stress, strain and shear rate amplitudes throughout the entire regime." Neither of those conditions hold for the nonlinear viscoelastic region. However, the first condition is true here, while the second is not. Thus, this is called quasilinear region.

The analysis made in sections 2.2.1.1 for SAOStrain and 2.2.1.2 for SAOStress can be reproduced to QL-LAOStrain and QL-LAOStress, but the SAOS material functions $G^{\prime}(\omega), G^{\prime \prime}(\omega), J^{\prime}(\omega), J^{\prime \prime}(\omega), \phi^{\prime}(\omega)$ and $\phi^{\prime \prime}(\omega)$ would be replaced by $G^{\prime}\left(\omega, \gamma_{a}\right), G^{\prime \prime}\left(\omega, \gamma_{a}\right), J^{\prime}\left(\omega, \sigma_{a}\right), J^{\prime \prime}\left(\omega, \sigma_{a}\right), \phi^{\prime}\left(\omega, \sigma_{a}\right)$ and $\phi^{\prime \prime}\left(\omega, \sigma_{a}\right)$.

\subsubsection{1}

\section{Framework used to analyse materials under QL-LAOS}

The core of the experimental methodology of these previous works $(6,51,1)$ consists in obtaining material functions for a range of stress amplitudes $\sigma_{a}$ and frequencies $\omega$ through oscillatory tests, which may be time sweeps, stress sweeps, strain sweeps or frequency sweeps.

The time sweep is the most basic oscillatory experiment, since both $\sigma_{a}$ and $\omega$ are fixed. In this experiment, the material is subjected to a number of oscillation cycles and transient data is obtained. Hence, stress, strain and shear rate may be obtained as a function of time and the input and output waves may be reconstructed, as well as Lissajous plots. Meanwhile, stress sweeps are a sequence of time sweeps at different stress amplitudes, but at a fixed frequency. Even though transient data may also be obtained in stress sweeps, usually amplitude data is analyzed in this class of experiments. Strain sweeps are very similar to stress sweeps, but are performed in strain-controlled rheometers and strain amplitude is imposed to the material, instead of a stress amplitude. At last, frequency sweeps are analogous to the previous experiments, but consist of a sequence of time sweeps at different frequencies and at a fixed amplitude of stress or strain.

A strain-controlled rheometer was employed on these works, namely, the ARES-G2, manufactured by TA Instruments. According to the author, this choice stems from the fact that transducers are decoupled from the moving 
parts on this rheometer.

As frequency increases, the torque required to accelerate the geometry also increases, since the cycle period shortens. At high enough frequencies, the parcel responsible for the geometry's acceleration becomes relevant. On stress-controlled rheometers transducers are coupled with the moving parts and the stress value given by the rheometer, which is calculated based on the total torque, diverges from the real stress perceived by the sample. On straincontrolled rheometers, the inertial torque is not perceived by the transducers since they are decoupled from the moving parts.

A wide range of frequencies was investigated for the stress amplitudes of $\sigma_{a}=10,125$ and $312 \mathrm{~Pa}$. However, at each frequency, the strain amplitude that yields the aforementioned stress amplitudes is distinct. Therefore, strain sweeps were performed in order to obtain the relationship between stress and strain amplitudes for each frequency. Linear interpolation was employed to obtain the exact strain amplitude necessary to reach the required stress amplitude. Notice that, since a parallel plates geometry was employed, stress correction for inhomogeneous flow was necessary (38).

Thereafter, this strain amplitude value was input into a time sweep experiment at the corresponding frequency. Transient data obtained from these experiments yield information about the wave shapes, which was vital in order to prove the existence of the QL-LAOS regime. Lissajous plots were also created from this data and confirmed the results. The ratio between the third and first harmonics intensity $\left(I_{3} / I_{1}\right)$ was also used in order to prove the existence of the quasilinear regime as it complied to the linearity criteria $\left(I_{3} / I_{1}<3 \%\right)$ at high enough frequencies.

The use of transient time sweep experiments was necessary in these works due to the use of the waveshapes to prove the existence of the QL-LAOS regime. However, transient experiments requires a large amount of data and and requires a more complex and careful analysis. Furthermore, the use of time sweep experiments instead of stress, strain or frequency sweeps results in a huge number of rheometric experiments. Hence, a large volume of material is needed, as well as a lot of labour hours in the laboratory performing experiments and analyzing data.

\section{4}

\section{Beyond the classical approaches}

Classic material functions, as the ones described in sections 2.2.1.1 and 2.2.1.2, are usually perceived as entities unrelated to a particular class of mechanical behavior. However, we showed that every material function has 
constraints. Some of these properties originate from constitutive equations, thus are restricted to a class of materials that obey these equations (51).

It is clear that the viscosity $\eta$, which is defined as a ratio of the shear stress $\sigma$ to the shear rate $\dot{\gamma}$ in a pure shear flow, is based on the Newtonian model. The representation of the viscosity $\eta$ of a generic material as $\eta(\dot{\gamma}, t)$ highlights the deviation of the material behavior from the Newtonian behavior, where $\eta$ is represented by the constant value of $\mu$. Likewise, the shear modulus $G$ of a generic material is defined as the ratio of the shear stress $\sigma$ to the strain $\gamma$, which is inspired in the Hookean model and its representation as $G(\gamma, t)$ shows the deviation from the Hookean behavior, in which $G$ is also a constant value. Also, as it was demonstrated in sections 2.2.1.1 and 2.2.1.2, the oscillatory flow material functions arise from the Kelvin-Voigt model for storage and loss moduli $\left(G^{\prime}\right.$ and $\left.G^{\prime \prime}\right)$ and from the Maxwell model for compliances ( $J^{\prime}$ and $\left.J^{\prime \prime}\right)$ and fluidities $\left(\phi^{\prime}\right.$ and $\left.\phi^{\prime \prime}\right)$.

It is vital to expose the mechanical model employed in the definition of a material function since the constitutive model may be inadequate to describe the mechanical behavior of a certain class of materials. Since the model framework is usually implicit, many authors describe the mechanical behavior of a material employing material functions that are not suitable for that specific situation (51).

Thompson et al. (51) proposed a novel approach of rheological data analysis: the model based framework (MBFR). In the MBFR, the parameters of any given model become the material functions describing the desired mechanical behavior. Thus, the model employed in the definition of the material functions is always explicit and the constraints are more easily perceived.

The methodology consists on the determination of a constitutive model that qualitatively encompasses all possibilities of mechanical behavior for the material to be tested, followed by a conversion of model parameters into material functions. This conversion carries the test information in order to quantify the mechanical behavior.

As it was previously stated in section 2.1, the fundamentals of this methodology have already been used on this document when we exposed that the Maxwell liquid model was behind SAOStrain and LAOStrain analysis framework and the Kelvin-Voigt solid model was behind SAOStress and LAOStress analysis framework.

It is important to draw attention to the fact that neither the Maxwell nor the Kelvin-Voigt model are capable of qualitatively predicting the entire spectrum of mechanical behavior. An analysis whose framework is the Maxwell 
model is more suitable for fluids, while an analysis whose framework is the Kelvin-Voigt model is more suitable for solids.

The urge for a mechanical model that qualitatively encompasses the entire spectrum of mechanical behaviors is due to the fact that the mechanical behavior of a material to be tested is not known prior to its rheological characterization. If an unsuitable analysis framework is used to characterize the material, data that would reveal the mechanical behavior will be contaminated. Hence, the need to ensure that any given material will be qualitatively described by the mechanical model. Moreover, many materials of interest are soft solids, meaning that they behave as elastic or viscoelastic solids at low stresses and as viscoelastic or viscous fluids at higher stresses. A model that is able to describe both behaviors is vital in order to characterize these materials.

The simplest constitutive model that encompasses the entire spectrum of possible mechanical behaviors for a viscoelastic material is the Jeffreys model (51), whose analog is shown in figure 2.6.

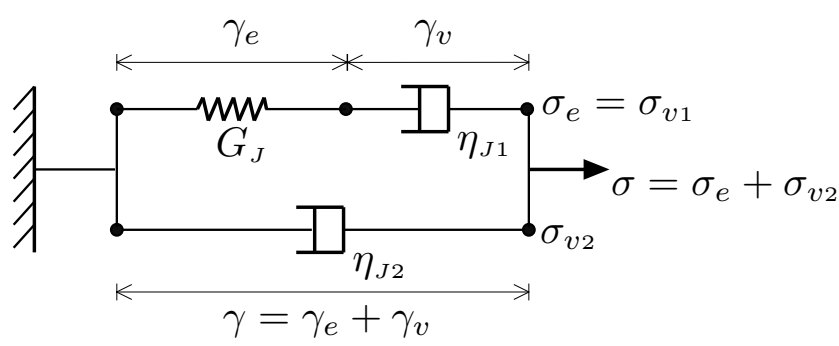

Figure 2.6: The Jeffreys mechanical analog.

Mathematically, it may be writen as

$$
\sigma+\frac{\eta_{1}}{G_{J}} \dot{\sigma}=\left(\eta_{J 1}+\eta_{J 2}\right) \dot{\gamma}+\eta_{J 1} \frac{\eta_{J 2}}{G_{J}} \ddot{\gamma}
$$

By analyzing either equation 2-75 or figure 2.6 , it can be seen that all previously cited models are particular cases of the Jeffreys model as it reduces to

- the Kelvin-Voigt model with $G_{K V}=G_{J}$ and $\eta_{K V}=\eta_{J 2}$, when $\eta_{J 1} \rightarrow \infty$;

- the Maxwell model with $G_{M W}=G_{J}$ and $\eta_{M W}=\eta_{J 1}$, when $\eta_{J 2}=0$;

- the Newtonian model with $\eta_{N W}=\eta_{J 1}+\eta_{J 2}$, when $G_{J} \rightarrow \infty$;

- the Hookean model with $G_{H K}=G_{J}$, when $\eta_{J 1} \rightarrow \infty$ and $\eta_{J 2}=0$.

Hence, this is a versatile model that may be used to describe all classes of materials embraced by other models presented on the previous sections. Another amazing feature that stems from this approach is that both stresscontrolled and strain-controlled experiments may be described by the same 
framework, since the Jeffreys mechanical analog encompasses both stress and strain wave decomposition.

The authors, however, restrict the use of this methodology to constantstructure motions. This restriction comes from the fact that, according to the authors, in structure-changing motions "stress varies periodically from zero to large values, and the microscopic state responds to this variation acoording to unknown properties and characteristics of the material. The desired information is the mechanical behavior, which varies with the configuration of the molecules or with the microstructural state, and thus varies periodically along the experiment in an unknown manner".

In order to obtain our model-based material functions, equation 2-75 may be rewritten as follows

$$
\sigma+\theta_{1} \dot{\sigma}=\eta\left(\dot{\gamma}+\theta_{2} \ddot{\gamma}\right)
$$

being $\theta_{1}$ the relaxation time, $\theta_{2}$ the retardation time and $\eta$ the steadystate shear viscosity. As it was stated on section 2.2, relaxation and retardation times derive from transient experiments. Further details on these properties are given on appendix A.

These material functions are related to the model parameters $\left(\eta_{J 1}, \eta_{J 2}\right.$ and $\left.G_{J}\right)$ as follows

$$
\eta=\eta_{J 1}+\eta_{J 2} ; \quad \theta_{1}=\left(1-\frac{\eta_{J 2}}{\eta}\right) \frac{\eta}{G} ; \quad \theta_{2}=\left(1-\frac{\eta_{J 2}}{\eta}\right) \frac{\eta_{J 2}}{G}
$$

It is shown in appendix $\mathrm{B}$ that $\theta_{1}, \theta_{2}, \eta_{J 1}, \eta_{J 2}$ and $G_{J}$ may be written as a function of J', J" and $\eta$, which are properties directly given by commercial rheometers:

$$
\begin{gathered}
\theta_{1}=\frac{\left(J^{\prime}+J^{\prime \prime}\right)}{J^{\prime}} \eta-\frac{J^{\prime \prime}}{J^{\prime} \omega} \\
\theta_{2}=\frac{\eta \omega J^{\prime \prime}-1}{\eta J^{\prime} \omega^{2}} \\
\eta_{J 1}=\frac{\eta^{2} \omega^{2} J^{\prime 2}+\left(\eta \omega J^{\prime \prime}-1\right)^{2}}{\eta \omega^{2}\left(J^{\prime 2}+J^{\prime \prime 2}\right)-\omega J^{\prime \prime}} \\
\eta_{J 2}=\frac{\eta \omega J^{\prime \prime}-1}{\eta \omega^{2}\left(J^{\prime 2}+J^{\prime \prime 2}\right)-\omega J^{\prime \prime}} \\
G_{J}=\frac{J^{\prime}\left(\eta^{2} \omega^{2} J^{\prime 2}+\left(\eta \omega J^{\prime \prime}-1\right)^{2}\right)}{\left(\eta \omega\left(J^{\prime 2}+J^{\prime \prime 2}\right)-J^{\prime \prime}\right)^{2}}
\end{gathered}
$$

Analogously, they may be written as a function of G', G" and $\eta$ :

$$
\begin{gathered}
\theta_{1}=\frac{\eta}{G^{\prime}}-\frac{G^{\prime \prime}}{G^{\prime} \omega} \\
\theta_{2}=\frac{G^{\prime \prime}}{G^{\prime} \omega}-\frac{G^{2}+G^{\prime 2}}{\eta G^{\prime} \omega^{2}}
\end{gathered}
$$




$$
\begin{gathered}
\eta_{J 1}=\eta-\frac{G^{\prime \prime}}{\omega}+\frac{G^{2}}{\eta \omega^{2}-G^{\prime \prime} \omega} \\
\eta_{J 2}=\frac{G^{\prime \prime}}{\omega}-\frac{G^{2}}{\eta \omega^{2}-G^{\prime \prime} \omega} \\
G_{J}=G^{\prime}\left[1+\frac{G^{2}}{\left(\eta \omega-G^{\prime \prime}\right)^{2}}\right]
\end{gathered}
$$

Notice that the material functions that arise from the model based framework are just the model parameters. In our case, the Jeffreys mechanical model parameters, which are allowed to vary with frequency and stress amplitude. The physical meaning of these material functions is the same of the parameters from which they originate, which are well known and qualitatively valid for the entire spectrum of possible mechanical behaviors for a viscoelastic material. 


\section{3}

\section{Experimental Set-Up and Methodology}

Two distinct fluids were used in this research: an elasto-viscoplastic commercial hair gel and a polymer dispersion. The former behaves as a linear viscoelastic solid at stresses below the yield stress $\sigma_{y}$, but irreversibly deforms and flows as a fluid for stresses above $\sigma_{y}$. Meanwhile, the latter is a $0.5 \%$ polyacrylamide dispersion in a $60 \%$ glycerin and $40 \%$ water base solution. At low stresses, the material is fully structured and viscosity tends to a constant value $\eta_{0}$. As polymer chains start to deform, break and move relative to one another, viscosity decreases until it reaches a constant value $\eta_{\infty}$, when the material is fully unstructured.

Since the microscopic structural state is determined by the stress perceived by the material, a stress-controlled rheometer was employed in this research. Namely, the AR-G2 rheometer, manufactured by TA Instruments was used. The AR-G2 motor and transducers are coupled in the upper fixture. Stress is calculated using the torque transmitted by the motor to the shaft, which is connected to the rotating geometry. Meanwhile, strain and shear rate are evaluated through the angular displacement, which is measured by the displacement transducer.

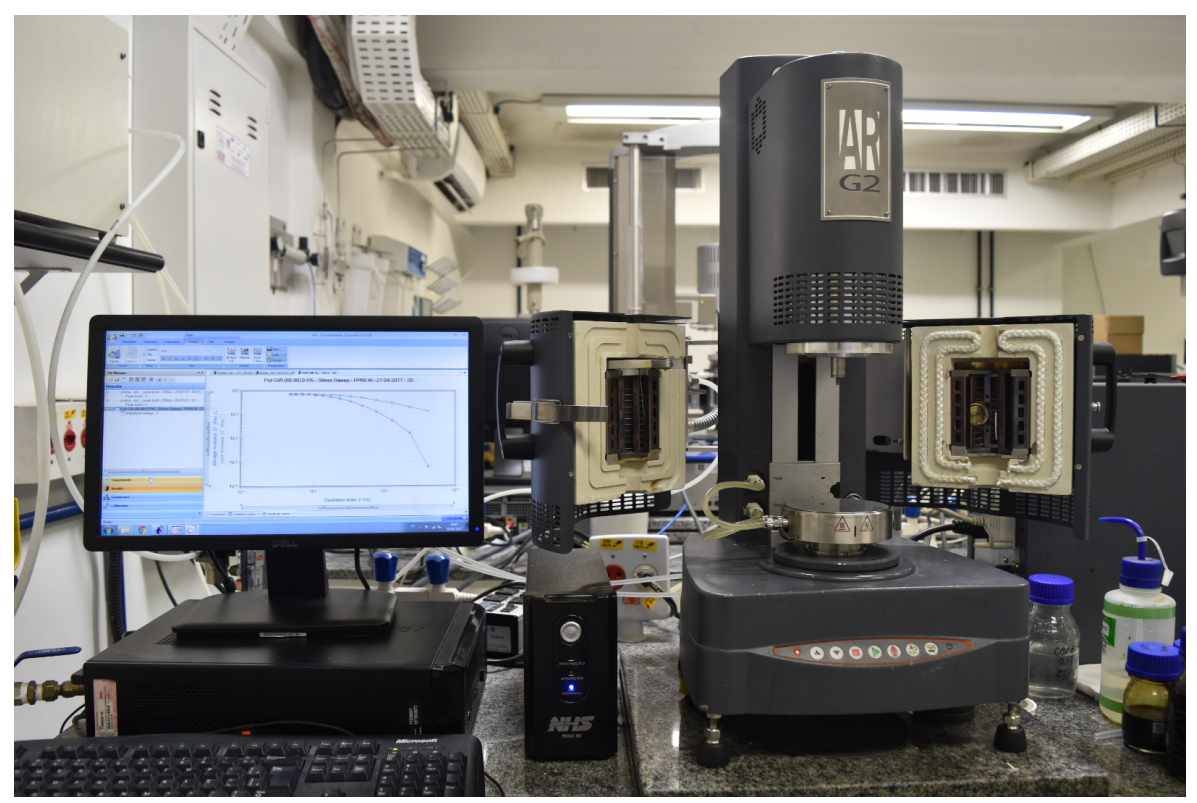

Figure 3.1: The stress-controlled AR-G2 rheometer, manufactured by TA Instruments. 
As it was previously mentioned in section 2.3.2.1, the torque is not entirely transmitted to the sample, since a parcel is consumed to overcome the instrument's inertia. This parcel becomes significant in oscillatory motion at high frequencies, since cycle periods are small and the instrument undergo huge accelerations. Hence, an inertial correction must be employed in this situation so that the stress applied to the sample is known and used to calculate the material functions (38).

For the gel, a $60 \mathrm{~mm}$ cross hatched parallel-plate geometry was employed since this material presents apparent wall-slip. As for the polyacrylamide dispersion, a $60 \mathrm{~mm}$ aluminum smooth parallel-plate geometry was employed. The aluminum plate is well suited to balance two difficulties about QL-LAOS experiments with this dispersion: low-torque values caused by low viscosity and high geometry-inertia at high frequencies.

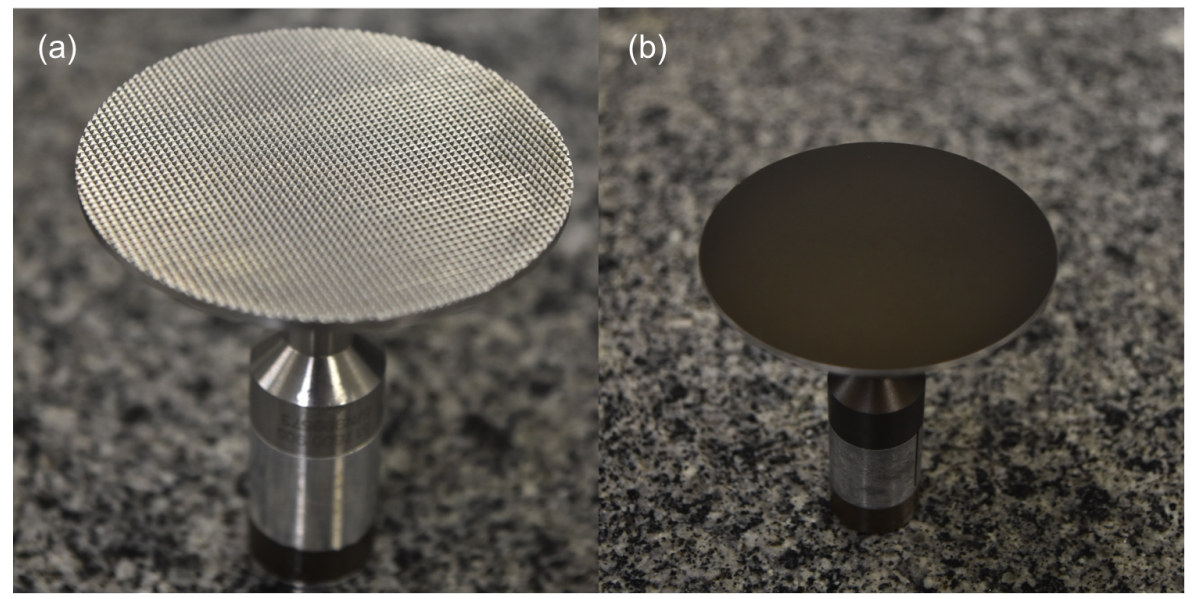

Figure 3.2: Details of the cross-hatched plate (a) and the aluminum plate (b).

Data obtained from experiments performed using parallel plate geometries should always be corrected due to inhomogeneous flow, as it will be further addressed in section 3.2. For steady-state experiments, WeissenbergRabinowicz correction (40) should be employed. Meanwhile, for oscillatory flow experiments, analogous correction developed by de Souza Mendes et al. (38) should be employed.

The model based framework described in section 2.4 will guide our analysis. From the equations presented on that section, it may be observed that very few experiments are necessary in order to obtain the base parameters to our material functions. Namely, a flow curve and a number of oscillatory experiments must be performed. While the first one provides the steady-state viscosity for different stress levels, the last provides the compliances, moduli and frequencies. Since the main goal of the experimental methodology is to reduce the number of rheometric experiments and the amount of data to be 
analyzed, a few stress sweeps will be performed instead of a huge number of time sweeps. Furthermore, no transient information will be analyzed.

\section{1}

\section{Rheometric experiments}

Sample placement is a vital step in every rheometric experiment, even though it is usually undervalued. If the sample placement is not carefully performed, data may not be representative of the material.

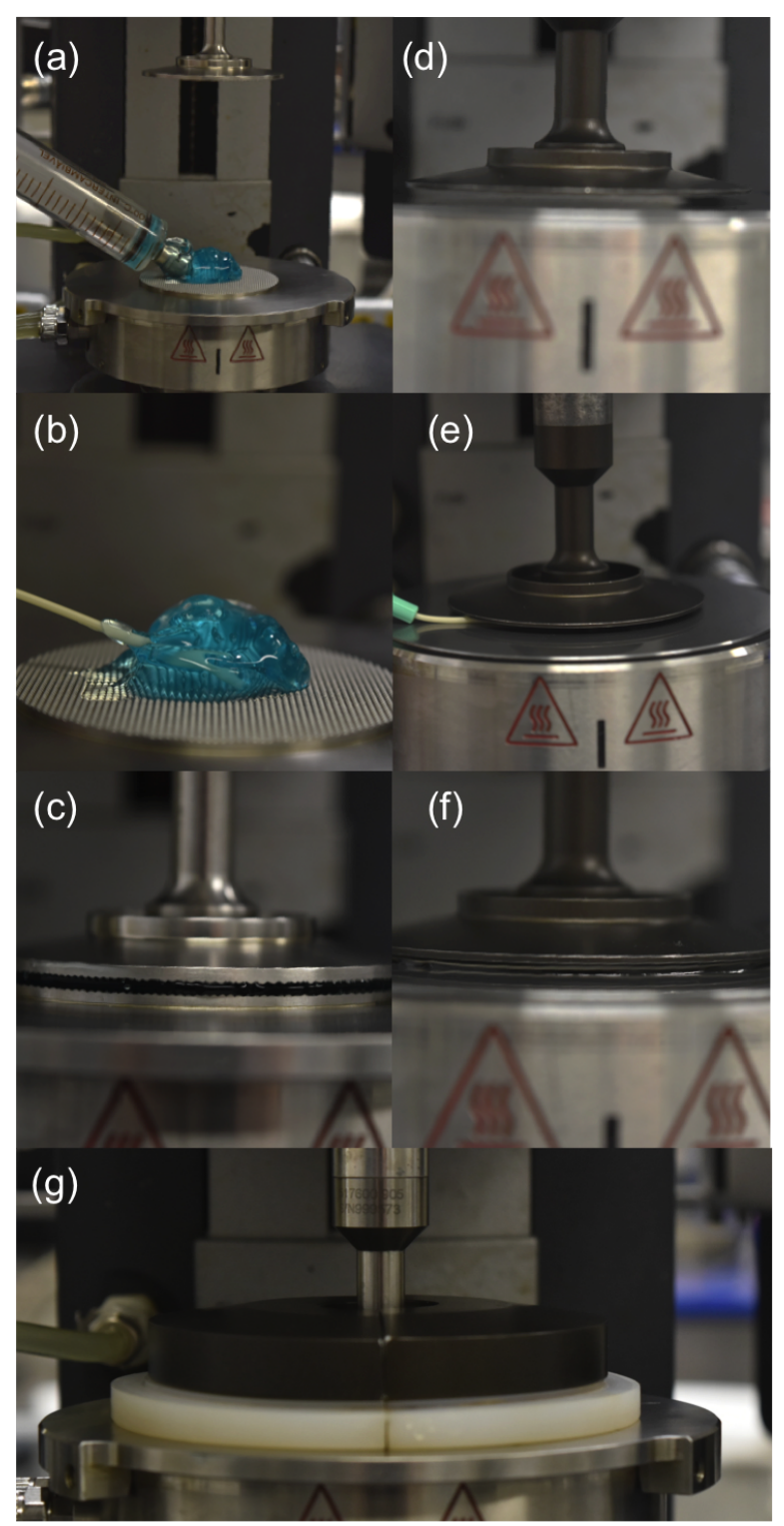

Figure 3.3: Details of the sample placement method for the gel ((a),(b),(c),(g)) and polyacrylamide solution $((\mathrm{d}),(\mathrm{e}),(\mathrm{f}),(\mathrm{g}))$.

For the commercial hair gel, the sample placement method was identical to the one performed by Alicke (1) in her work on QL-LAOS. As the author describes, "For every run, a new gel sample is used. The gel is loaded on 
the center of the lower plate with a glass syringe. Air bubbles need to be eliminated from the sample so as not to violate the continuum hypothesis, which states that the gap needs to be at least ten times bigger than the particle/droplet/bubble size. With the aid of an empty syringe, the bubbles are "sucked" from the sample, one bubble at a time. Once all bubbles are removed, the upper fixture is slowly lowered to avoid air entrapment and the gel spreads across the gap. When a $1.05 \mathrm{~mm}$ gap is reached, the sample is carefully trimmed with a cotton swab. Finally, the gap is set to $1.00 \mathrm{~mm}$. Then, a solvent trap cover is placed to minimize evaporation of the sample. Before testing, the sample is kept at rest for 10 minutes so that stabilization of the temperature and relaxation of the material." This method carefully avoids the most common problems caused by sample issues.

Similar sample placement method was employed in the polyacrylamide experiments. However, since there is no yield stress in this material, there are no bubbles to be "sucked" from the sample. Moreover, the absence of yield stress causes the material to flow when placed on the lower plate. Therefore, instead of placing the sample and lowering the upper fixture afterwards, the sample is directly placed between the plates at the trimming gap.

As it was previously stated, in order to obtain the necessary parameters for our analysis, only a flow curve and a number of stress sweeps at a range of frequencies are necessary. The flow curve consists on measuring the steadystate response of the material under a number of flow conditions, namely, a range of shear rates.

In order to ensure that the material reaches the steady-state before data is recorded, a peak hold experiment is previously performed: a constant shear rate is applied to the material and its response is measured as a function of time. The equilibration time required for the material to reach the steady-state is used as the input time to record data in the flow curve. The equilibration time is a function of the shear rate: as the shear rate decreases, the equilibration time increases. Hence, a peak hold experiment at the lowest shear rate to be tested in the flow curve is necessary to guarantee that every data point will be at the steady-state.

On the other hand, stress sweep experiments are not performed at steadystate since a sinusoidal oscillatory stress wave is imposed to the material. This test may be performed in "transient" or "correlation" modes. In the former, the response wave is measured with respect to time and non-linearities may be easily perceived by analyzing wave shapes. Meanwhile, the latter bear no information about the wave shapes. However, information about the phase angle and the input and output amplitudes are given in this mode. While 
transient data carry a lot of information about how the material behaves along the cycles, our goal is to determine the previously mentioned material functions. Transient data is not required in this case, thus our stress sweep experiments were performed in the correlation mode.

\section{2}

\section{Data processing}

Raw data must be processed before being input into the framework's equations. Data obtained from experiments performed using parallel plate geometries should always be corrected due to inhomogeneous flow. In contrast to the cone-and-plate or the narrow-gap concentric cylinder geometries, for which the deformation and shear-rate are uniform or nearly uniform throughout the sample, both these kinematic quantities vary linearly with the radial coordinate for the parallel-plate geometry.

$$
\gamma=\frac{\theta r}{h} \quad \text { and } \quad \dot{\gamma}=\frac{\dot{\theta} r}{h}
$$

where $h$ is the gap between the plates, $\theta$ is the angular displacement and $\dot{\theta}$ is the angular velocity.

When stress linearly varies with the radius, flow inhomogeneity is inconsequential. This is the case for Newtonian fluids, where $\sigma=\mu \dot{\gamma}$ and Hookean solids, where $\sigma=G \gamma$. Notice that stress in both cases may be written as $\sigma=C r$, where $C$ is a constant value.

The shear stress at the $\operatorname{rim}(r=R)$ for these materials may be obtained as follows

$$
M=\int_{0}^{R} r C r 2 \pi r d r=2 \pi C \int_{0}^{R} r^{3} d r=\frac{\pi R^{4} C}{2}
$$

since $\sigma_{R}=C R$ we obtain

$$
M=\frac{\pi R^{3}}{2} \sigma_{R}
$$

putting in terms of stress

$$
\sigma_{R}=\frac{2 M}{\pi R^{3}}
$$

Material properties may be evaluated at the rim conditions since strain and shear rate are also known at the rim.

For classes of materials in which stress does not vary linearly with the radius, flow analysis and rheometric theory are much more complex since flow inhomogeneity affects the material properties.

Flow inhomogeneity of the parallel-plate geometry may be accounted for by correcting the shear stress at the rim as given by the well-known Weissenberg-Rabinowitsch equation 


$$
\sigma=\frac{M}{2 \pi R^{3}}\left[3+\frac{\partial \ln M}{\partial \ln \dot{\gamma}_{R}}\right]
$$

For Newtonian fluids, $d \ln M / d \ln \dot{\gamma}_{R}=1$, and thus equation 3-5 reduces to equation 3-4. For non-Newtonian materials, the derivative needs to be evaluated through differentiation of experimental data. Notice that the WeissenbergRabinowitsch equation is only valid for steady-state flow.

Meanwhile, many stress correction equations were proposed for oscillatory flow. Most of them are dependent on the assumed mechanical model behind the framework $(48,39)$. However, an analogous correction to the one presented above was developed by de Souza Mendes et al. (38) and it is given as follows

$$
\sigma_{a}=\frac{M_{a}}{2 \pi R^{3}}\left[3+\frac{\partial \ln \left(M_{a} / 2 \pi R^{3}\right)}{\partial \ln \dot{\gamma}_{a, R}}\right]
$$

Therefore, equation 3-5 will be employed to correct steady-state flow data while equation 3-6 will be employed to correct oscillatory flow data.

Furthermore, oscillatory data needs to be revised at high frequencies and amplitudes due to high instrument inertia. For input stress waves at these conditions, the kinematic response will also be a high frequency and amplitude wave. In order to oscillate from zero to high strains in such a short timescale, the instrument undergo huge accelerations. Hence, the torque consumed to overcome the instrument's inertia becomes significant.

Raw data presented by the software includes a commanded stress value $\left(\sigma_{c m d}\right)$ estimated using the total torque as input; and another stress value $\left(\sigma_{a}\right)$ determined using the torque transmitted to the sample as input. In order to obtain the sample torque, the instrument deducts the inertial torque from the total torque, taking the phase shifts between the waves into consideration. The algebra behind the inertia correction is shown in appendix $\mathrm{C}$. We corrected $\sigma_{c m d}$ values using the equations presented in the appendix and compared to the $\sigma_{a}$ values presented by the software in order to validate the software corrected data. The agreement between them was notable, as presented in figure 3.4.

As it was previously shown, the model based material functions are a function of both stress amplitude $\sigma_{a}$ and frequency $\omega$. Therefore, our data points must be presented as iso-stress curves at a range of frequencies or isofrequency curves at a range of stresses so that the effect of each parameter is isolated and analyzed. When setting-up the experiment, we guarantee this by inputing the same conditions for each experiment. For example, a sweep from $10 \mathrm{~Pa}$ to $100 \mathrm{~Pa}$ with 3 points per decade using logarithmic distribution guarantees that data is recorded for stresses of $10.0 \mathrm{~Pa}, 21.5 \mathrm{~Pa}, 46.4 \mathrm{~Pa}, 100 \mathrm{~Pa}$ for every frequency. Therefore, data will be presented at the same stress levels 
for every frequency.

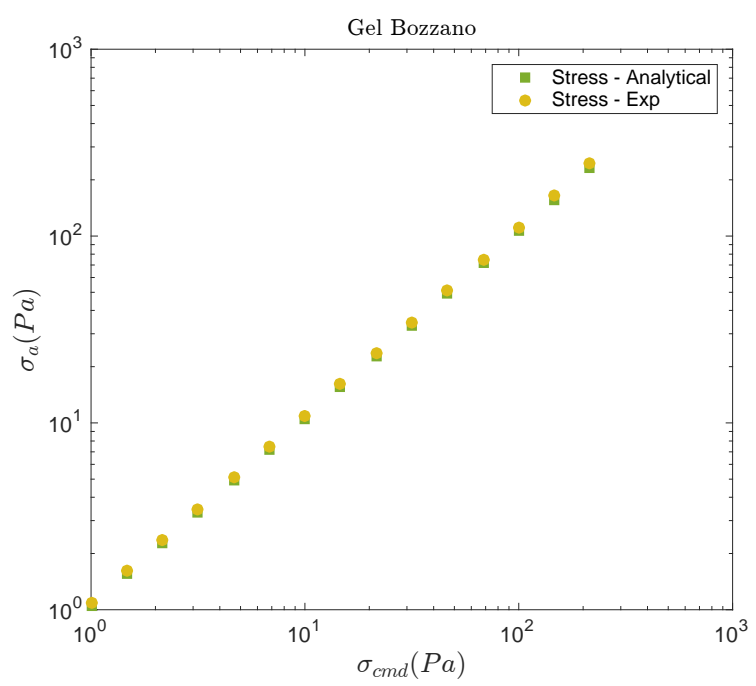

Figure 3.4: Comparison between the analytical and experimental corrected stress.

However, the stress corrections are amplitude and frequency dependent, generating distinct corrected stress values for each frequency. Hence, iso-stress curves may not be constructed from this data. In order to obtain an iso-stress curve for a range of frequencies, the desired stress amplitude need to be defined and, for each frequency, the properties at this amplitude will be calculated through linear interpolation between the two nearest data points. In order to guarantee that no significant error is introduced in this process, a large number of data points is required so that the distance between them is small enough for the linear approximation to be accurate.

At last, we need to verify if the flow conditions generate a constantstructure (SAOS/QL-LAOS) or structure-changing motion(LAOS). As it was previously stated, our analysis framework only comprises constant-structure motions, which are outlined by a linear or quasilinear response. Meanwhile, structure-changing motions are characterized by a nonsinusoidal response, which implies a nonlinear behavior. Since the stress is being imposed to the material and the kinematic response is being measured, nonlinearities may be quantified by measuring how nonlinear the kinematic response is. It is known that, besides the analysis of the wave shape, the magnitude of the higher odd harmonics of the output wave is a reliable measure of nonlinearity in oscillatory experiments $(22,18,23,57,58,60,4)$.

When setting-up stress sweep experiments in the "correlation mode" using the TRIOS software with the FT-Rheology package, it is possible to acquire harmonic intensities up to the $9^{\text {th }}$ order harmonic. When harmonic order increases, its contribution to the signal decreases. Thus, motion classification 
may be employed by comparing the first $\left(I_{1}\right)$ and third $\left(I_{3}\right)$ harmonic intensities. We've established the nonlinearity criteria as the threshold of $I_{3} / I_{1}>3 \%$. Hence, when this condition is attained for a pair of stress amplitude and frequency, this data point is at LAOS regime. Otherwise, it may be in SAOS or QL-LAOS regime.

The distinction between the two constant-structure motion regimes is made by checking if the data point is inside the linear viscoelastic region or not. The storage modulus $G^{\prime}$ is used to analyze if the material is in the linear viscoelastic region: an average of $G^{\prime}$ values for the three smaller amplitudes is compared to the value of $G^{\prime}$ for each data point. Since material properties in the linear viscoelastic region are not amplitude dependent, this averaged value may be considered as the linear viscoelastic value of the storage modulus. Notice that these three data points are necessarily in the linear viscoelastic region so that this method is valid. If the divergence between the averaged value and the storage modulus of a data point is more than $10 \%$, this data point is considered to be outside of the linear viscoelastic region.

After these data treatment processes, data may be applied to the equations for $\eta_{1}\left(\sigma_{a}, \omega\right), \eta_{2}\left(\sigma_{a}, \omega\right), G\left(\sigma_{a}, \omega\right), \theta_{1}\left(\sigma_{a}, \omega\right)$ and $\theta_{2}\left(\sigma_{a}, \omega\right)$ so that these material functions are obtained for each pair of stress amplitude and frequency. Thereafter, the iso-stress curves for each material function may be plotted. 


\section{4}

\section{Results and discussion}

In this section, the presented methodology will be applied in the characterization of the previously described materials. Through flow curves and stress sweep experiments, the Jeffreys-based QL-LAOS material functions will be obtained as a function of the frequency. Each curve will be obtained for a fixed stress amplitude.

\section{1}

\section{Flow Curve}

Flow curves are steady-state flow experiments in which the viscosity of the material is measured as a function of the shear rate. The stress applied to cause this shear rate to the material is also measured. For our analysis, the flow curve will provide a curve of the steady-state viscosity in relation to a stress level. All the results plotted below have already been corrected for inhomogeneous flow using the Weissenberg-Rabinowicz correction.

\subsection{1}

Gel

The steady-state shear flow results for the commercial hair gel are plotted in figure 4.1 .
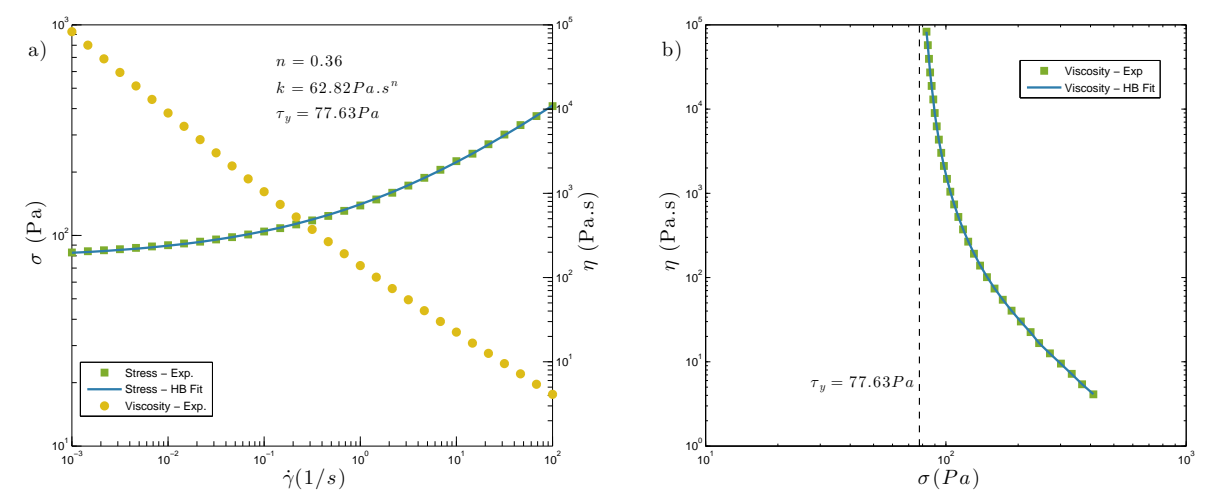

Figure 4.1: Flow curve obtained with a cross hatched parallel-plates geometry, for the commercial hair gel. 
In figure 4.1(a) the flow curve is presented in the most usual form, with stress and viscosity in the $\mathrm{y}$-axes and shear rate in the $\mathrm{x}$-axis. Meanwhile, the result is presented in a more convenient manner in figure 4.1(b) : the steadystate shear viscosity is given as a function of the shear stress. The Jeffreys-based material functions are determined for fixed shear stress levels and one of the parameters is the steady-state viscosity. Hence, it is advantageous to obtain the direct relation between these variables.

Both figures clearly exhibit the viscoplastic behavior of the gel, showing that there is a yield stress. Figure 4.1(a) shows that the Herschel-Bulkey equation $\left(\sigma=\sigma_{y}+k \dot{\gamma}^{n}\right)$ is a good fit for the hair gel. The parameters - namely, the yield stress $\sigma_{y}$, the consistency index $k$ and the power-law exponent $n$ - are indicated in this figure. Figure 4.1(b) clearly shows that the viscosity dramatically increases as the shear stress approaches the yield stress $\left(\sigma_{y}=77.63 \mathrm{~Pa}\right)$, indicating a solid-like behavior below this stress value.

\subsection{2}

\section{Polyacrylamide solution}

The steady-state shear flow results for the polyacrylamide dispersion are plotted in figure 4.2 .
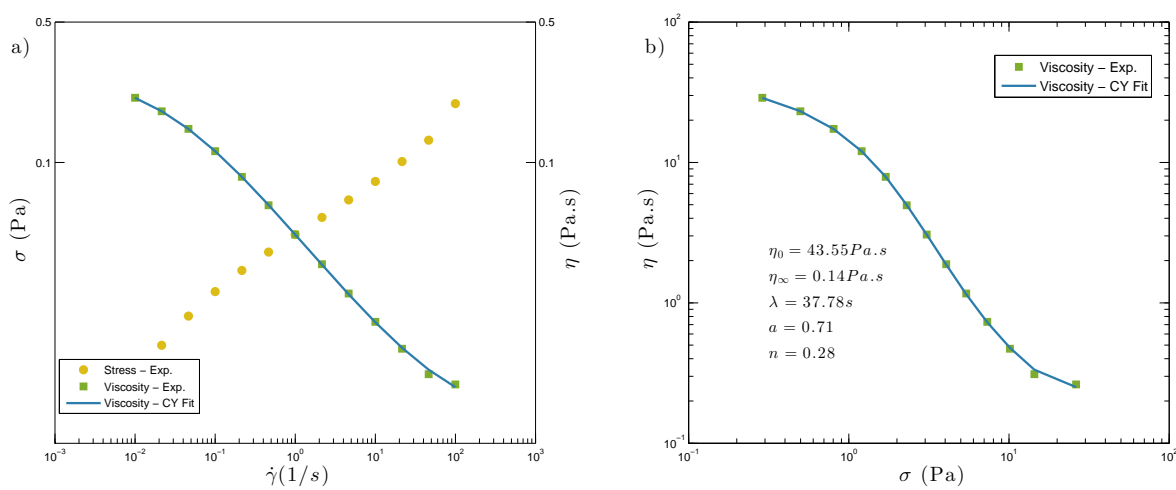

Figure 4.2: Flow curve obtained with a smooth parallel-plates geometry, for the polyacrylamide dispersion.

Again, the results are shown in two different manners. Figure 4.2(a) presents the classical approach, while figure 4.2(b) shows how the steadystate shear viscosity relates to the shear stress. Both plots show that CarreauYasuda is a good fit to represent the mechanical behavior of the polyacrylamide solution, presenting a tendency of reaching Newtonian plateaus at low shear stresses and shear rates $\left(\eta_{0}\right)$ and at high shear stresses and shear rates $\left(\eta_{\infty}\right)$. The former could not be attained since torque levels dropped below the 
rheometer's torque resolution before the plateau was reached, while the latter could not be reached due to sample spilling at high shear stresses.

The Carreau-Yasuda equation is given as follows:

$$
\eta=\eta_{\infty}+\left(\eta_{0}-\eta_{\infty}\right)\left(1+(\lambda \dot{\gamma})^{a}\right)^{\frac{n-1}{a}}
$$

where $\eta_{0}$ is viscosity at zero shear rate, $\eta_{\infty}$ is the viscosity at infinite shear rate, $\lambda$ is the relaxation time, $n$ is the power index and $a$ is a fit parameter.

\section{2}

\section{Stress Sweep}

For both materials, stress sweep experiments were conducted for a range of frequencies. It is worth noting that both inhomogeneous flow and inertia corrections were made in order to obtain results that correctly describe the material behavior under these conditions.

Data points may be mapped through a stress amplitude $\mathrm{x}$ frequency plot $\left(\sigma_{a} \mathrm{x} \omega\right)$. Each data point may be classified in SAOS/QL-LAOS or LAOS through it's $I_{3} / I_{1}$ ratio and in SAOS or LAOS/QL-LAOS verifying if the data point is within the linear viscoelastic region.

\subsection{1}

Gel

Stress sweeps were run for the following frequencies: $0.1,0.215,0.464$, 1.0, 2.15, 4.64 and 10Hz. Larger frequencies could not be reached due to inertia-dominated flow. When the raw phase angle is larger than $175^{\circ}$ the inertial effects are much higher than viscous and elastic effects. Therefore, small fluctuations in the instrument's inertia calibration may be as significant to the experiment results as the material's response. Hence, results are not reliable even after correction.

For each experiment, the commanded stress amplitude $\left(\sigma_{c m d}\right)$ ranged from $100 \mathrm{~Pa}$ to $1000 \mathrm{~Pa}$, with 12 points per decade. Higher stresses could not be reached due to sample spilling.

Figure 4.3 shows all the processing steps from raw data (figure 4.3(a)) to the fully processed data (figure 4.3(d)), presenting the distinction between the regimes (linear, nonlinear and quasilinear). As it was previously stated in section 3.2, data must be processed to account for inertial torque (figure 4.3(b)) and inhomogeneous flow between parallel plates (figure 4.3(c)).

Iso-stress curves could not be made from corrected data. Hence, linear interporlation was employed in order to obtain the material properties at 100, $120,140,160,180$ and 200Pa, which are the data points showed in figure 4.3(d). 

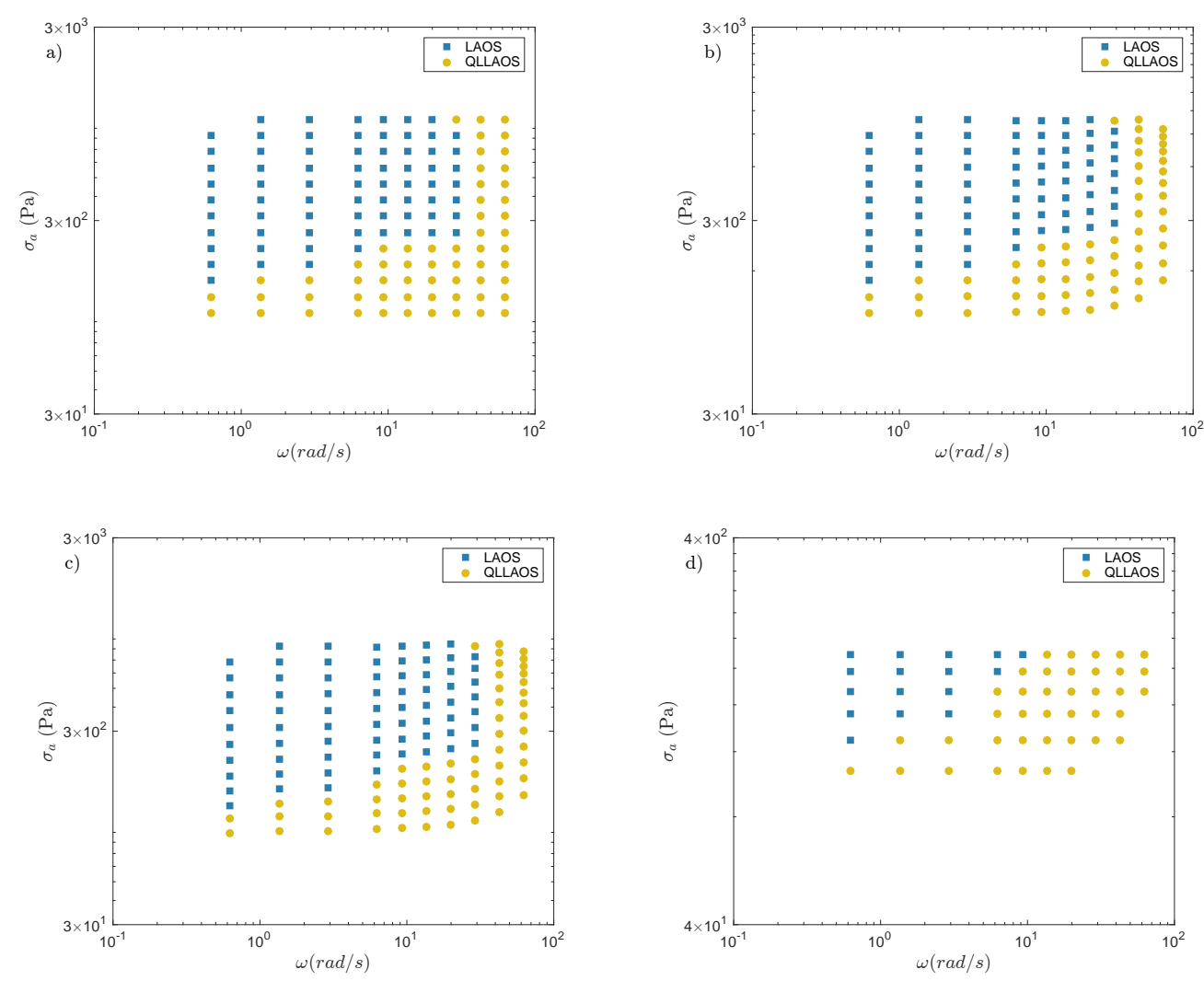

Figure 4.3: Linearity map for a) the raw data, b) data after inertia correction, c) data after inhomogeneous flow correction and d) fully processed data

The Jeffreys-based material functions were plotted for the data points showed in figure $4.3(\mathrm{~d})$.

It may be seen from figure 4.4(a) that $\eta_{1}$ is highly dependent of the stress amplitude, increasing as $\sigma_{a}$ decreases. This trend is expected since $\eta \rightarrow \infty$ as $\sigma \rightarrow \sigma_{y}$. Meanwhile, small fluctuations of $\eta_{1}$ may be observed in the frequency spectrum for a fixed stress amplitude.

For a perfect Jeffrey material, $\eta_{2}$ represents the viscosity of the completely unstructured material (i.e. $\eta_{\infty}$ ). However, figure $4.4(\mathrm{~b})$ depicts a distinct behavior since $\eta_{2}$ is dependent of the stress amplitude and frequency. This trend clearly shows that the hair gel behavior diverge from the model framework. Since the sum of the viscosities $\left(\eta_{1}\right.$ and $\left.\eta_{2}\right)$ is always equal to the steady-state shear viscosity $\eta$, which is a constant value, the fluctuations observed for $\eta_{1}$ are a compensation for the variations of $\eta_{2}$ in the frequency spectrum.

The comparison between the viscosity values gives a clear notion that $\eta_{1}$ is always much larger than $\eta_{2}$. This is expected since $\eta_{2}$ tends to represent $\eta_{\infty}$, which is the lowest viscosity value for shear-thinning materials. This huge gap between the viscosity values also clarifies the fact that the $\eta_{1}$ fluctuations in the frequency spectrum are almost negligible. 
The shear modulus is also shown to increase as frequency increases. This trend shows that the stiffness is much higher when the material is subjected to stimuli whose characteristic times are shorter. On the other hand, the material respond more rigidly to smaller stress amplitudes, meaning that the material is stiffer closer to the yield stress.
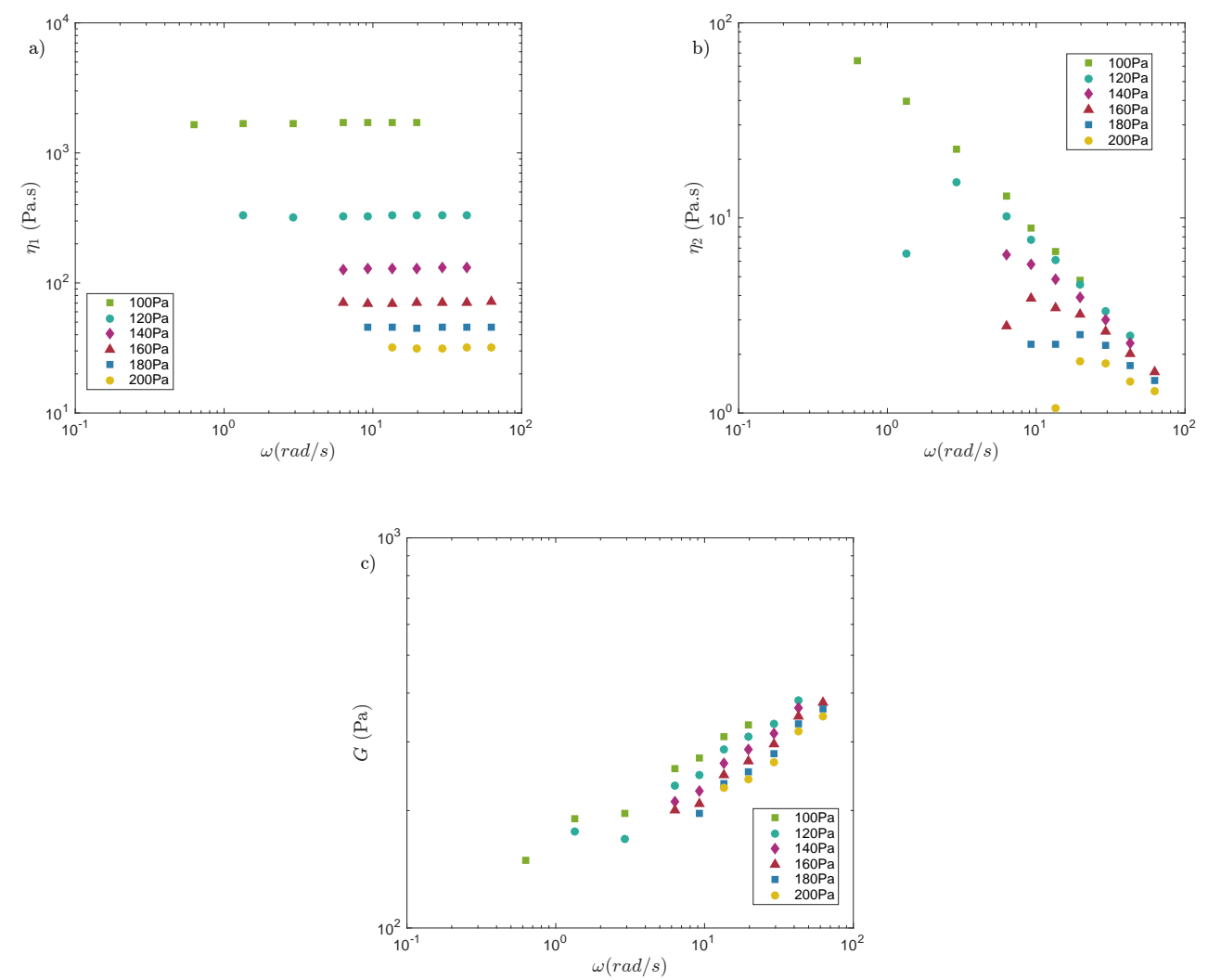

Figure 4.4: Jeffrey-based material functions for the commercial hair gel

The characteristic times $\theta_{1}$ and $\theta_{2}$ are represented in figures 4.5(a) and 4.5(b), respectively. Both properties decay as the stress amplitude increases, which shows that the elasticity decreases as the material becomes less structured. Furthermore, the relaxation time should tend to infinite below the yield stress, which is a trend shown by the results as $\theta_{1}$ exponentially increases as $\sigma_{a} \rightarrow \sigma_{y}$.

The presentation of these results is the highlight of this novel methodology since it clearly shows information about the material's elasticity and structural state at large amplitudes without the need of a complex mathematical analysis that overlook the physical phenomena that govern the flow.

As frequency is increased, both $\theta_{1}$ and $\theta_{2}$ decreases. It is worth noting that $\theta_{2}$ reaches negligible values. From the analog point of view, this is supported by the fact that at high enough frequencies, the analog branch that contains 
$\eta_{2}$ doesn't respond rapidly enough to the oscillations and the material behaves closer to a Maxwell fluid.

For a Maxwell fluid, there is only one time constant, which is described as follows:

$$
\theta_{1}=\frac{\eta_{1}}{G}
$$

Since $\eta_{1}$ is almost invariable in the frequency spectrum and $G$ increases as $\omega$ does the same, it is expected that $\theta_{1}$ decreases in this situation. As the Maxwell framework exhibits only one time constant, it is also consistent that $\theta_{2}$ is negligible.
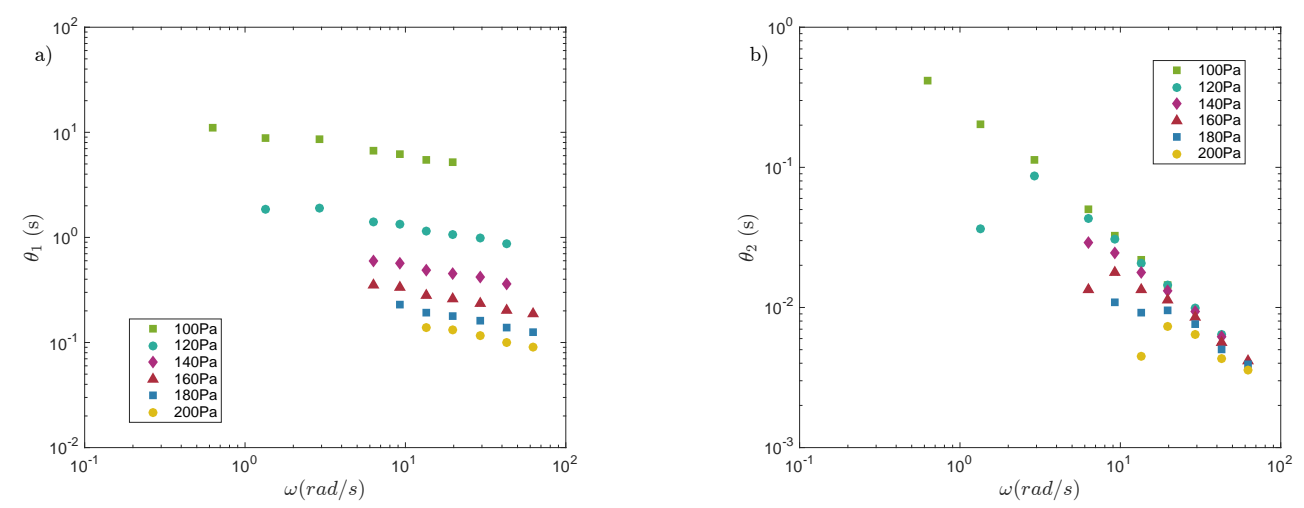

Figure 4.5: Time constants for the commercial hair gel

At smaller frequencies and larger stress amplitudes, the equations for $\eta_{2}$ and $\theta_{2}$ become very sensible to minor fluctuations on the values of $\eta, \omega, G^{\prime}$ and $G^{\prime \prime}$. Hence, this region presents some scattered data points on the retardation viscosity and retardation time plots.

\subsection{2}

\section{Polyacrylamide solution}

For the Polyacrylamide solution, stress sweeps were run for commanded stress amplitudes $\left(\sigma_{c m d}\right)$ ranging from 0.1 to $100 \mathrm{~Pa}$. Smaller stress values fall below the instrument's torque resolution, while higher stresses could not be reached due to sample spilling.

The following frequencies were input: $0.1,0.215,0.464,1.0,2.15$ and $4.64 \mathrm{~Hz}$. Again, larger frequencies evoke inertia-dominated flow and cannot be taken into account.

As it may be perceived from figure 4.6, the transition between small amplitude and large amplitude regimes is frequency dependent. This is due to the fact that in an oscillatory motion, the stress never persists at its maximum value $\sigma_{a}$. Thus, there is never enough time of continuous exposure to $\sigma_{a}$ that 
would be needed for the breakup process to be fully completed. As frequency increases, the continuous exposure time to higher stresses decreases. Hence, the breakup process becomes farther from completion. This fact, previously predicted by de Souza Mendes and Thompson (5), is usually ignored as researchers tend to designate a frequency-independent amplitude value for the transition between these regions.
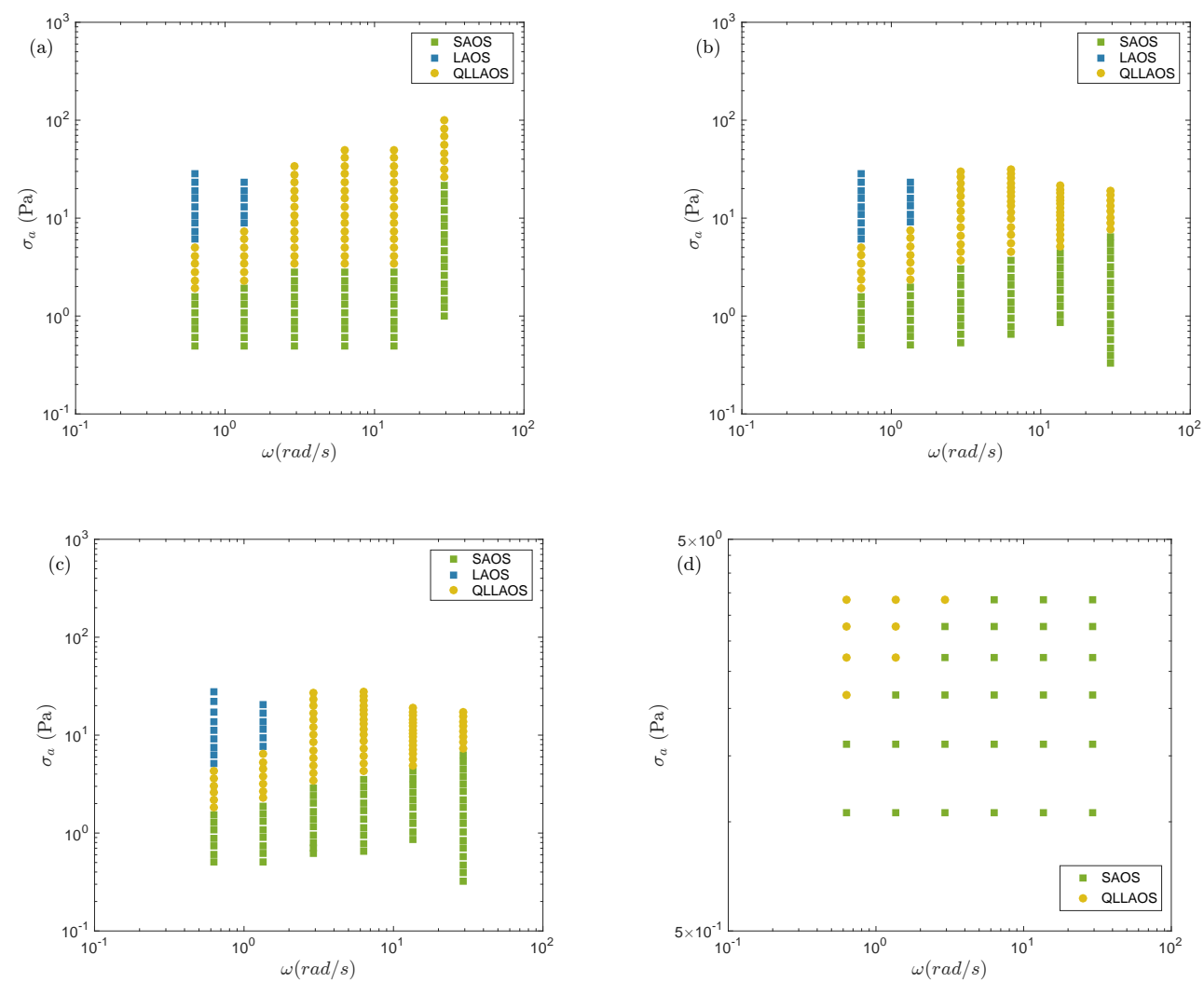

Figure 4.6: Linearity map for a) the raw data, b) data after inertia correction, c) data after inhomogeneous flow correction and d) fully processed data

Figure 4.7 demonstrates this frequency dependency in a different manner. Figure 4.7(a) shows that the storage modulus has distinct values at low frequencies for different stress amplitudes. As frequency increases, dispersion of the storage modulus values diminishes until they converge at large frequencies. As dispersion decreases, more stress amplitudes fall into SAOS regime. This behavior is present until every stress amplitude falls into the SAOS regime, as shown in figure $4.7(\mathrm{~b})$. 

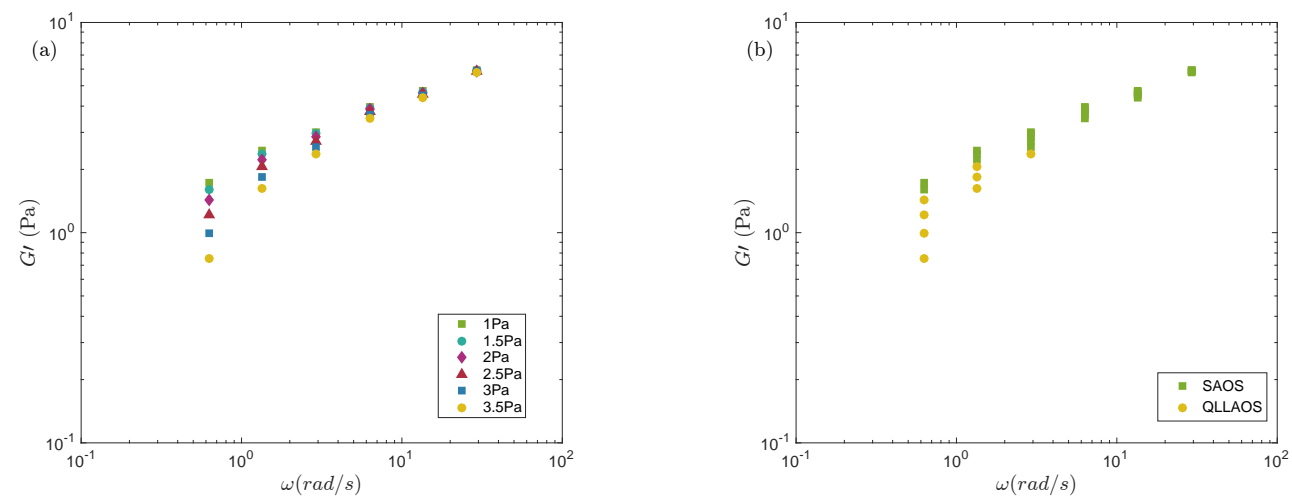

Figure 4.7: Dispersion of the storage modulus for distinct a) stress amplitudes and b) oscillatory regimes

The Jeffreys-based material functions were plotted for the following stress amplitudes: 1, 1.5, 2, 2.5, 3 and 3.5Pa.
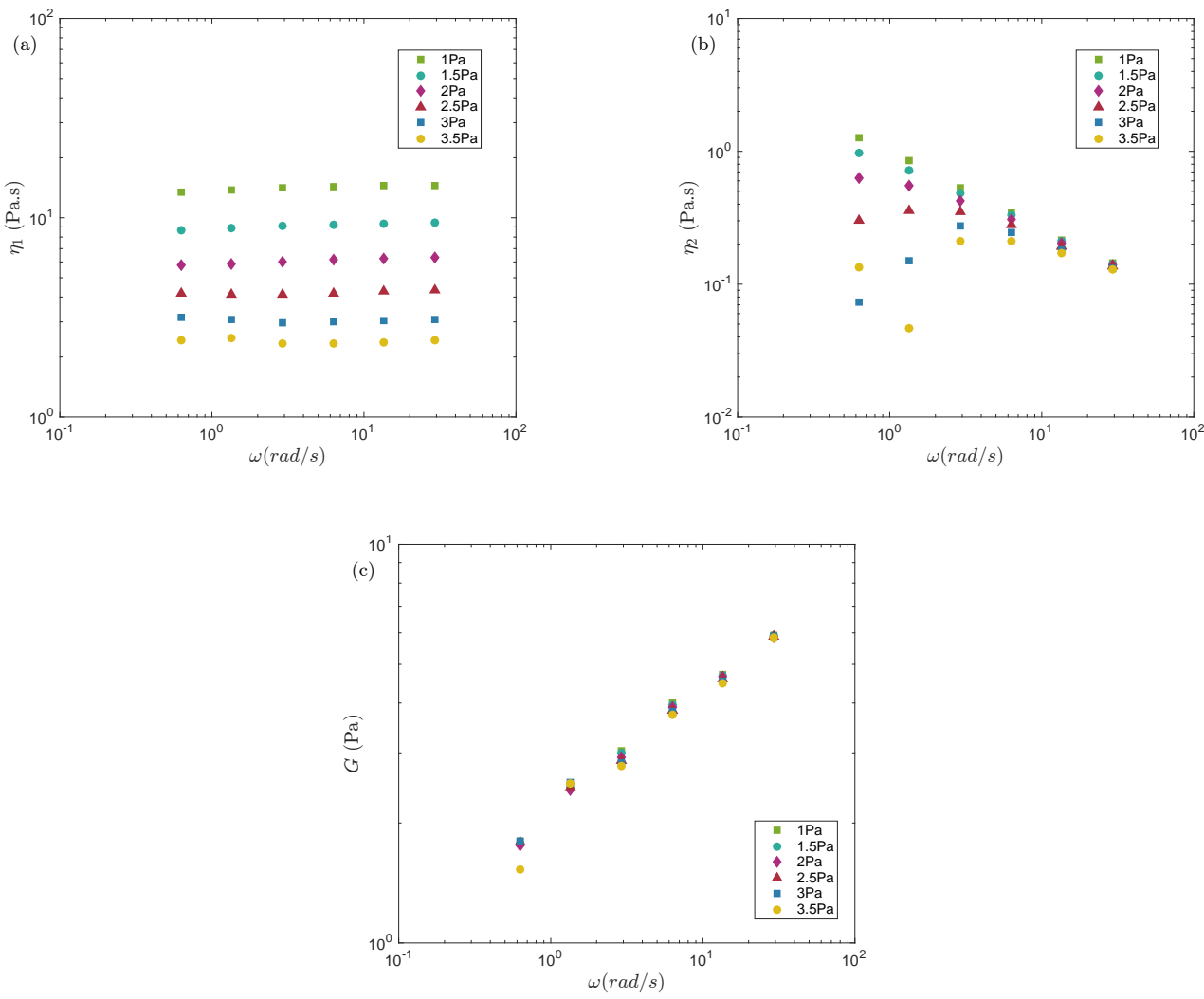

Figure 4.8: Jeffrey-based material functions for the polyacrylamide solution

Once more, $\eta_{1}$ rapidly decreases as the stress amplitude increases. This confirms the tendency of structure deconstruction shown in figure 4.2 as stress levels are raised. It is clear that $\eta_{1}$ is orders of magnitude higher than $\eta_{2}$, which tends to the $\eta_{\infty}$ value given by the Carreau-Yasuda fit when stresses and frequencies are high. Again, $G$ increases as frequency increases. Thus, the 
polyacrylamide solution responds more rigidly to stimuli whose characteristic times are shorter.
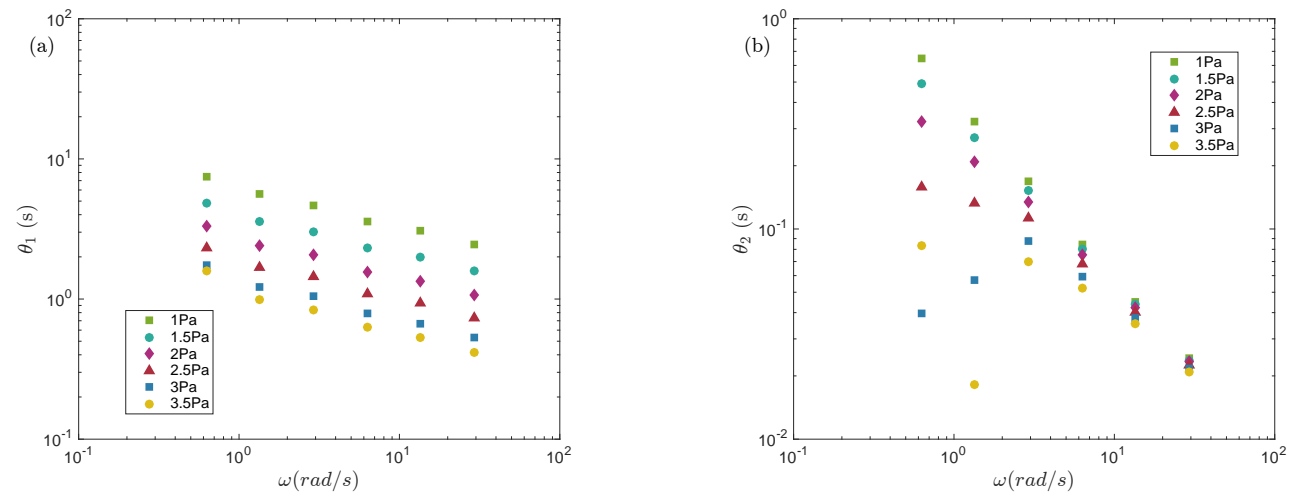

Figure 4.9: Time constants for the polyacrylamide solution

The same trends observed for the commercial hair gel time constants are presented on figure 4.9 for the polyacrylamide solution's $\theta_{1}$ and $\theta_{2}$. Both time constants decay with the increase of $\sigma_{a}$ and $\omega$, showing a decrease in elasticity when the material is less structured. Once more, the decrease of the time constants in the frequency spectrum allied with the fact that $\theta_{2}$ reaches negligible values suggests that the material verge upon a Maxwell fluid behavior at high frequencies.

Again, the sensibility of equations 2-84 and 2-86 for smaller frequencies and larger stress amplitudes causes scattered data points on the retardation viscosity and retardation time plots. 


\section{5}

\section{Final remarks}

In this work, we have developed a new methodology to analyze materials in constant-structure motion. Firstly, the selection of an appropriate framework is vital. Every framework has its model parameters and time constants. For the Jeffreys framework, there is a relaxation viscosity $\eta_{1}$, a retardation viscosity $\eta_{2}$, a shear modulus $G$, a relaxation time constant $\theta_{1}$ and a retardation time constant $\theta_{2}$. These parameters may be converted into material properties that are functions of the measurable quantities $\eta, G^{\prime}, G^{\prime \prime}, J^{\prime}$ and $J^{\prime \prime}$. It was shown that in order to obtain these quantities for a spectrum of various stress amplitudes and frequencies, only a steady-state flow sweep and a number of oscillatory stress sweeps at different fixed frequencies are necessary.

It is clear that the actual retardation and relaxation mechanisms within the microscopic structure of the tested materials differ from the ones predicted by the Jeffreys model. This is expected since any given framework is a simplistic modeling of a series of complex microscopic interactions. Nevertheless, the Jeffreys model is a much more suitable framework than the Maxwell or KelvinVoigt; which are usually implicit in most analysis since it encompasses the entire spectrum of mechanical behavior and represents the qualitative behavior of any given material.

The analysis of the Jeffreys-based material functions and time constants provides a reasonable perception of how the material behaves in the linear and quasilinear oscillatory flow regimes since qualitative information about the material microscopic structure may be obtained. It is also remarkable that both SAOS and QL-LAOS data may be analyzed and visualized without distinction.

Furthermore, data analysis presented on this work is straightforward as raw data obtained from the rheometer requires simple data processing steps before being input into the equations that evaluate the material functions. This fact contrasts with most large amplitude oscillatory shear analysis methods since they aim to analyze structure-changing motions and this requires complex mathematical manipulation of data.

At last, both experimental methodology and data analysis presented in this research are much simpler than the previous methodologies used to analyze materials in the quasilinear large amplitude oscillatory shear regime. 


\section{1}

\section{Future works}

The rheometric experiments have been reduced down to the simplest possible set of experiments. However, data processing is still necessary in order to obtain data for the same stress amplitudes at different frequencies. It is evident that, in overall, the methodology would become much simpler if no stress corrections were necessary.

This could be achieved if the rheometer's software employed the inertia and inhomogeneous flow corrections prior to data acquisition. However, this is not a simple task, since inertia correction uses the material phase angle, which is acquired during the experiment, and inhomogeneous flow correction uses the derivative of both torque and shear rate.

Inhomogeneous flow correction may be avoided if cone-plate or concentric-cylinder geometries are used. Even tough the latter is not recommended in oscillatory experiments due to a high inertia, the former may be suited for materials that present no apparent wall-slip. This possibility could be tested in future works, in order to render a simpler experimental methodology.

The analysis of constant-structure motion data may be further explored. The comparison between the Jeffrey's analysis and the Maxwell/Kelvin-Voigt analysis will be addressed in future works.

At last, it might also be interesting to employ mechanical models other than the Jeffreys. This would render different analysis that may be more suitable to some specific classes of materials. The suitability of the models could be evaluated through a parameter that captures the deviation of the real material in relation to the model's prediction. 


\section{Bibliography}

[1] ALICKE, A. A.. LAOS Rheological Characterization of an ElastoViscoplastic Material. Tese de Doutorado, PUC Rio, 2013.

[2] BARNES, H. A.. Thixotropy - a review. Journal of Non-Newtonian Fluid Mechanics, 70(1-2):1-33, may 1997.

[3] CAROtenuto, C.; GROSSO, M. ; MAFFETTONE, P. L.. Fourier transform rheology of dilute immiscible polymer blends: A novel procedure to probe blend morphology. Macromolecules, 41(12):4492-4500, jun 2008.

[4] CHO, K. S.; HYUN, K.; AHN, K. H. ; LEE, S. J.. A geometrical interpretation of large amplitude oscillatory shear response. Journal of Rheology, 49(3):747-758, may 2005.

[5] DE SOUZA MENDES, P. R.; THOMPSON, R. L.. A unified approach to model elasto-viscoplastic thixotropic yield-stress materials and apparent yield-stress fluids. Rheologica Acta, 52(7):673-694, apr 2013.

[6] DE SOUZA MENDES, P. R.; THOMPSON, R. L.; ALICKE, A. A. ; LEITE, R. T.. The quasilinear large-amplitude viscoelastic regime and its significance in the rheological characterization of soft matter. Journal of Rheology, 58(2):537-561, mar 2014.

[7] DEALY, J. M.; WISSBRUN, K.. Melt Rheology and Its Role in Plastics Processing: Theory and Applications. Springer, 1999.

[8] DIMITRIOU, C. J.. The rheological complexity of waxy crude oils: Yielding, thixotropy and shear heterogeneities. Tese de Doutorado, Massachusetts Institute of Technology, 2013.

[9] DIMITRIOU, C. J.; EWOLDT, R. H. ; MCKINLEY, G. H.. Describing and prescribing the constitutive response of yield stress fluids using large amplitude oscillatory shear stress (LAOStress). Journal of Rheology, 57(1):27-70, jan 2013. 
[10] DIMITRIOU, C. J.; MCKINLEY, G. H.. A comprehensive constitutive law for waxy crude oil: a thixotropic yield stress fluid. Soft Matter, 10(35):6619-6644, jun 2014.

[11] E., L.; VAN KEMPEN T.H.S.; F.P.T., B.; G.W.M., P. ; C.W.J., O.. Large amplitude oscillatory shear properties of human skin. Journal of the Mechanical Behavior of Biomedical Materials, 28:462-470, dec 2013.

[12] EWOLDT, R. H.; HOSOI, A. E. ; MCKINLEY, G. H.. New measures for characterizing nonlinear viscoelasticity in large amplitude oscillatory shear. Journal of Rheology, 52(6):1427-1458, nov 2008.

[13] EWOLDT, R. H.; HOSOI, A. E.; MCKINLEY, G. H.; CO, A.; LEAL, G. L.; COLBY, R. H. ; GIACOMIN, A. J.. An ontology for large amplitude oscillatory shear flow. In: AIP CONFERENCE PROCEEDINGS. AIP, 2008.

[14] EWOLDT, R. H.; WINTER, P.; MAXEY, J. ; MCKINLEY, G. H.. Large amplitude oscillatory shear of pseudoplastic and elastoviscoplastic materials. Rheologica Acta, 49(2):191-212, dec 2009.

[15] FletCHER, W. P.; GENT, A. N.. Nonlinearity in the dynamic properties of vulcanized rubber compounds. Rubber Chemistry and Technology, 27(1):209-222, mar 1954.

[16] GIACOMIN, A. J.; DEALY, J. M.. Using large-amplitude oscillatory shear. In: RHEOLOGICAL MEASUREMENT, p. 327-356. Springer Netherlands, 1998.

[17] GIACOMIN, A. J.; JEYASEELAN, R. S.; SAMURKAS, T. ; DEALY, J. M.. Validity of separable BKZ model for large amplitude oscillatory shear. Journal of Rheology, 37(5):811-826, sep 1993.

[18] GIACOMIN, A. J.; OAKLEY, J. G.. Structural network models for molten plastics evaluated in large amplitude oscillatory shear. Journal of Rheology, 36(8):1529-1546, nov 1992.

[19] GURNON, A. K.; WAGNER, N. J.. Large amplitude oscillatory shear (LAOS) measurements to obtain constitutive equation model parameters: Giesekus model of banding and nonbanding wormlike micelles. Journal of Rheology, 56(2):333-351, mar 2012.

[20] HYUN, K.; AHN, K. H.; LEE, S. J.; SUGIMOTO, M. ; KOYAMA, K.. Degree of branching of polypropylene measured from fourier-transform rheology. Rheologica Acta, 46(1):123-129, may 2006. 
[21] HYUN, K.; NAM, J. G.; WILHELLM, M.; AHN, K. H. ; LEE, S. J.. Large amplitude oscillatory shear behavior of PEO-PPO-PEO triblock copolymer solutions. Rheologica Acta, 45(3):239-249, aug 2005.

[22] HYUN, K.; WILHELM, M.; KLEIN, C. O.; CHO, K. S.; NAM, J. G.; AHN, K. H.; LEE, S. J.; EWOLDT, R. H. ; MCKINLEY, G. H.. A review of nonlinear oscillatory shear tests: Analysis and application of large amplitude oscillatory shear (LAOS). Progress in Polymer Science, 36(12):1697-1753, dec 2011.

[23] JEYASEELAN, R. S.; GIACOMIN, A.. Best fit for differential constitutive model parameters to non-linear oscillation data. Journal of Non-Newtonian Fluid Mechanics, 47:267-280, jun 1993.

[24] KLEIN, C. O.; SPIESS, H. W.; CALIN, A.; BALAN, C. ; WILHELM, M.. Separation of the nonlinear oscillatory response into a superposition of linear, strain hardening, strain softening, and wall slip response. Macromolecules, 40(12):4250-4259, jun 2007.

[25] KRIEGER, I. M.; NIU, T.-F.. A rheometer for oscillatory studies of nonlinear fluids. Rheologica Acta, 12(4):567-571, dec 1973.

[26] LÄUGER, J.; STETTIN, H.. Differences between stress and strain control in the non-linear behavior of complex fluids. Rheologica Acta, 49(9):909-930, apr 2010.

[27] LEBLANC, J. L.. Poly(vinyl chloride)-green coconut fiber composites and their nonlinear viscoelastic behavior as examined with fourier transform rheometry. Journal of Applied Polymer Science, 101(6):3638-3651, 2006.

[28] LEBLANC, J. L.; DE LA CHAPELLE, C.. Characterizing gum elastomers by fourier transform rheometry. Rubber Chemistry and Technology, 76(4):979-1000, sep 2003.

[29] MELITO, H.; DAUBERT, C. ; FOEGEDING, E.. Validation of a large amplitude oscillatory shear protocol. Journal of Food Engineering, 113(1):124-135, nov 2012.

[30] MELITO, H.; DAUBERT, C. ; FOEGEDING, E.. Relationships between nonlinear viscoelastic behavior and rheological, sensory and oral processing behavior of commercial cheese. Journal of Texture Studies, 44(4):253-288, mar 2013. 
[31] MEWIS, J.; WAGNER, N. J.. Thixotropy. Advances in Colloid and Interface Science, 147-148:214-227, mar 2009.

[32] MUJUMdAR, A.; BERIS, A. N. ; METZNER, A. B.. Transient phenomena in thixotropic systems. Journal of Non-Newtonian Fluid Mechanics, 102(2):157-178, feb 2002.

[33] NEIDHÖFER, T.; SIOULA, S.; HADJICHRISTIDIS, N. ; WILHELM, M.. Distinguishing linear from star-branched polystyrene solutions with fourier-transform rheology. Macromolecular Rapid Communications, 25(22):1921-1926, nov 2004.

[34] NEIDHÖFER, T.; SIOULA, S.; HADJICHRISTIDIS, N. ; WILHELM, M.. Distinguishing linear from star-branched polystyrene solutions with fourier-transform rheology. Macromolecular Rapid Communications, 25(22):1921-1926, nov 2004.

[35] NEIDHÖFER, T.; WILHELM, M. ; DEBBAUT, B.. Fourier-transform rheology experiments and finite-element simulations on linear polystyrene solutions. Journal of Rheology, 47(6):1351-1371, nov 2003.

[36] NEIDHÖFER, T.; WILHELM, M. ; DEBBAUT, B.. Fourier-transform rheology experiments and finite-element simulations on linear polystyrene solutions. Journal of Rheology, 47(6):1351-1371, nov 2003.

[37] OZKAN, S.; GILLECE, T. W.; SENAK, L. ; MOORE, D. J.. Characterization of yield stress and slip behaviour of skin/hair care gels using steady flow and LAOS measurements and their correlation with sensorial attributes. International Journal of Cosmetic Science, 34(2):193-201, jan 2012.

[38] P. R. DE SOUZA MendeS, A. A. A.; THOMPSON, R. L.. Parallel-plate geometry correction for transient rheometric experiments. 2014.

[39] PHAN-THIEN, N.; NEWBERRY, M. ; TANNER, R. I.. Non-linear oscillatory flow of a soft solid-like viscoelastic material. Journal of Non-Newtonian Fluid Mechanics, 92(1):67-80, aug 2000.

[40] RABINOWITSCH, B.. Über die viskosität und elastizität von solen. Zeitschrift für Physikalische Chemie, 145A(1):1-26, jan 1929.

[41] RAGHAVAN, S. R.; KHAN, S. A.. Shear-induced microstructural changes in flocculated suspensions of fumed silica. Journal of Rheology, 39(6):1311-1325, nov 1995. 
[42] ROGERS, S. A.. A sequence of physical processes determined and quantified in LAOS: An instantaneous local 2d/3d approach. Journal of Rheology, 56(5):1129-1151, sep 2012.

[43] ROGERS, S. A.. In search of physical meaning: defining transient parameters for nonlinear viscoelasticity. Rheologica Acta, 56(5):501525, apr 2017.

[44] ROGERS, S. A.; ERWIN, B. M.; VLASSOPOULOS, D. ; CLOITRE, M.. A sequence of physical processes determined and quantified in LAOS: Application to a yield stress fluid. Journal of Rheology, 55(2):435-458, mar 2011.

[45] ROGERS, S. A.; LETTINGA, M. P.. A sequence of physical processes determined and quantified in large-amplitude oscillatory shear (LAOS): Application to theoretical nonlinear models. Journal of Rheology, 56(1):1-25, jan 2012.

[46] SCHIMANSKY-GEIER, L.; MIETH, M.; ROSÉ, H. ; MALCHOW, H.. Structure formation by active brownian particles. Physics Letters $A$, 207(3-4):140-146, oct 1995.

[47] SIM, H. G.; AHN, K. H. ; LEE, S. J.. Large amplitude oscillatory shear behavior of complex fluids investigated by a network model: a guideline for classification. Journal of Non-Newtonian Fluid Mechanics, 112(2-3):237-250, jun 2003.

[48] SOSKEY, P. R.; WINTER, H. H.. Large step shear strain experiments with parallel-disk rotational rheometers. Journal of Rheology, 28(5):625-645, oct 1984.

[49] TAN, K.; CHENG, S.; JUGÉ, L. ; BILSTON, L. E.. Characterising soft tissues under large amplitude oscillatory shear and combined loading. Journal of Biomechanics, 46(6):1060-1066, apr 2013.

[50] TEN BRINKE, A. J. W.; BAILEY, L.; LEKKERKERKER, H. N. W. ; MAITLAND, G. C.. Rheology modification in mixed shape colloidal dispersions. part II: mixtures. Soft Matter, 4(2):337-348, 2008.

[51] THOMPSON, R. L.; ALICKE, A. A. ; DE SOUZA MENDES, P. R.. Modelbased material functions for SAOS and LAOS analyses. Journal of Non-Newtonian Fluid Mechanics, 215:19-30, jan 2015. 
[52] VAN DER VAART, K.; DEPYPERE, F.; GRAEF, V. D.; SCHALL, P.; FALL, A.; BONN, D. ; DEWETTINCK, K.. Dark chocolate's compositional effects revealed by oscillatory rheology. European Food Research and Technology, 236(6):931-942, mar 2013.

[53] VAN DUSSCHOTEN, D.; WILHELM, M.. Increased torque transducer sensitivity via oversampling. Rheologica Acta, 40(4):395-399, jul 2001.

[54] VITTORIAS, I.; WILHELM, M.. Application of FT rheology to industrial linear and branched polyethylene blends. Macromolecular Materials and Engineering, 292(8):935-948, aug 2007.

[55] WILHELM, M.. Fourier-transform rheology. Macromolecular Materials and Engineering, 287(2):83-105, feb 2002.

[56] WILHELM, M.. New methods for the rheological characterization of materials. Chemical Engineering and Processing: Process Intensification, 50(5-6):486-488, may 2011.

[57] WILHELM, M.; MARING, D. ; SPIESS, H.-W.. Fourier-transform rheology. Rheologica Acta, 37(4):399-405, aug 1998.

[58] WILHELM, M.; REINHEIMER, P. ; ORTSEIFER, M.. High sensitivity fourier-transform rheology. Rheologica Acta, 38(4):349-356, oct 1999.

[59] WILHELM, M.; REINHEIMER, P.; ORTSEIFER, M.; NEIDHÖFER, T. ; SPIESS, H.-W.. The crossover between linear and non-linear mechanical behaviour in polymer solutions as detected by fouriertransform rheology. Rheologica Acta, 39(3):241-246, aug 2000.

[60] YU, W.; WANG, P. ; ZHOU, C.. General stress decomposition in nonlinear oscillatory shear flow. Journal of Rheology, 53(1):215-238, jan 2009. 
A

\section{Transient experiments}

\section{A.1 \\ Creep}

To perform a creep experiment, a constant stress $\sigma$ must be applied to the material at the time $t=0$. For every material, the total deformation of the material will be a function of the time $(\gamma(t))$ as molecular and microscopic bonds are broken and relative movement between molecules and particles is initiated. After a long period of time, total deformation will reach a plateau if the material possesses a solid-like behavior. On the other hand, deformation will continuously increase at a constant rate if the material possesses a fluid-like behavior, as shown in figure A.1.

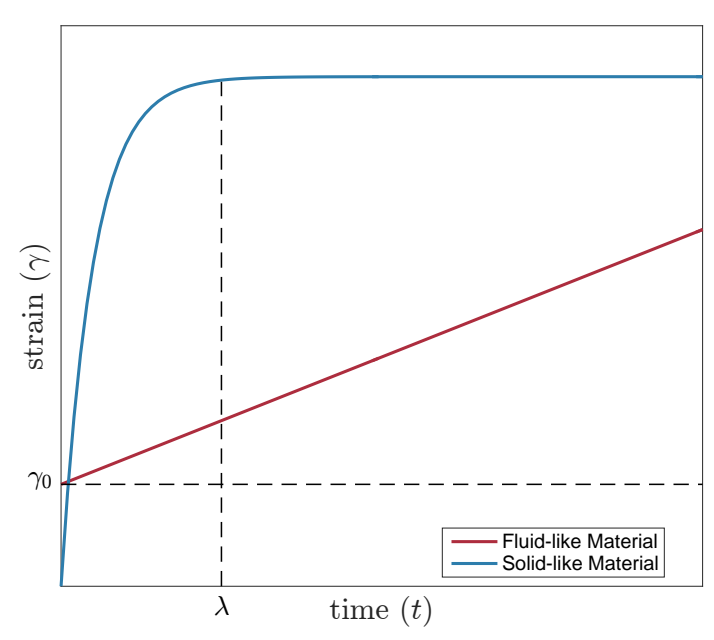

Figure A.1: Behavior of solid-like and fluid-like materials under a creep experiment

The initial deformation $\gamma_{0}$ accounts for an elastic deformation $\gamma_{e}$ caused by elastic effects on this fluid-like material. Meanwhile, the time constant $\lambda$ accounts for viscous effects on the solid-like material.

Creep experiments will be simulated in this section for both a perfect Kelvin-Voigt solid and a Maxwell liquid. 


\section{A.1.1}

\section{Kelvin-Voigt solid}

The stress imposed to the Kelvin-Voigt solid is the sum of the elastic stress $\sigma_{e}=G_{K V} \gamma$ and the viscous stress $\sigma_{v}=\eta_{K V} \dot{\gamma}$ so that

$$
\sigma=G_{K V} \gamma+\eta_{K V} \dot{\gamma} \quad \text { with } \quad \gamma=0 \text { at } \quad t=0
$$

hence

$$
\sigma=G_{K V} \gamma+\eta_{K V} \dot{\gamma} \rightarrow \sigma=G_{K V} \gamma+\eta_{K V} \frac{d \gamma}{d t}
$$

thus, rearranging the equation above

$$
\frac{G_{K V}}{\eta_{K V}} d t=\frac{d \gamma}{\left(\sigma / G_{K V}-\gamma\right)}
$$

integrating this equation with respect to time, we obtain

$$
\frac{G_{K V}}{\eta_{K V}} t=-\ln \left[\left(\sigma / G_{K V}\right)-\gamma\right]+C
$$

Applying the boundary condition results in $C=\ln \left(\sigma / G_{K V}\right)$. Hence, rearranging equation A-4 in relation to $\gamma$ we obtain

$$
\gamma(t)=\left(\sigma / G_{K V}\right)\left[1-\exp \left[\left(-G_{K V}\right) t /\left(\eta_{K V}\right)\right]\right.
$$

The retardation time $\lambda$ represents the time needed for the strain to reach $(1-1 / e)$ of its equilibrium value and is given by

$$
\lambda \equiv \frac{\eta_{K V}}{G_{K V}}
$$

Analyzing equation A-5, a plateau is expected for $\gamma(\infty)$, which clearly indicates a solid-like behavior on a large timescale as a constant stress will provoke a constant strain.

\section{A.1.1.1}

\section{Maxwell liquid}

The constitutive equation for a constant stress applied to a perfect Maxwell fluid is given by

$$
\sigma=G_{M W} \gamma_{e}=\eta_{M W} \dot{\gamma}_{v}
$$

Hence

$$
\gamma_{e}=\frac{\sigma}{G_{M W}}, \quad \frac{d \gamma_{v}}{d t}=\frac{\sigma}{\eta_{M W}}
$$

using the boundary condition $\gamma_{v}=0$ at $t=0$ and integrating the equation, we obtain

$$
\gamma_{v}=\frac{\sigma}{\eta_{M W}} t
$$


Accounting for the fact that $\gamma=\gamma_{e}+\gamma_{v}$, the strain evolution equation is given by

$$
\gamma(t)=\frac{\sigma}{G_{M W}}+\frac{\sigma}{\eta_{M W}} t \rightarrow \gamma(t)=\frac{\sigma}{G_{M W}}\left[1+\frac{G_{M W}}{\eta_{M W}} t\right]
$$

It is known that the instantaneous strain in the spring is $\gamma_{0} \equiv \sigma /\left(G_{M W}\right)$. Hence,

$$
\gamma(t)=\gamma_{0}\left[1+\frac{G_{M W}}{\eta_{M W}} t\right]
$$

and the strain evolution with time indicates a fluid-like behavior since strain increases with time for a constant stress. A limitation of the Maxwell model comes from the fact that $\eta_{M W}$ and $G_{M W}$ are constant. Hence, Newtonian flow is expected during the creep experiment, which is usually not true for generic materials.

\section{A.2}

\section{Stress relaxation}

To perform a stress relaxation experiment, a constant deformation $\gamma$ must be applied to the material at the time $t=0$. For every material, the total stress under a constant deformation will be a function of the time $(\sigma(t))$ as molecules tend to rearrange and stress is relieved. Stress will reach a plateau if the material possesses a solid-like behavior. Meanwhile, stress will continuously decrease until it reaches a neutral value of zero stress, if the material possesses a fluid-like behavior, as shown in figure A.2.

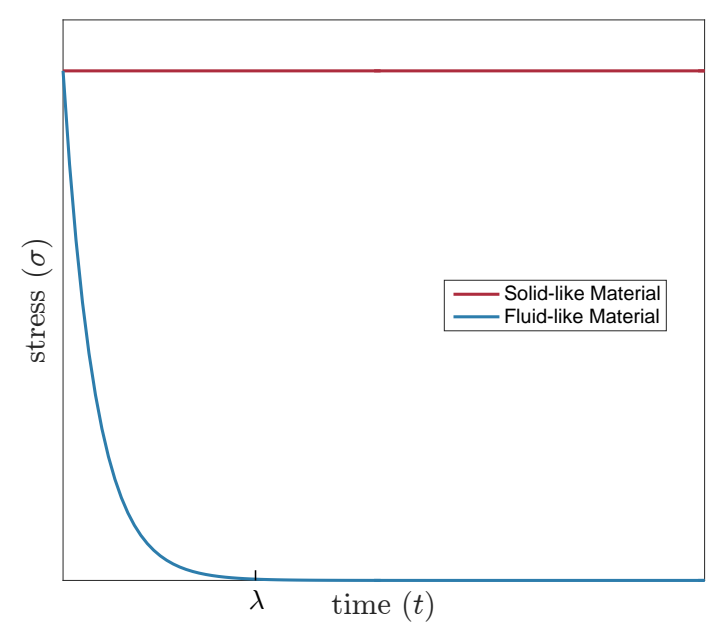

Figure A.2: Behavior of solid-like and fluid-like materials under a stress relaxation experiment 
The time constant $\lambda$ accounts for elastic effects on the fluid-like material.

Stress relaxation experiments will be simulated in this section for both a perfect Kelvin-Voigt solid and a Maxwell liquid.

\section{A.2.1}

\section{Kelvin-Voigt solid}

The Kelvin-Voigt model is not able to predict stress relaxation. Again, the mechanical analog consists of an elastic branch and a viscous branch. The elastic branch will be subjected to an elastic deformation $\gamma_{e}$ equal to the viscous deformation $\gamma_{v}$. The stress equation for the Kelvin-Voigt model is given by

$$
\sigma(t)=\sigma_{e}+\sigma_{v} \rightarrow \sigma(t)=G_{K V} \gamma+\eta_{K V} \dot{\gamma}
$$

The stress relaxation experiment imposes a constant deformation $\gamma$ to the material, but the dashpot cannot relax on this condition, since $\dot{\gamma}=0$ causing

$$
\sigma=G_{K V} \gamma
$$

which is a constant value.

\section{A.2.2}

\section{Maxwell fluid}

Once more, the constitutive equation for a constant deformation $\gamma$ applied to a Maxwell fluid is given by

$$
\sigma(t)=G_{K V} \gamma_{e}=\eta_{K V} \dot{\gamma}_{v}
$$

where $\gamma_{e}+\gamma_{v}=\gamma$.

Thus

$$
G_{K V}\left(\gamma-\gamma_{v}\right)=\eta_{K V} \dot{\gamma}_{v} \quad \text { with } \quad \gamma_{v}=0 \quad \text { at } \quad t=0
$$

hence

$$
\frac{G_{K V}}{\eta_{K V}} d t=\frac{d \gamma_{v}}{\gamma-\gamma_{v}}
$$

integrating equation $\mathrm{A}-16$ we obtain

$$
\frac{G_{K V}}{\eta_{K V}} t=-\ln \left(\gamma-\gamma_{v}\right)+C
$$

Applying the boundary condition, we obtain that $C=\ln \gamma$. Thus

$$
-\frac{G_{K V}}{\eta_{K V}} t=\ln \left[\left(\gamma-\gamma_{v}\right) / \gamma\right]
$$

taking to the exponential form 


$$
\exp \left(-\frac{G_{K V}}{\eta_{K V}} t\right)=\left(\gamma-\gamma_{v}\right) / \gamma
$$

at last

$$
\sigma(t)=G_{K V} \gamma \exp \left(-\frac{G_{K V}}{\eta_{K V}} t\right)
$$

The stress from the constant deformation experiment decays exponentially with time in the Maxwell model. The relaxation time is given by

$$
\lambda \equiv \frac{\eta_{K V}}{G_{K V}}
$$

meaning that the stress drops to $1 / e$ of its initial value at $t=\lambda$. Initially, the stress is $\sigma(0)=G_{K V} \gamma$, which is the stress on the spring element. However, it decays until eventually $\sigma(\infty)=0$.

Notice that this perspective clearly shows a fluid point of view of this phenomenon at large timescales, since it is expected that no stress is needed to sustain a constant deformation in a fluid. 
B

\section{The Jeffreys Mechanical Model}

The Jeffreys mechanical model is depicted below, in figure B.1.

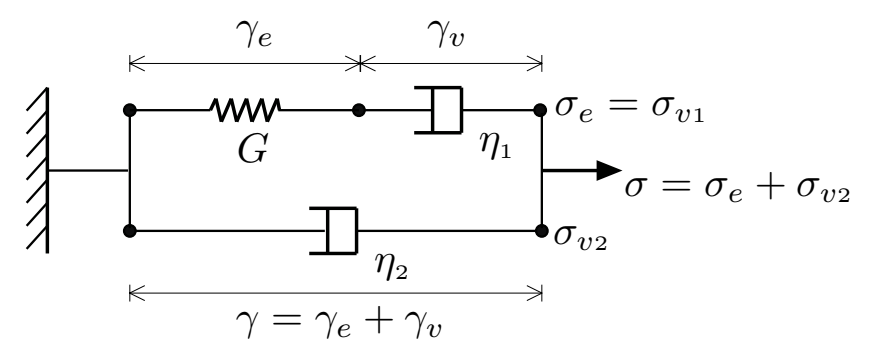

Figure B.1: The Jeffreys mechanical analog.

From figure B.1, it may be stated that this model comprises to the following equations:

$$
\begin{aligned}
\sigma=\sigma_{e}+\sigma_{v 2} & =\sigma_{v 1}+\sigma_{v 2} \\
\dot{\sigma}=\dot{\sigma}_{e}+\dot{\sigma}_{v 2} & =\dot{\sigma}_{v 1}+\dot{\sigma}_{v 2} \\
\gamma & =\gamma_{e}+\gamma_{v} \\
\dot{\gamma} & =\dot{\gamma}_{e}+\dot{\gamma}_{v}
\end{aligned}
$$

And the constitutive equations for the mechanical elements are:

$$
\begin{gathered}
\sigma_{e}=G \gamma_{e} \\
\sigma_{v 1}=\eta_{1} \dot{\gamma}_{v} \\
\sigma_{v 2}=\eta_{2} \dot{\gamma}
\end{gathered}
$$

It is important to state that

$$
\eta=\eta_{1}+\eta_{2}
$$

If we multiply equation B-4 by $\eta_{1}$

$$
\eta_{1} \dot{\gamma}=\eta_{1} \dot{\gamma}_{e}+\eta_{1} \dot{\gamma}_{v}
$$

hence 


$$
\eta_{1} \dot{\gamma}=\eta_{1} \dot{\gamma}_{e}+\sigma_{v 1}
$$

Taking the derivative of equation B-5

$$
\dot{\sigma}_{e}=\dot{\sigma}_{v 1}=G \dot{\gamma}_{e} \rightarrow \dot{\gamma}_{e}=\frac{\dot{\sigma}_{v 1}}{G}
$$

thus

$$
\eta_{1} \dot{\gamma}=\dot{\sigma}_{v 1} \frac{\eta_{1}}{G}+\sigma_{v 1}
$$

rearranging for $\sigma_{v 1}$

$$
\sigma_{v 1}=\eta_{1} \dot{\gamma}-\dot{\sigma}_{v 1} \frac{\eta_{1}}{G}
$$

Applying equation B-13 in equation B-1

$$
\sigma=\eta_{1} \dot{\gamma}-\dot{\sigma}_{v 1} \frac{\eta_{1}}{G}+\sigma_{v 2}
$$

Using equation B-7

$$
\sigma=\eta_{1} \dot{\gamma}-\dot{\sigma}_{v 1} \frac{\eta_{1}}{G}+\eta_{2} \dot{\gamma}
$$

hence

$$
\sigma=\left(\eta_{1}+\eta_{2}\right) \dot{\gamma}-\dot{\sigma}_{v 1} \frac{\eta_{1}}{G}
$$

In order to obtain $\dot{\sigma}_{v 1}$, we may apply equation B-7 in equation B-2

$$
\dot{\sigma}=\dot{\sigma}_{v 1}+\eta_{2} \ddot{\gamma} \rightarrow \dot{\sigma}_{v 1}=\dot{\sigma}-\eta_{2} \ddot{\gamma}
$$

hence

$$
\sigma=\left(\eta_{1}+\eta_{2}\right) \dot{\gamma}-\left(\dot{\sigma}-\eta_{1} \ddot{\gamma}\right) \frac{\eta_{2}}{G}
$$

rearranging

$$
\sigma+\frac{\eta_{1}}{G} \dot{\sigma}=\left(\eta_{1}+\eta_{2}\right) \dot{\gamma}+\eta_{2} \frac{\eta_{1}}{G} \ddot{\gamma}
$$

which is equation 2-75.

We may adjust equation B-19 so that $\eta$ appears in all strain-related terms

applying equation B-8

$$
\sigma+\frac{\eta_{1}}{G} \dot{\sigma}=\eta \dot{\gamma}+\eta_{2} \frac{\eta}{\eta} \frac{\eta_{1}}{G} \ddot{\gamma}
$$

$$
\sigma+\frac{\eta_{1}}{G} \dot{\sigma}=\eta \dot{\gamma}+\eta \frac{\eta_{1} \eta_{2}}{G\left(\eta_{1}+\eta_{2}\right)} \ddot{\gamma}
$$

defining the relaxation time as

$$
\theta_{1}=\frac{\eta_{1}}{G}
$$

and the retardation time as

$$
\theta_{2}=\frac{\eta_{1} \eta_{2}}{G\left(\eta_{1}+\eta_{2}\right)}
$$


we obtain

$$
\sigma+\theta_{1} \dot{\sigma}=\eta\left(\dot{\gamma}+\theta_{2} \ddot{\gamma}\right)
$$

which is equation 2-76.

Equations B-22 and B-23 may be demonstrated through creep and stress relaxation experiments.

A creep experiment is performed by inputing a constant stress to the material. Therefore, equation B-21 reduces to

$$
\sigma=\eta\left(\dot{\gamma}+\frac{\eta_{1} \eta_{2}}{G\left(\eta_{1}+\eta_{2}\right)} \ddot{\gamma}\right)
$$

since $\sigma=$ cte $\rightarrow \dot{\sigma}=0$.

hence

$$
\frac{\sigma}{\eta}=\left(\dot{\gamma}+\frac{\eta_{1} \eta_{2}}{G\left(\eta_{1}+\eta_{2}\right)} \ddot{\gamma}\right)
$$

We may state that $\ddot{\gamma}=d \dot{\gamma} / d t$. In this case

$$
\frac{\sigma}{\eta}=\left(\dot{\gamma}+\frac{\eta_{1} \eta_{2}}{G\left(\eta_{1}+\eta_{2}\right)} \frac{d \dot{\gamma}}{d t}\right)
$$

rearranging this equation, we obtain

$$
\frac{\frac{\sigma}{\eta}-\dot{\gamma}}{\frac{\eta_{1} \eta_{2}}{G\left(\eta_{1}+\eta_{2}\right)}}=\frac{d \dot{\gamma}}{d t}
$$

Separating the differential equation

$$
d t=\frac{\frac{\eta_{1} \eta_{2}}{G\left(\eta_{1}+\eta_{2}\right)}}{\frac{\sigma}{\eta}-\dot{\gamma}} d \dot{\gamma}
$$

Integrating both sides

$$
\int_{0}^{t} d t^{\prime}=\int_{\dot{\gamma}_{0}}^{\dot{\gamma}} \frac{\frac{\eta_{1} \eta_{2}}{G\left(\eta_{1}+\eta_{2}\right)}}{\frac{\sigma}{\eta}-\dot{\gamma}^{\prime}} d \dot{\gamma^{\prime}}
$$

or

$$
\int_{0}^{t} d t^{\prime}=\frac{\eta_{1} \eta_{2}}{G\left(\eta_{1}+\eta_{2}\right)} \int_{\dot{\gamma}_{0}}^{\dot{\gamma}} \frac{1}{\frac{\sigma}{\eta}-\dot{\gamma}^{\prime}} d \dot{\gamma}^{\prime}
$$

hence

$$
\frac{\eta_{1} \eta_{2}}{G\left(\eta_{1}+\eta_{2}\right)}\left(\ln \left(\frac{\sigma}{\eta}-\dot{\gamma}\right)-\ln \left(\frac{\sigma}{\eta}-\dot{\gamma}_{0}\right)\right)=t
$$

rearraging, we obtain

$$
\ln \left(\frac{\sigma}{\eta}-\dot{\gamma}\right)-\ln \left(\frac{\sigma}{\eta}-\dot{\gamma}_{0}\right)=\frac{t}{\frac{\eta_{1} \eta_{2}}{G\left(\eta_{1}+\eta_{2}\right)}}
$$

multiplying by -1 


$$
-\ln \left(\frac{\sigma}{\eta}-\dot{\gamma}\right)+\ln \left(\frac{\sigma}{\eta}-\dot{\gamma}_{0}\right)=-\frac{t}{\frac{\eta_{1} \eta_{2}}{G\left(\eta_{1}+\eta_{2}\right)}}
$$

taking the equation above to the exponential form

$$
-e^{\ln \left(\frac{\sigma}{\eta}-\dot{\gamma}\right)}+e^{\ln \left(\frac{\sigma}{\eta}-\dot{\gamma}_{0}\right)}=e^{\left.-\frac{t}{G\left(\eta_{1} \eta_{2}\right.}+\eta_{2}\right)}
$$

thus

hence

$$
-\frac{\sigma}{\eta}+\dot{\gamma}+\frac{\sigma}{\eta}-\dot{\gamma}_{0}=e^{-\frac{t}{G\left(\eta_{1}+\eta_{2}\right)}}
$$

$$
\dot{\gamma}-\dot{\gamma}_{0}=e^{-\frac{t}{\frac{\eta_{1} \eta_{2}}{G\left(\eta_{1}+\eta_{2}\right)}}}
$$

Finally,

$$
\dot{\gamma}=\dot{\gamma}_{0}+e^{-\frac{t}{\frac{\eta_{1} \eta_{2}}{G\left(\eta_{1}+\eta_{2}\right)}}}
$$

where $\eta_{1} \eta_{2} / G\left(\eta_{1}+\eta_{2}\right)$ is the creep experiment time constant. Hence

$$
\theta_{2}=\frac{\eta_{1} \eta_{2}}{G\left(\eta_{1}+\eta_{2}\right)}
$$

which is the retardation time, previously defined in equation B-23.

Analogously, a stress relaxation experiment is performed by inputting a constant deformation to the material. Therefore, equation B-21 reduces to

$$
\sigma+\frac{\eta_{1}}{G} \dot{\sigma}=0
$$

hence

$$
\sigma=-\frac{\eta_{1}}{G} \dot{\sigma}
$$

We may state that $\dot{\sigma}=d \sigma / d t$. In this case

Rearranging

$$
\sigma=-\frac{\eta_{1}}{G} \frac{d \sigma}{d t}
$$

Integrating both sides

$$
d t=-\frac{\eta_{1}}{G} \frac{d \sigma}{\sigma}
$$

hence

$$
\int_{0}^{t} d t^{\prime}=-\frac{\eta_{1}}{G} \int_{\sigma_{0}}^{\sigma} \frac{d \sigma^{\prime}}{\sigma^{\prime}}
$$

$$
-\frac{t}{\frac{\eta_{1}}{G}}=\ln \left(\frac{\sigma}{\sigma_{0}}\right)
$$

taking the equation above to the exponential form

$$
e^{-\frac{t}{\frac{\eta_{1}}{G}}}=e^{\ln \left(\frac{\sigma}{\sigma_{0}}\right)}
$$


thus

Finally, we obtain

$$
e^{-\frac{t}{\eta_{1}}}=\frac{\sigma}{\sigma_{0}}
$$

$$
\sigma=\sigma_{0} e^{-\frac{t}{\frac{\eta_{1}}{G}}}
$$

where $\eta_{1} / G$ is the stress relaxation experiment time constant. Hence

$$
\theta_{1}=\frac{\eta_{1}}{G}
$$

which is the relaxation time, previously defined in equation B-22.

The model parameters $\eta_{1}, \eta_{2}$ and $G$ and the time constants $\theta_{1}$ and $\theta_{2}$ may be described as functions of measurable quantities.

If a strain-controlled oscillatory experiment is performed, the input strain wave will have the following form

$$
\gamma=\gamma_{a} \sin (\omega t)
$$

and the shear rate will have the following form

$$
\dot{\gamma}=\dot{\gamma}_{a} \cos (\omega t)
$$

taking the derivative again

$$
\ddot{\gamma}=-\ddot{\gamma}_{a} \sin (\omega t)
$$

the output stress response wave in a constant-structure motion will be a sinusoidal wave with a phase angle

$$
\sigma=\sigma_{a} \sin (\omega t+\delta)=\sigma_{a} \sin (\omega t) \cos (\delta)+\sigma_{a} \cos (\omega t) \sin (\delta)
$$

taking the derivative

$\dot{\sigma}=\sigma_{a} \omega \cos (\omega t+\delta)=\sigma_{a} \omega \cos (\omega t) \cos (\delta)-\sigma_{a} \omega \sin (\omega t) \sin (\delta)$

Applying the wave equations above to B-24, we obtain

$$
\begin{aligned}
& \sigma_{a} \sin (\omega t) \cos (\delta)+\sigma_{a} \cos (\omega t) \sin (\delta)+\sigma_{a} \omega \theta_{1} \cos (\omega t) \cos (\delta) \\
& -\sigma_{a} \omega \theta_{1} \sin (\omega t) \sin (\delta)=\eta\left(\gamma_{a} \omega \cos (\omega t)-\theta_{2} \gamma_{a} \omega^{2} \sin (\omega t)\right)
\end{aligned}
$$

Defining the dynamic moduli as

$$
G^{\prime}(\omega)=\frac{\sigma_{a}}{\gamma_{a}} \cos (\delta), \quad G^{\prime \prime}(\omega)=\frac{\sigma_{a}}{\gamma_{a}} \sin (\delta)
$$




$$
\begin{aligned}
G^{\prime} \gamma_{a} \sin (\omega t)+ & G^{\prime \prime} \gamma_{a} \cos (\omega t)+G^{\prime} \gamma_{a} \omega \theta_{1} \cos (\omega t) \\
& -G^{\prime \prime} \gamma_{a} \omega \theta_{1} \sin (\omega t)=\eta\left(\gamma_{a} \omega \cos (\omega t)-\theta_{2} \gamma_{a} \omega^{2} \sin (\omega t)\right)
\end{aligned}
$$

We may group the sine and cosine terms

$$
\sin (\omega t)\left(G^{\prime} \gamma_{a}-G^{\prime \prime} \gamma_{a} \omega \theta_{1}+\theta_{2} \gamma_{a} \omega^{2}\right)+\cos (\omega t)\left(G^{\prime \prime} \gamma_{a}+G^{\prime} \gamma_{a} \omega \theta_{1}-\eta \gamma_{a} \omega\right)=0
$$

Notice that both sine and cosine coefficients must be equal to zero.

Hence

$$
G^{\prime \prime} \gamma_{a}+G^{\prime} \gamma_{a} \omega \theta_{1}-\eta \gamma_{a} \omega=0
$$

thus

and

$$
\theta_{1}=\frac{\eta \omega-G^{\prime \prime}}{G^{\prime} \omega}=\frac{\eta}{G^{\prime}}-\frac{G^{\prime \prime}}{G^{\prime} \omega}
$$

$$
G^{\prime} \gamma_{a}-G^{\prime \prime} \gamma_{a} \omega \theta_{1}+\theta_{2} \gamma_{a} \omega^{2}=0
$$

thus

$$
\theta_{2}=\frac{G^{\prime \prime}}{G^{\prime} \omega}-\frac{G^{\prime 2}+G^{\prime \prime 2}}{G^{\prime} \omega^{2} \eta}
$$

which are equal to equations $2-83$ and 2-84. At last, the combination between equations B-8, B-22, B-23, B-60 and B-62 generate equations 2-85, 2-86 and 2-87.

Likewise, if a stress-controlled oscillatory experiment is performed, the input stress wave will have the following form

$$
\sigma=\sigma_{a} \sin (\omega t)
$$

and the stress derivative will have the following form

$$
\dot{\sigma}=\sigma_{a} \omega \cos (\omega t)
$$

the output strain response wave in a constant-structure motion will be a sinusoidal wave with a phase angle $\phi=-\delta$, being $\delta$ the phase angle between the stress and strain waves using the strain wave as the referential, as it was done for the strain-controlled experiment.

Thus

$$
\gamma=\gamma_{a} \sin (\omega t-\delta)=\gamma_{a} \sin (\omega t) \cos (\delta)-\gamma_{a} \cos (\omega t) \sin (\delta)
$$

taking the derivative 
$\dot{\gamma}=\gamma_{a} \omega \cos (\omega t-\delta)=\gamma_{a} \omega \cos (\omega t) \cos (\delta)+\gamma_{a} \omega \sin (\omega t) \sin (\delta)$

taking the derivative again

$$
\ddot{\gamma}=\gamma_{a} \omega^{2} \cos (\omega t-\delta)=-\gamma_{a} \omega^{2} \cos (\omega t) \cos (\delta)+\gamma_{a} \omega \sin (\omega t) \sin (\delta)
$$

Applying the wave equations above to B-24, we obtain

$$
\begin{array}{r}
\sigma_{a} \sin (\omega t)+\sigma_{a} \omega \theta_{1} \cos (\omega t)=\eta\left(\gamma_{a} \omega \cos (\omega t) \cos (\delta)+\gamma_{a} \omega \sin (\omega t) \sin (\delta)\right. \\
\left.-\theta_{2} \gamma_{a} \omega^{2} \sin (\omega t) \cos (\delta)+\theta_{2} \gamma_{a} \omega^{2} \cos (\omega t) \sin (\delta)\right)
\end{array}
$$

We may group the sine and cosine terms

$$
\begin{aligned}
\sin (\omega t)\left(\sigma_{a}-\right. & \left.\eta \gamma_{a} \omega \sin (\delta)+\theta_{2} \eta \gamma_{a} \omega^{2} \cos (\delta)\right) \\
& +\cos (\omega t)\left(\sigma_{a} \omega \theta_{1}-\eta \gamma_{a} \omega \cos (\delta)-\theta_{2} \eta \gamma_{a} \omega^{2} \sin (\delta)\right)=0
\end{aligned}
$$

Defining the dynamic moduli as

$$
J^{\prime}(\omega)=\frac{\gamma_{a}}{\sigma_{a}} \cos (\delta), \quad J^{\prime \prime}(\omega)=\frac{\gamma_{a}}{\sigma_{a}} \sin (\delta)
$$

we obtain

$$
\sin (\omega t)\left(\sigma_{a}-\eta J^{\prime \prime} \omega+\theta_{2} \eta J^{\prime} \omega^{2}\right)+\cos (\omega t)\left(\sigma_{a} \omega \theta_{1}-\eta J^{\prime} \omega-\theta_{2} \eta J^{\prime \prime} \omega^{2}\right)=0
$$

Notice that both sine and cosine coefficients must be equal to zero.

Hence

$$
1-\eta \omega J^{\prime \prime}+\theta_{2} \eta \omega^{2} J^{\prime}=0
$$

thus

$$
\theta_{2}=\frac{\eta \omega J^{\prime \prime}-1}{\eta J^{\prime} \omega^{2}}
$$

and

$$
\omega \theta_{1}-\eta \omega J^{\prime}-\theta_{2} \eta \omega^{2} J^{\prime \prime}=0
$$

thus 


$$
\theta_{1}=\frac{\left(J^{\prime 2}+J^{\prime \prime 2}\right)}{J^{\prime}} \eta-\frac{J^{\prime \prime}}{J^{\prime} \omega}
$$

which are equal to equations 2-78 and 2-79. At last, the combination between equations B-8, B-22, B-23, B-75 and B-73 generate equations 2-80, 2-81 and 2-82. 


\section{C \\ Inertia Correction}

The torque needed to accelerate or decelerate the motor and geometry is given by

$$
M_{I}=I \dot{\Omega}=I \ddot{\theta}
$$

where $I$ is the moment of inertia, $\theta$ is de angular displacement and $\Omega$ is the angular velocity.

The strain $\gamma$ is related to the angular displacement by

$$
\gamma=\frac{\theta}{k_{\gamma}}
$$

where $k_{\gamma}$ is a constant factor that depends on the geometry

$$
k_{\gamma}=\left\{\begin{array}{lc}
\beta & \text { for cone-plate } \\
\frac{h}{R} & \text { for plate-plate } \\
\frac{R_{o}-R_{i}}{R_{i}} & \text { for Couette }
\end{array}\right.
$$

where $\beta$ is the cone angle, $h$ is the gap between plates, $R$ is the plate radius, and $R_{o}$ and $R_{i}$ are the outer and inner radii of the Couette geometry.

Let us analyze the case where we impose a sinusoidal strain wave

$$
\gamma=\gamma_{a} \sin (\omega t)
$$

where $\gamma_{a}$ is the strain amplitude, $\omega$ is the oscillation frequency, and $t$ is the time. In this case,

$$
\begin{array}{r}
\theta=\theta_{a} \sin (\omega t)=k_{\gamma} \gamma_{a} \sin (\omega t) \\
\dot{\theta}=k_{\gamma} \omega \gamma_{a} \cos (\omega t) \\
\ddot{\theta}=-k_{\gamma} \omega^{2} \gamma_{a} \sin (\omega t)
\end{array}
$$

Thus, the inertia torque becomes

$$
M_{I}=-I \omega^{2} k_{\gamma} \gamma_{a} \sin (\omega t)
$$

Meanwhile, the sample torque $M_{s}$ is given by 


$$
M_{s}=M_{s a} \sin (\omega t+\delta)
$$

where $\delta$ is the phase shift due to sample elasticity.

We can write $M_{s}$ in terms of the shear stress $\sigma$

$$
M_{s}=\frac{\sigma}{k_{\sigma}}
$$

where $k_{\sigma}$ is a constant factor that depends on the geometry

$$
k_{\sigma}= \begin{cases}\frac{3}{2 \pi R^{3}} & \text { for cone-plate } \\ \frac{2}{\pi R^{3}} & \text { for plate-plate } \\ \frac{1}{2 \pi R_{i}^{2} L} & \text { for Couette }\end{cases}
$$

where $L$ is the height of the Couette geometry. Note that, due to flow inhomogeneity, for the plate-plate geometry $\sigma$ stands for the apparent shear stress, i.e. the stress at the rim of an equivalent Newtonian fluid. Thus $\sigma$ needs further correction to account for flow inhomogeneity, as it was discussed in section 3.2 .

Thus,

and

$$
M_{s a}=\frac{\sigma_{a}}{k_{\sigma}}
$$

$$
M_{s}=\frac{\sigma_{a}}{k_{\sigma}} \sin (\omega t+\delta)
$$

or finally

$$
M_{s}=\frac{\sigma_{a}}{k_{\sigma}} \cos (\delta) \sin (\omega t)+\frac{\sigma_{a}}{k_{\sigma}} \sin (\delta) \cos (\omega t)
$$

And the total torque $M$ is just

$$
M=M_{s}+M_{I}
$$

It is also equal to

$$
M=M_{a} \sin \left(\omega t+\delta_{\text {raw }}\right)
$$

or

$$
M=M_{a} \cos \left(\delta_{\text {raw }}\right) \sin (\omega t)+M_{a} \sin \left(\delta_{\text {raw }}\right) \cos (\omega t)
$$

Therefore, 


$$
\begin{array}{r}
M_{a} \cos \left(\delta_{\text {raw }}\right) \sin (\omega t)+M_{a} \sin \left(\delta_{\text {raw }}\right) \cos (\omega t)= \\
\frac{\sigma_{a}}{k_{\sigma}} \cos (\delta) \sin (\omega t)+\frac{\sigma_{a}}{k_{\sigma}} \sin (\delta) \cos (\omega t)-I \omega^{2} k_{\gamma} \gamma_{a} \sin (\omega t)
\end{array}
$$

We now rearrange equation $\mathrm{C}-18$ as follows

$$
\sin \omega t\left[M_{a} \cos \left(\delta_{\text {raw }}\right)-\frac{\sigma_{a}}{k_{\sigma}} \cos (\delta)+I \omega^{2} k_{\gamma} \gamma_{a}\right]+\cos (\omega t)\left[M_{a} \sin \left(\delta_{\text {raw }}\right)-\frac{\sigma_{a}}{k_{\sigma}} \sin (\delta)\right]=0
$$

The coefficients of the above equation must be null, leading to

$$
\begin{gathered}
M_{a} \cos \left(\delta_{\text {raw }}\right)=\frac{\sigma_{a}}{k_{\sigma}} \cos (\delta)-I \omega^{2} k_{\gamma} \gamma_{a} \\
M_{a} \sin \left(\delta_{\text {raw }}\right)=\frac{\sigma_{a}}{k_{\sigma}} \sin (\delta)
\end{gathered}
$$

or

$$
\begin{gathered}
\sigma_{a} \cos (\delta)=M_{a} k_{\sigma} \cos \left(\delta_{\text {raw }}\right)+I \omega^{2} k_{\gamma} k_{\sigma} \gamma_{a} \\
\sigma_{a} \sin (\delta)=M_{a} k_{\sigma} \sin \left(\delta_{\text {raw }}\right)
\end{gathered}
$$

Thus,

$$
\sigma_{a}=\left[\left(M_{a} k_{\sigma} \cos \left(\delta_{\text {raw }}\right)+I \omega^{2} k_{\gamma} k_{\sigma} \gamma_{a}\right)^{2}+\left(M_{a} k_{\sigma} \sin \left(\delta_{\text {raw }}\right)\right)^{2}\right]^{1 / 2}
$$

and

$$
\begin{gathered}
\sin (\delta)=\frac{M_{a} k_{\sigma} \sin \left(\delta_{\text {raw }}\right)}{\sigma_{a}} \\
\cos (\delta)=\frac{M_{a} k_{\sigma} \cos \left(\delta_{\text {raw }}\right)+I \omega^{2} k_{\gamma} k_{\sigma} \gamma_{a}}{\sigma_{a}}
\end{gathered}
$$

Once we have $\sigma_{a}$ and $\delta$ we can calculate the storage and loss moduli $G^{\prime}$ and $G^{\prime \prime}$

$$
\begin{aligned}
G^{\prime} & =\frac{\sigma_{a}}{\gamma_{a}} \cos (\delta) \\
G^{\prime \prime} & =\frac{\sigma_{a}}{\gamma_{a}} \sin (\delta)
\end{aligned}
$$

